\title{
THE KINETIC THEORY OF DENSE GASES. THE CALCULATION OF THE VISCOSITY AND THERMAL CONDUCTIVITY VIRIAL COEFFICIENTS
}

\author{
Ph.D. Thesis Submitted to lowa State University, \\ February, 1973
}

M. L. Occelli
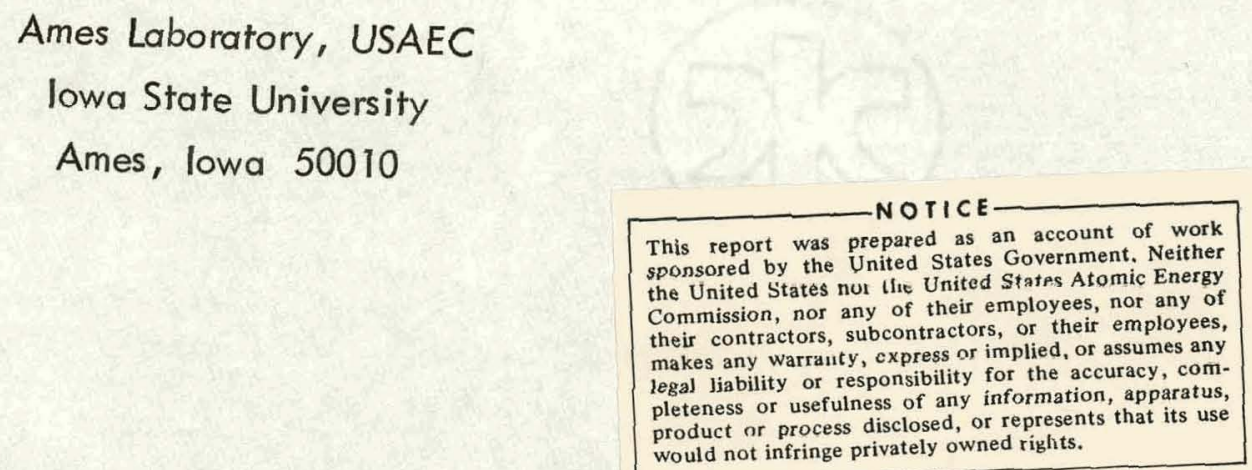

Date of Manuscript: February, 1973

PREPARED FOR THE U.S. ATOMIC ENERGY COMMISSION

DIVISION OF RESEARCH UNDER CONTRACT NO. W-7405-eng-82

\section{MASTER}




\section{DISCLAIMER}

This report was prepared as an account of work sponsored by an agency of the United States Government. Neither the United States Government nor any agency Thereof, nor any of their employees, makes any warranty, express or implied, or assumes any legal liability or responsibility for the accuracy, completeness, or usefulness of any information, apparatus, product, or process disclosed, or represents that its use would not infringe privately owned rights. Reference herein to any specific commercial product, process, or service by trade name, trademark, manufacturer, or otherwise does not necessarily constitute or imply its endorsement, recommendation, or favoring by the United States Government or any agency thereof. The views and opinions of authors expressed herein do not necessarily state or reflect those of the United States Government or any agency thereof. 


\section{DISCLAIMER}

Portions of this document may be illegible in electronic image products. Images are produced from the best available original document. 


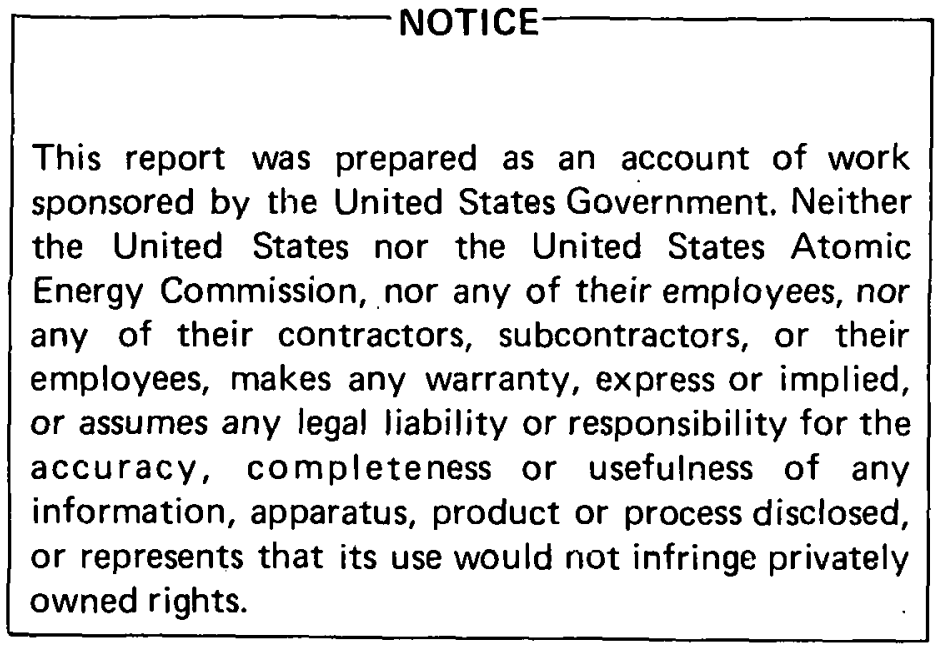

Available from: National Technical Information Service Department $A$

Springfield, VA 22151

Price: Microfiche $\$ 0.95$ 
The kinetic theory of dense gases. The calculation of the viscosity and themal conductivity virial coefficients

by

Mario Lorenzo Occelli

A Dissertation Submitted to the

Graduate Faculty in Partial Fulfillment of

The Requirements for the Degree of

DOCTOR OF PHILOSOPHY

Department: Chemistry

Major: Physical Chemistry

Approved:

D. K. Hoffman

In Charge of Major Work

For the Major Department

For the Graduate College

Iowa State University

of Science and Technology

Ames, Iowa

1973 
TABLE OF CONTENTS

ABSTRACT

1. INTRODUCTION

Page

2. SELF CONSISTENT APPROXIMATIONS IN THE KINETIC THEORY OF A MODERATELY DENSE GAS

2.1 Statistical Mechanical Theory of Transport

Phenomena

2.2 Self Consistent Approximations

2.3. Euler's Equations for an Imperfect Gas

2.4 The Correlated Velocities and the Temperature

2.5 A General Outline of a Method of Procedure to Evaluate Transport. Coefficients for a Dense Gas

40

2.6 Correlated Parameters for Hard Spheres

3. THE VISCOSITY AIND THERMAL CONDUCTIVITY SECOND VIRIAL COEFFICIENTS

3.1 The Kinetic Equation of a Moderately. Dense Gas. 49 Enskog's Equation from the BBGKY Hierarchy

3.2 The Perturbation Solution of Boltzmann's Equation 56

3.3 The Viscosity Coefficient 62

3.4 The Thermal Conductivity Coefficient $\quad 64$

4. TRANSPORT COEFFICIENTS FOR THE HARD SPHERE AND 66

SQUARE WELL MODELS

4.1 Transport Coefficients for Hard Spheres 66

4.2 The Square Well Potential Model 69

4.3 Transport Coefficients for the Square Well 75 Potential 
5. TRAINSPORT COEFFICIENTS FOR THE LENNARD-JONES MODEL 84 5.1 The Description of the Dynamics of Collision. 84

5.2 Reduction of the Virial Coefficients to Computa- 89 tional Forms

6. NUMERICAL RESULTS AND CONCLUSIONS . 98

6.1 The Square Well Calculations 98

6.2 The Lennerd-Jones Calculations 100

6.3 Numerical Results $\quad \therefore \quad 103$

7. LITERATURE CITED 124

8. ACKNOWLEDGMENTS 127

9. APPENDIX A 128

10. APPENDIX, B .. 129 
The kinetic theory of derise gases. The calculation of the viscosity and thermal conductivity virial coefficients

Mario Lorenzo Occelli

Under the supervision of $D$. K. Hoffman From the Department of Chemistry

Iowa State University of Science and Technology

The kinetic theory of moderately dense gases composed of spherically. symetrical molecules obeying a realistic potential, is developed from the BBGKY equations.

A new truncation procedure of the BBGKY hierarchy is proposed. It is shown that if the set of functions $\left(f^{(k)}\right)_{k=1}^{n}$ is replaced by the set $\left(f_{0}^{(k)}\right)_{k=1}^{n}$ of equilibrium form, the zeroth, first and trace of the second velocity moments of the equations of the hierarchy yield generalized conservation equations correct to terms linear in the macroscopic gradients. The members of the set $\left(f_{0}^{(k)}\right)_{k=1}^{n}$ are functionals of certain correlation functions which are found from the solution of differential equations obtained by taking velocity moments of the BBGKY equations. The substitutions $f^{(k)}=f_{0}^{(k)}+f_{c}^{(k)} k=1,2 \ldots n$, transform the BBGKY hierarchy into a set of coupled equations governing the unknown functions $\left(f_{c}^{(k)}\right)_{k=1}^{n}$. These can be determined once a suitable truncation of the hierarchy is made. The advantage of this procedure is the inclusion of those 
exact correlations which are necessary to derive generalized conservation equations.

A convergent form of the $n$-particles mass, momentum and energy equations are obtained by introducing the cluster expansion of the zeroth first and second velocity moments of $f_{0}^{(k)}$. For $k=2$ these moments are: the pair density $n_{0}^{(2)}$, the correlated velocities $\mathcal{U}^{(2)}$ and the temperature tensor $\underline{\tau}^{(2)}$. Once these correlations are known, $f_{0}^{(2)}$ is uniquely defined. The tensors $\mathcal{U}^{(2)}$ and $\underline{\underline{\tau}}^{(2)}$ are decomposed into their solenoidal and irrotational parts. The irrotational parts are determined by the differential equations which the tensors satisfy together with appropriate boundary conditions. However, the solenoidal parts can be arbitrarily chosen.

For hard spheres a form of $\mathrm{f}_{0}^{(z)}$ is assumed from Enskog's theory and the solenoidal and irrotational parts of $\mathcal{U}^{(2)}$ and $\underline{\tau}^{(2)}$ are evaluated. For other molecular models the solenoidal parts of $\underline{U}^{(2)}$ and $\underline{\tau}^{(2)}$ are defined in such a way that they are consistent with the results for the hard sphere gas.

It is assumed that bound states do not contribute to the collision mechanism that drives the system to equilibrium, and hence $f_{c}^{(2)}$ is taken to be zero on bound trajectories. The effect of bound states is contained in the correlations built into $f_{0}^{(2)}$. On nonbounded trajectorles $f_{C}^{(2)}$ is determined from binary collision dynamics and the molecular chaos assumption. The choice of $f_{0}^{(2)}$ is such that $f_{c}^{(2)}$ alone contributes to the 
fluxes.

WIth $f^{(2)}=f_{0}^{(2)}+f_{c}^{(2)}$ a kinetic equation for $f_{c}^{(1)}$ is obtained whioh differs from Boltzmann's equation by a corrective term containing effects of velocity and position correlations. For hard spheres, this kinetic equation reduces to Enskog's equation for the osse $\nu_{0}^{(2)}=1 ; \gamma_{0}^{(2)}$ is the oquilibrium radial distribution function.

The Ghapman and Enskog method is employed to solve the generalized Boltzmenn's équation, and different molecular models are then used to test the theory. For hard spheres transport coefficients are found to be in agreement with Enskog's to terms linear in the density. The square well and Lennard-Jones models are used to determine the effect of bound states on the transport coefficients.

Calculated virlal coeffloients are found which are in satisfactory agreement with experiments over the entire temperature range. 
1. INTRODUCTION

The purpose of the kinetic theory of gases is to explain the irreversible phenomena which are observed whenever a gas is not in a state of equilibrium.

An isolated system is said to be in equilibrium when its macroscopic parameters are constant in time. Furthermore, if. the system is not rotating and is free from external forces, the density $n$, the streaming velocity $\underline{u}$ and the temperature $\mathrm{T}$ (the macroscopic parameters) of the gas are uniform. If a gas is not in equilibrium, fluxes will form within the gas with the effect of establishing, very quickly, uniformity in the macroscopic parameters. In fact, after a time $\tau, \tau \approx 5 t_{0} \approx 10^{-9}$ seconds, ( $\tau_{0}$ is the ratio of the mean free path and some mean molecular speed $\vec{v}$ ), the irreversible phenomena cause the system to relax toward a state of local equilibrium characterized by a set of local macroscopic parameters $(n, \underline{u}, T)$ and by a velocity distribution function $\mathrm{f}^{(1)} \approx$ for ( $(\underline{\mathrm{y}}, \underline{\underline{r}, t})$ of "Maxwellian form"

$$
f_{0}^{(1)}=n(m / 2 \pi k T)^{3 / 2} e^{-(m / 2 k T)(v-u)^{2}}
$$

defined such that $f^{(1)} \mathrm{d} \underline{\underline{r}} \mathrm{~d} \underline{\mathrm{V}}$ gives the average number of molecules that can be found in a unit volume dㅍ with velocities in the range $\underline{v}, \underline{v}+\underline{v}$ during a time interval $t, t+\delta t$. with $\delta t) \tau_{0}$. In Eq. (1.1) the local macroscopic parameters are functions of position and time. The time dependence of 
$n$, u and $T$ is secular in the sense that on the $\tau_{0}$ time scale these functions are constant, their change occurring only on the macroscopic time scale. By definition

$$
\begin{array}{ll}
n=\int d \underline{v} f^{(1)}=\int d \underline{v} f_{0}^{(1)} & 1.2 \\
n \underline{u}=\int d \underline{v} \underline{v} f^{(1)}=\int d \underline{v} \underline{v} f_{0}^{(1)} & 1.3 \\
\frac{3 n k T}{2}=\int d \underline{v} \frac{m}{2}(\underline{v}-\underline{u})^{2} f^{(1)}=\int d \underline{v} \frac{m}{2}(\underline{v}-\underline{u})^{2} f_{0}^{(1)} & 1.4
\end{array}
$$

It is easy to verify that $f_{0}^{(1)}$ as defined in Eq. (1.1) satisfies these equations.

By a macroscopic parameter is meant any measurable property of the system whose value is averaged over a macroscopically small time interval $\tau_{0}$ but large enough microscopically not to be sensitive to the size of the interval chosen. An example will clarify this last statement.

A Pitot tube (1) immersed into a moving fluid will record the time averaged of the force exerted by the fluid on the surface of the tube normal to the direction of the fluid motion. If this area would be of molecular size, and the time lag of the apparatus nonexistent, a fluctuation of the measured pressure would be observed. The pressure. in this case would cease to be a macroscopic parameter in the sense a macroscopic parameter is used. Furthermore, if we let lo be the significant length of a region of space in a gas, small enough macroscopically, to be in a state of local equilibrium, then $\dot{v} \tau=l_{0}$ and $\rho_{0}>\ell$, the mean free path of a molecule. This 
will imply that if a molecule is in the region at time $t=0$, at $t=\tau_{0}$ it will still be in the region since it had not enough time to escape. Using this same argument we can reach the important conclusion that $\mathrm{n}$, $\underline{\mathrm{u}}$ and $\mathrm{T}$ are macroscopic parameters because they correspond to the average of collisionally conserved quantities. In fact, when two molecules of a dilute gas collide, their mass, momentum and kinetic energy are conserved. Therefore, the average amount of mass, momentum and kinetic energy contained in the region $\underline{d}$, during a time interval $\delta t>\tau_{0}$, but macroscopically very small, will stay unchanged after the collisions that in time $\delta t$ have occurred in dr. It takes longer than time $\tau_{0}$ for $n$, $\underline{u}$ and $T$ to change in a small region. Usually $\tau_{0}$ is of the order $10^{-9}$ seconds. The time needed by a macroscopic parameter to change, is of the order of $10^{-3}$ sec.

The above remark suggests the existence of another relaxation time $\mathrm{T}_{c} \approx \mathrm{L} / \mathrm{C}_{s} \approx 10^{-3}$ sec., $\mathrm{L}$ is a macroscopic length and $\mathrm{C}_{s}$ the sound velocity. During this stage, the hydrodynamic stage, of the relaxation of the system toward a true equilibrium state, the time evolution of the system is determined by the rate of change of $n$, $\underline{u}$ and $T$ as given by the three hydrodynamic equations. This set of equations is a direct consequence of the equations governing the conservation of mass, momentum and energy, and is:

$$
\frac{\partial}{\partial t} \rho=-\nabla \cdot \rho \underline{\underline{u}}
$$




$$
\begin{array}{lll}
\delta_{t} \rho \underline{u}=-\nabla \cdot \rho \underline{u} \underline{u}-\nabla \cdot \underline{p} & 1.6 \\
\delta_{t} \rho \tilde{U}=-\nabla \cdot \rho \underline{u} \tilde{U}-\nabla \underline{u}: \underline{\underline{p}}-\nabla \cdot \underline{q} & 1.7
\end{array}
$$

where $p=n m$ is the mass density; $\tilde{U}=\frac{u}{2}^{2}+\tilde{E}$ is the total energy per unit mass, $\tilde{E}$ is the internal energy per unit mass defined in section 2.3; 9 and $\underline{\underline{P}}$ are the heat and pressure tensors. Macroscopically the hydrodynamic equations can be interpreted as saying that in a fixed volume element mass, momentum and energy change in time because of streaming of fluid across dS, the differential element of surface area of the fixed volume, and because of the existence of gradients in $\nabla \mathrm{T}$ and $\nabla \underline{u}$. These gradients cause the fluxes of momentum $\underline{\hat{\theta}} \cdot \underline{\underline{p}}$ and thermal energy $\underline{\hat{e}} \cdot \underline{q}$ across dS given in Eqs. $(1.6,1.7)$; $\hat{\underline{\theta}}$ is a unit vector normal to dS. The last r.h.s. term of Eq. (1.7) contains the change of total energy in the volume element due to the work done by the forces $\stackrel{P}{=}$ (viscous and hydrostatic forces) acting on dS. Using the continuity equation, we can eliminate. the convective term in Eq. (1.6). In this case Eq. (1.6) reduces simply to a statement of Newton's second law for the fluid. Similarly liqs. $(1.5,1.6)$ can be used to eliminate the time derivatives of $\mathrm{n}$ and $\underline{u}$ from Eq. (1.7). In this way, we obtain an equation for $\tilde{E}$ from $E q .(1 \cdot 7)$. For an ideal fluid (a fluid for which $\stackrel{P}{=}=\mathrm{pU}, \mathrm{q}=0$ where $\mathrm{p}$ is the hydrostatic pressure) Eqs. $(1.5,1.6,1.7)$ are known as Euler's equations. These equations are discussed in section 2.3.

Finally, after a time of the order of $T_{0}$, the dissipa- 
tive fluxes will cause the gradients to disappear and the system reaches a true state of equilibrium with $f^{(1)}=f_{\text {eq. }}^{(1)}$. defined as in Eq. (1.1) but with $n=n(r), T$ and $\underline{u}_{=} \underline{u}_{0}$ t $w_{0} \times \underline{r}$ uniform everywhere and constant in time. $\underline{u}_{0}$ and $\underline{w}_{0}$ are some constant linear and angular velocities.

The fluxes $\underline{q}$ and $\underline{\underline{p}}$ (like $n, \underline{u}$ and $T$ ) are molecular averages as we can see from the definitions given in sections 3.3 and 3.4, in which $q$ and $p$ are given in terms of $f^{(1)}$ and $f^{(2)}$. However, for the dilute gas case $\underline{q}$ and $\underline{\underline{p}}$ can be calculated from $f^{(1)}$ only. In 1872 Boltzmann. (2) published his famous integrodifferential equation for $f^{(i)}$, the one particle velocity distribution function. This equation was intuitively derived using as a model a dilute gas composed of structureless molecules undergoing only binary collisions. Maxwell solved Boltzmann's equation for a gas whose molecules are point centers of force proportional to $r^{-5}$, where $r$ is the internuclear distance, but soon he realized from studies of the temperature dependence of the coefficient of viscosity, that his model was incorrect (3). Efforts to solve the Boltzmann equation for gases composed of molecules obeying more realistic potentials were in vain. Even Boltzmann had doubts on the possibility of obtaining numerical results for transport coefficients from his equation.

Chapman in 1916 and Enskog in 1917 independently solved Boltzmann's equation for a simple gas whose molecules possessed only translatory kinetic energy. Chapman and Enskog 
in Ref. (3) approached the problem in the following way: if the whole gas, is assumed to be divided into small regions microscoplcally large, then as, previously discussed, each of these regions can belassumed to be noarly in a state of local equilibrium characterized by the local temperature $T(\underline{r}, t)$, local density $n(\underline{r}, t)$ and local mean velocity $\underline{u}(\underline{r}, t)$. The distribution of velocities $f^{(1)}$ is then expressible as a functional of $n, T$ and $\underline{u}$ and given by

$$
f^{(1)}=f_{0}^{(1)}(1+\Phi)
$$

where $\Phi$ is the distortion to the local equilibrlum distribution of velocities, $f_{0}^{n}$, due to the existence of gradients in temperature, density and streaming velocity between this region and the surrounding space. It is this distortion which gives rise to the dissipative fluxes $\underline{\underline{p}}$ and $q$ appearing in the hydrodynamic equations. From Eqs. $(1.2,1.3,1.4)$ it follows that fo' $\Phi$ does not contribute to molecular averages of collisionally conserved quantities:

The basic assumption behind the Chapman and Enskog method of solution is that $f^{(1)}$ has only an inplicit time dependence through $n$, $\underline{u}$ and $T$ which can be eliminated with the help of the hydrodynamic equations derived from the conservation equations, $(1.5,1.6,1.7)$. Then Eq. (1.8) is substituted into Boltzmann's equation. By assuming that all gradients are small (a near local equilibrium state), it is possible to discard all terms nonlinear in the gradients. 
After collecting the zeroth and first order terms in $\nabla$, we obtain a set of two equations whose solutions yield $f_{0}^{(1)}$ and $f_{0}^{(1)} \Phi$, the functions neoded to evaluate the fluxes. It 1 s found that

$$
\begin{aligned}
& \eta=\eta_{0}(T) \\
& \lambda=\lambda_{0}(T)
\end{aligned}
$$

the density independent viscosity and thermal conductivity coefficients $\eta_{0}$ and $\lambda_{0}$ are defined in reference (3).

These results represented a great triumph for the kinetic theory of gases in that macroscopic laws, relating fluxes and gradients were given a microscopic basis. Moreover, predictions of transport coefficients for dilute gases were fairly well in agreement with experimental data as extensive checking of the temperature dependence of $\eta_{0}$ and $\lambda_{0}$ indicated. The fact that the above coefficients were not density dependent, had already been established by mean free path arguments. Transport coeficients represent the ability that molecules of a gas have to carry from one point to another a certain molecular property. The distance over which this transport occurs is given by $l$, the mean free path which in a dilute gas is proportional to $i / n$, while the number of carriers of molecular properties is also proportional to n. Combining the dependence of $\eta_{0}$ and $\lambda_{0}$ upon $l$ and $n$ we find an explanation for Eqs. $(1.9,1.10)$. 
It is important to realize that Chapman and Enskog's solution is not a general solution of Boltzmann's equation since Eq. (1.8) is just a particular solution (the normal solution), the one in which the time dependence is contained entirely in the functions $n(\underline{r}, t), \underline{u}(\underline{r}, t)$ and $T(\underline{r}, t)$. Howover, any solution $f(\underline{r}, \underline{v}, t)$ goos to $f(\underline{r}, \underline{v} \mid \underline{n}(\underline{r}, t), \underline{u}(\underline{r}, t)$, $T(\underline{r}, t)$ ) after a time of the order of $10^{-9}$ seconds and therefore, for many physical applications only solutions like the ones in liq. (1.8) are of practical importance.

In 1922 Enskog (3) modified Boltzmann's equation for dense gases. The Enskog theory makes use of rigid spheres. The model accounts for the energy and momentum which during rigid spheres collisions are instantaneoúsly exchanged over $\sigma$, the distance between the centers of mass of the colliding molecules. Furthermore, Enskog's theory accounts for higher order collisions in an approximate manner. It does not take into consideration the effect of molecular bound states (since these states are not allowed by the model) or rigorously account for multibody collisional effects.

Enskog's work represented an important step toward the formulation of a kinetic theory for dense gases. It was, however, limited to the particular molecular model used and to the various approximations introduced by the theory. The problem was now to find how the fluxes of molecular properties are affected by collisional transfer, multibody colli- 
sions, and bound molecular states in a gas of molecules which obey a more realistic potential.

It is at this point that the role of statistical

mechanics becomes of great importance to the development of the modern kinetic theory. 


\section{SELF CONSISTENT APPROXIMATIONS IN THE KINETIC THEORY OF A MODERATELY DENSE GAS}

2.1 Statistical Mechanical Theory of Transport Phenomena (4)

The dynamical state of a system composed of $\mathrm{N}$ molecules without internal structure, is given by. specifying $3 \mathrm{~N}$ positions and $3 \mathrm{~N}$ momenta. Such a large amount of information is not avallable and would be of limited usefulness. We are therefore limited to give a probabilistic description of the dynamical state of the system (5). This is done by a statistical examination of a large collection of $\Gamma_{0}$ macroscopically identical systems, called the ensemble. The main objective of statistical mechanics is to calculate the properties of the system under consideration. from the properties of the ensemble. In a $6 \mathrm{~N}$-dimensional phase space, the $\gamma$ space, the $\Gamma_{0}$ phase points are represented by a cloud of points whose density (number of phase points per unit phase volume) is defined by

$$
\rho^{(N)}\left(\underline{\underline{r}}^{N}, \underline{\underline{p}}^{N}\right) d \underline{\underline{x}}^{(N)}
$$

where

$$
\underline{r}^{N}=\underline{r}_{1}, \underline{r}_{2} \ldots \underline{r}_{N}, \quad \underline{p}^{N}=\underline{p}_{1}, \underline{p}_{2} \ldots \underline{p}_{N}, \quad \underline{x}^{N}=\underline{r}^{N}, \underline{p}^{N}
$$

$\underline{r_{j}}$ and $\underline{p}_{j}$ are the position and momentum vectors for particle $j$. The probability of finding a system in the unit phase volume $d \underline{X}^{N}$ is defined to be $P^{(N)}\left(\underline{\underline{r}}^{N}, \underline{p}^{N}\right) d \underline{x}^{(N)}$. The functions $P^{(N)}$ and 
$\rho^{(N)}$ are related by

$$
P^{(N)}=\rho^{(N) / \Gamma_{0}}
$$

and are normalized so that

$$
\begin{aligned}
& \int d \underline{x}^{(N)} p^{(N)}=1 \\
& \int d \underline{x}^{(N)} \rho^{(N)}=\Gamma_{0}
\end{aligned}
$$

For future convenience we introduce another probability density $\mathrm{P}^{(h)}\left(\underline{\underline{r}}^{h}, \underline{\underline{p}}^{h}\right)$ which is defined such that if we take from the $N$ molecules of a system a set of $h$ molecules, $(h)=$ $(1,2,3 \ldots h)$, then $\mathrm{P}^{(h)} \mathrm{dx}^{h}$ is the probability that molecule 1 has phase $d \underline{X}_{1}$, molecule 2 has phase $d \underline{X}_{2} \ldots$ molecule $h$ has phase $\mathrm{dX}_{h}$. The function $\mathrm{P}^{(h)}$ can be obtained directly from $P(\mathbb{N})$ by integrating $P^{(N)}$ over the phase elements of the remaining $\mathrm{N}-\mathrm{h}$ molecules

$$
P^{(h)}=\int d \underline{x}^{(N-h)} P^{(N)}
$$

and $\quad d \underline{x}^{(N-h)}=d \underline{x}_{h+1} \quad d \underline{X}_{h+2} \quad \ldots d \underline{x}_{N}$

Since in the present kinetic theory we will consider only gases composed of identical molecules, probability functions which distinguish between molecules will be of no particular interest, and we henceforth assume that $P^{(N)}$ and $P^{(h)}$ are symmetric functions.

An alternative to the representation of the state of the system by one point in $\gamma-$-space, is the representation of the system by a cloud of points in a 6-dimensional phase 
space, the $\mu$-space. A given point determines the position and momentum of one molecule. If the $N$ molecules are indistinguishable then the arrangement of molecules in $\mu$-space is unaffected by particle interchange. Therefore, there are NI phase points in $\gamma$-space which correspond to the same representation in $\mu$-space. The probability $f^{(N)}\left(\underline{r}^{N}, \underline{p}^{N}\right)$ of finding a molecule (without regard to labelling) in $d \underline{x}_{1}$; a molecule in $\mathrm{dX}_{2}$, etc.; must be $\mathrm{N}$ ! greater than $\mathrm{P}^{(\mathrm{N})}$, the probability of finding a particular molecule (say molecule 1) in $d \underline{X}_{1}$, molecule 2 in $\mathrm{dx}_{2}$, etc. It follows that

$$
f^{(N)}\left(\underline{\underline{r}}^{N}, \underline{p}^{N}\right)=N 1 p^{(N)}
$$

Similarly a set of $h$ molecules can be chosen from $N$ molecules in $N ! /(N-h) !$ ways, which implies that the probability of finding the olements $d \underline{X}_{1}, d \underline{X}_{2} \ldots \underline{d X}_{h}$ occupied by any $h$ molecules can be written as

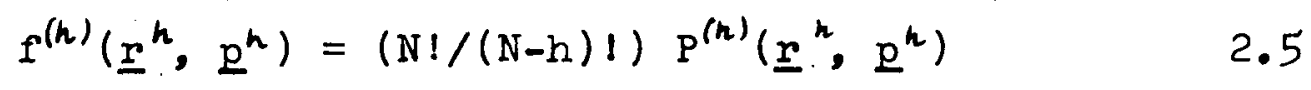

Using Eqs. $(2.3,2.4)$, the relationships between $\mathrm{P}^{(N)}$ and $\mathrm{P}^{(h)}$ $f^{(N)}$ and $P^{(N)}$, we can show that $f^{(h)}$ is related to $f^{(N)}$ as follows

$$
f^{(n)}=(1 /(N-h) 1) \int d \underline{X}^{(N-h)} f^{(N)}: 2.6
$$

This last identity will be of great importance in establishing a link between statistical mechanics and the theory of transport phenomena. 
The change in time of $f^{(N)}$ is governed by Liouville's equation, which is a continuity equation of phase points in the $\gamma$-phase space

$$
D^{(N)} f^{(N)}=0
$$

The operator $D^{(N)}$ is defined by

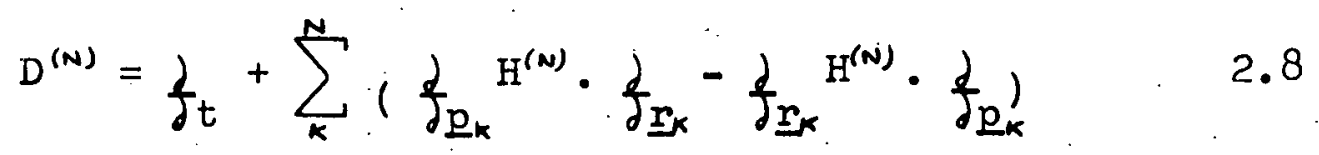

where $H^{(N)}$ is the Hamiltonian of the $\mathbb{N}$ particles. If we assume pairwise additive interactions between particles, then $\mathrm{H}^{(\mathrm{N})}$ is given by

$$
H^{(N)}=\sum_{k}^{N}\left(p_{k}^{2} / 2 m+\sum_{J<k}^{N} \phi_{k J}\right)
$$

where $\Phi_{k \cdot j}$ is the intermolecular potential between particle $k$ and $j$.

Actually we are not interested directly in the time evolution of $f^{(N)}$ since $f^{(N)}$ in general, contains much unnecessary information. What is of particular importance are the equations gnverning the time evolution of lower order distribution functions, particularly $f^{(2)}$ and $f^{(1)}$, the functions necessary for a rigorous calculation of the fluxes. of the early attempts to derive Boltzmann's equation from Liouvilie's equation, of particular interest is the work of Kirkwood (1)(6), Born and H.S. Green (7). The derivations of these authors were based on Boltzmann's "Stosszahlansatz" (2) which is equivalent to Jean's "molecular chaos assumption" 
(8). In order to introduce into the kinetic theory the effects of multiple collisions, Bogoliubov (9) replaced the Ansatz with more general assumptions. His ideas are discussed in a book by Uhlenbeck and Ford (10), and a complete list of the attempts to derive Boltzmann's equation from Liouvilie's equation can be found in review articles by Ernst, Haines, Dorfman (11), and by Cohen (12). Most of these theories rely on the formally exact BBGKY hierarchy of equations as their starting point. We now discuss these equations.

If Liouville's equation is multiplied by $1 /(\mathrm{N}-\mathrm{h}) 1$ and then integrated over the phase of the $(N-h)$ molecules, the use of the relationship between $f^{(N)}$ and $f^{(h)}$ in Eq. (2.6) leads to a hierarchy of $h$ inter-related integrodifferential equations first derived by Bogoliubov, Born, Green, Kirkwood, Yvon (13) and called BBGKY equations. For $\mathrm{h}=1,2$ we obtain

$$
\begin{array}{ll}
D^{(2)} f^{(2)}-\int d \underline{x}_{3}\left(0,3+O_{23}\right) f^{(3)}=0 & 2.10 \\
D^{(1)} f^{(1)}-\int d \underline{x}_{2} O_{12} f^{(2)}=0 & 2.11
\end{array}
$$

The interaction operator $0_{i j}$, is defined by

$$
0_{i j}=\frac{1}{m} \frac{\partial}{\partial \underline{\underline{r}}_{i}} \phi_{i} \cdot \cdot \frac{\partial}{\partial \underline{v}_{i}}
$$

Examination of the BBGKY equations suggests a procedure to obtain $f^{(2)}$ and $f^{(1)}$. After truncation of the hierarchy by finding a "suitable approximation" for $f^{(3)}$, we could solve 
Eq. (2.10) for $f^{(2)}$ and use this solution to find $f^{(1)}$ from Eq. (2.11). Furthermore from the first BBGKY equation one should be able to derive a kinetic equation which in the low density limit reduces to the Boltzmenn equation. To derive kinetic equations like Boltzmann's is a fundamental problem of tho kinotic theory of gases.

Choh and Uhlenbeck (14) used Eqs. $(2.10,2.11)$ and Bogoliubor's ideas to derive a kinetic equation containing corrections due to multibody collisions, they specifically evaluated the three body collision term. From their kinetic equation, they concluded that transport coefficients can be written as expansions in powers of the density. Dorfman and Cohen (15), Frieman and Goldman (16), and Kawasaki and Oppenheim (17), have shown that the density expansion of $f^{(2)}$ on which the Choh-Uhlenbeck kinetic equation is based, diverges after the first two terms and that therefore the density expansion for the transport coefficients proposed by Choh and Uhlenbeck, does not exist. Hanley and co-workers (18) have presented experimental evidence that the expressions for transport coefficients contain the logarithmic dependence on the density suggested by the above authors. H.S. Green neglected multibody collisions and assumed the potential to be purely repulsive to use the molecular chaos assumption to derive a kinetic equation like Boltzmann's with a corrective term due to the size of the molecules. 
Snider and Curtiss (19)(20) used Green's equation to calculate density corrections to $\eta$ and $\lambda$. Hoffman and Curtiss (21) (22) (23) refined the Snider and Curtiss results by taking implicitly into account the effect of three body collisions on the kinetic equation. They derived an equation similar to that of Green's by applying a generalization of the molecular chaos assumption to $f^{(3)}$ and then solving the first two BBGKY equations for the equilibrium case. They used for $f_{0}^{(2)}, f_{0}^{(2)}=f_{01}^{\prime(1)} f_{02}^{\prime(1)} Y_{(0)}$ where $Y_{(0)}=y e^{+\phi_{i j} / k T}, y$ is the equilibrium radial distribution function, and $f_{01}^{\prime \prime \prime}$, $f_{o 2}^{\prime \prime \prime \prime}$ are functions of $\underline{v}_{1}^{\prime}$ and $\underline{v}_{2}^{\prime}$ the pre-collision velocities of the col-. liding molecules. For the nonequilibrium case they let

$$
f^{(2)}=f_{1}^{\prime(1)} f_{2}^{\prime(1)} Y_{(0)}
$$

which is equivalent to the approximation used by Enskog in his kinetic theory of hard spheres: By retaining only the first two terms in the density expansion of $y$, three body effects were introduced into the kinetic equation.

Comparison of the temperature dependence of the density corrections to $\eta$ and $\lambda$ with experiment reveals that kinetic theories which are based on a purely repulsive potential fail completely in the low temperature regions. The purpose of the present dissertation is to derive a kinetic theory for a moderately dense gas whose spherically symmetrical molecules obey a realistic intermolecular potential which allows molecular bound states. 


\subsection{Self Consistent Approximations}

The kinetic theory of dilute gases is on a firm theoretical basis. Boltzmann's equation gives the time evolution of the state of the system, and the H-theorem proves the irreversibility of this kinetic equation and the uniqueness of $f_{e q .}^{\prime \prime \prime}$, its equilibrium solution. Chapman and Enskog solved Boltzmann's kinetic equation and found a solution, (the normal solution) $f^{(i)}=f_{0}^{(1)}+f_{c}^{(1)}$ subject to the condition that $f_{c}^{\prime \prime \prime}$ does not contribute to the zeroth, first and trace of the second velocity moments of $f^{(1)}$. In the theory, the relaxation of the system to equilibrium is given by the equations of change which relate the rate of change of the macroscopic parameters to the fluxes. These equations are obtained by taking the zeroth, first and trace of the second velocity moments of the kinetic equation. Mathematically they represent Hilbert's conditions of integrability, which are the conditions that an integrodifferential equation like the linearized Boltzmann's equation must satisfy to have a solution, (24). The non-equilibrium part of $f^{(i)}$ does not contribute to the se moments.

In this section we will extend this formalism to the kinotio theory of dense gases by introducing approximations to $f^{(1)}, f^{(2)}$ and $f^{(3)}$ consistent with the macroscopic conservation equations and capable of producing a Boltzmann equation not limited to any particular molecular model. This approach 
was first initiated by Green and Hof fman (25), and further discussed by Green (26). In this chapter we will refer extensively to their. work.

The first three velocity moments of the nth BBGKY equations are

$$
\int \prod_{c} d \underline{\underline{v}}_{t}\left(\Psi_{L}^{(n)}\right)\left[D^{(n)} f^{(N)}-\sum_{i, j}^{n} O_{i j} f^{(n)}-\sum_{k}^{n} \int d \underline{x}_{n+1} O_{k, n+1} f^{(n+1)}\right]=0
$$

and $\Psi^{(n)}=1, \Psi_{2}^{(n)}=\sum_{l}^{n} \underline{v}_{L}, \Psi_{3}^{(n)}=\sum_{l}^{n}\left(\frac{1}{2} v_{L}^{2}+\frac{1}{2} \sum_{m j}^{n} \varphi_{i j}\right)$

from which we obtain exact conservation equations for a cluster of n-particles. These are:

$\frac{\partial}{\partial t} n^{(n)}+\sum_{c} \cdot \frac{\partial}{\partial \underline{r}_{c}} n^{(n)}\left\langle\underline{v}_{c}\right\rangle^{(n)}=0$

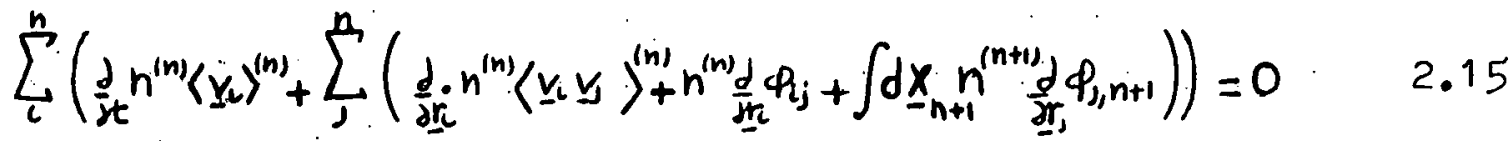

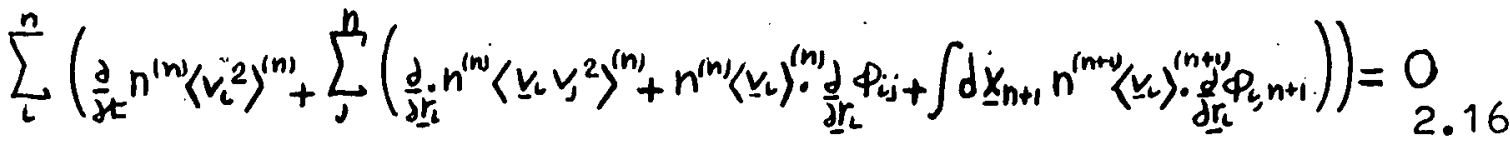
where $n^{(n)}\langle()\rangle^{(n)}=\int \prod_{L}^{n} d \underline{L}() f^{(n)}$ and $n^{(n)}$ is the n-particle density. We postulate the existence of a set of nondissipative functions $\left(f_{0}^{(n)}\right)=\left(f_{0}^{(1)}, f_{0}^{(2)} \ldots f_{0}^{(n)}\right)$ of equilibrium form which satisfy

$$
\int \prod_{i}^{n} d \underline{v}_{i i} d \underline{r}_{i i}\left(\Psi_{k}^{(n)}\right)_{k=1}^{3}\left(1 . h . \dot{s} \cdot n^{(h B B G K Y} E_{.}\right)=0 \quad 2.17
$$

to terms linear in the macroscopic gradients; $\underline{V}_{i i}$ and $\underline{\underline{r}}_{i i}$ are the relative velocity and position vectors of particles 1 and $i$. When $n=1$, the result of the substitution of $\left(f_{0}^{(n)}\right)_{n=1}^{2}$ is a 
set of differential equations free of dissipative terms known as Euler's equations, (to be discussed in section 2.3). It follows that the difference

$$
f_{c}^{(n)}=f^{(n)}-f_{0}^{(n)}
$$

must contribute only to the $\nabla^{n}, n \geqslant 2$ terms of Eq. (2.17). Therefore the functions $\left(f_{c}^{(h)}\right)$ must contain the dissipative contributions of $\left(f^{(n)}\right)$ to the fluxes. That is, for $n=1,2$

$$
\begin{aligned}
& \underline{q}=\underline{q}_{k}\left(f_{0}^{(1)}\right)+\underline{q}_{\phi}\left(f_{0}^{(q)}\right)=0 \\
& \underline{\underline{P}}=\underline{P}_{k}\left(f_{0}^{(1)}\right)+\underline{\underline{P}}_{\phi}\left(f_{0}^{(2)}\right)=p \underline{\underline{U}}
\end{aligned}
$$

and hence ( $K$ 'is' the coefficient of bulk viscosity)

$$
\begin{aligned}
& \underline{\underline{q}}=\underline{\underline{q}}_{k}\left(f_{c}^{(1)}\right)+\underline{\underline{q}}_{\varphi}\left(f_{c}^{(2)}\right)=\lambda \nabla T \\
& \underline{\underline{p}}=\underline{\underline{P}}_{k}\left(f_{c}^{(1)}\right)+\underline{\underline{P}}_{\varphi}\left(f_{c}^{(2)}\right)=2 \eta \underline{\underline{S}}+k \nabla \cdot \underline{\underline{u}} \underline{\underline{U}}
\end{aligned}
$$

$\underline{q}_{K}$, $\underline{\underline{P}}_{k}$ and ${\underline{q_{\varphi}}}_{\varphi} \underline{\underline{P}}_{\varphi}$ are the kinetic and collisional contributions to the fluxes; $\underline{\underline{S}}$, are the shear stress and unit tensors.

In Eq. (2.17) the integrations over the velocity coordinates can be done immediately, the results are a set of equations similar lo $(2.14,2.15,2.16)$. In Éqo. (2.14, 1.15) we note the existence of $\left\langle\underline{v}_{0}\right\rangle^{(n)}$ and $\left\langle\underline{v}_{c} v_{j}^{2}\right\rangle^{(n)}$ two unknown functions which can be found in principle directly from the equations in which they appear, once $\mathrm{n}^{(n)}$ is known. Therefore, one way to satisfy the first two equations for $k=1,2$ in (2.17) is to perform the integrations over the $\left(\underline{v}_{t}\right)$ and then solve for the first and second velocity moments of $f_{0}^{(n)}$. Because $f_{0}^{(n)}$ is of 
equilibrium form, all the odd velocity moments of this function will vanish and $\left\langle\underline{v}_{c} v^{2}\right\rangle^{(n)}$ will not introduce a new velocity moment into Eq. $(2.17), k=3$. For this reason the procedure just outlined above, cannot be used to prove the consistency of the energy equation in (2.17).

'In general the consistency of the Eqs. in (2.17) can be proved by comparing them with $(2.14,2.15,2.16)$, the exact equations of change and then by showing that the two sets of equations agree to terms linear in the macroscopic gradients. With this procedure the integrations over the relative coordinates must be performed, and the convergence of the integrals in Eqs. $(2.15,2.16)$ must be examined. What we propose to do is to replace the integrals in Eqs. (2.17) by a convergent set of integrals. To do this we substitute the functions $n_{0}^{(n)},\left\langle\underline{v}_{i}\right\rangle^{(n)}$ and $\left\langle v_{c}^{2}\right\rangle^{(n)}$ with some other functions which go to zero quickly as $\underline{r}_{1 i}$, $i \geq 2$, goes to $\infty$. With this purpose in mind we introduce the cluster expansion

$$
\ln n^{(a)}=\sum_{i}^{q} \sum_{p_{i}^{(1,9)}} \ln \nu^{(i)}
$$

The symbol $\sum_{\text {Ri, }}$ indicates the sum ovor all possible ways a cluster of $i$ molecules can be chosen from the set of $(q)=$ $(j, j+1 \ldots q)$ molecules. It follows that

$$
\begin{aligned}
\ln \nu^{(1)} & =\ln n^{(1)} \\
\ln \nu^{(2)} & =\ln n^{(2)}-\ln n_{1}^{(1)}-\ln n_{2}^{(1)} \\
\vdots & \nu^{(4)}=\sum_{j=1}^{9}(-1)^{(4-j)} \sum_{\text {(1),9) }} \ln n^{(j)}
\end{aligned}
$$


and $\ln \nu^{(9)} \rightarrow 0$ as any of the molecules in the q cluster is removed. Taking the time derivative of Eq. (2.22) and employing Eq. (2.14) we obtain

$$
\begin{aligned}
& \frac{\partial}{\partial t} \ln \nu^{(q)}+\sum_{j=1}^{q}(-1)^{(q-j)} \sum_{p^{(1, q)}} \sum_{i}^{j} \underline{\varphi}_{l}^{(q, j)} \cdot \delta_{\underline{r}_{L}} \ln \nu^{(j)}+\sum_{i}^{q}(-1)^{(q-1)} \partial_{\underline{r}_{L}} \cdot \underline{\varphi}_{L}^{(q) 1)}=0 \\
& \underline{\mathscr{U}}_{L}^{(n, k)}=\sum_{m=0}^{n=k}(-1)^{(m)} \sum_{p_{m}^{(k+1, n)}} \underline{u}_{\zeta}^{(m+k)} \quad \begin{array}{ll}
2.23 \\
2.24
\end{array}
\end{aligned}
$$

where by definition

$$
n^{(9)} \underline{u}_{L}^{(9)}=\int\left(\prod_{k}^{q} d \underline{v}_{k}\right) \underline{v}_{L} f^{(9)}
$$

$\underline{u}_{L}^{(9)}$ will be referred to as the correlated velocity. Eq. (2.24) is exact since it has been obtained from the exact BBGKY equations and, therefore, must be linearized before being compared with Eqs. (2.17), $k=1$. As previously mentioned, Eqs. (2.17), $n=1$ yields Euler equations for the rate of change of the macroscopic parameters of the gas at $\underline{r}_{1}$, the point under consideration. However, the correlation functions in (2.17) are nonlocal in the sense that they differ from zero when a molecule is removed from the cluster. The need to expand these functions about $\underline{r}_{1}$, suggest the following change of variables

$$
\begin{array}{ll}
\left(\underline{r}_{1}, \underline{r}_{2} \cdots \underline{r}_{n}\right) \rightarrow\left(\underline{r}_{1}, \underline{r}_{12} \cdots \underline{r}_{1 n}\right) & 2.26 \\
\left(\underline{v}_{1}, \underline{v}_{2} \ldots \underline{v}_{n}\right) \rightarrow\left(\underline{v}_{1}, \underline{v}_{12} \ldots \underline{v}_{1 n}\right) & \\
\frac{\partial}{\partial}=\left(\frac{\partial}{\partial \underline{r}_{1}}-\sum_{k}^{n} \frac{\partial}{\partial \underline{r}_{1 k}} \delta_{i 1}+\left(1-\delta_{i 1}\right) \frac{\partial}{\partial \underline{r}_{1 i}}\right. & 2.27 \\
\sum_{i}^{n} \underline{v}_{i} \cdot \frac{\partial}{\partial \underline{r}_{i}}=\underline{v}_{1} \cdot \frac{\partial}{\partial \underline{\underline{r}}}+\sum_{k=2}^{n} \underline{v}_{k} \cdot \frac{\partial}{\partial \underline{r}_{i k}} & 2.28
\end{array}
$$


$\delta_{i 1}$ is the Kronecker delta. In the spirit of the expansion employed by Chapman and Enskog, we write

$$
\begin{aligned}
& \ln \nu^{(9)}=\ln \nu_{0}^{(9)}+\varepsilon \ln \nu_{(0)}^{(q)}+\ldots . \\
& \underline{E}_{L}^{(n, k)}=\underline{\varphi}_{L(0 ;}^{(n k !)}+\varepsilon \underline{\Psi}_{L(1)}^{(n, k)}+\ldots \cdot \\
& \frac{\partial}{\partial t}=\varepsilon\left(\frac{\partial}{\partial t}\right)_{1}+\varepsilon^{2}\left(\frac{\partial}{\partial t}\right)_{2}+\ldots . \\
& \frac{\partial}{\partial \underline{r}_{1}}=\varepsilon \frac{\partial}{\partial \underline{r}_{1}}=\varepsilon \nabla
\end{aligned}
$$

where $\epsilon$. marks the order of a given term in the macroscopic gradients. All correlations in E'qs. (2.17) are assumed to be slow varying functions of macroscopic position, for this reason we retain only terms linear in $\left(\partial / \partial \underline{r}_{1}\right) \underline{r}_{12} \ldots \underline{r}_{\text {in }}$ in the theory. The correlated velocities $\mathscr{E}_{(10)}^{(n, k)}$ and $\underline{\mathscr{C}}_{(1,1)}^{(n, k)}$ are obtained from Eq. (2.24) by replacing $\underline{u}_{l}^{(m+k)}$ with $\underline{u}_{1}+\epsilon\left(\underline{u}_{l}^{(m+k)}-\underline{u}_{l}\right)$. The identities

$$
\begin{aligned}
& \underline{\varphi}_{((0)}^{(n, k)}=\underline{\mathscr{E}}_{L(0)}^{(n, K)} \delta_{n, K}=\underline{u}_{1} \delta_{n, k} \\
& \underline{\mathscr{C}}_{(1)}^{(n, k)}-\underline{\mathscr{C}}_{1}^{(n, k)}=\varepsilon\left(\underline{\varphi}_{L}^{(n, k)}-\underline{\varphi}_{1}^{(n, k)}\right)+\varepsilon^{2} \ldots \ldots
\end{aligned}
$$

follow by definition. The expansion of $\frac{\partial}{\partial t}$ is implied by the equations of change for the singlet velocity distributions functions. The set of functions ( In $\nu_{0}^{(n)}$ ) are of equilibrium form and depend, like ( $\left.\ln \nu^{(n)}\right)$, on the relative position coordinates $\left(r_{i}\right)_{i \neq 2}^{n}$. They also have a time and position dependence through 'I' $\left(\underline{r}_{1}\right)$ the temperature at the macroscopic position $\underline{r}_{1}$ The equations in (2.29) imply the following linearized con- 
23

tenuity equation

$$
\begin{aligned}
& \left(\frac{d}{\partial t}+\mathscr{E}_{1(0)}^{(q, 9)} \cdot \nabla\right) \ln V_{0}^{(q)}+(-1)^{(q-1)} \nabla_{0} \cdot \underline{E}_{1(0)}^{(q, 1)}+\sum_{j}^{q}(-1)^{(q-j)} \sum_{p_{j}(1, q)} \sum_{\dot{k}}^{q} \\
& \left(\mathscr{E}_{k}^{(q, j)}-\mathscr{E}_{1}^{(q, \mu)}\right) \cdot \frac{\partial}{\partial \underline{L}_{1 k}} \ln \nu_{0}^{(0)}+(-1)^{(q-j)} \sum_{k}^{q} \frac{\partial}{\partial \underline{r}_{1 k}} \cdot\left(\mathscr{E}_{k}^{(q, 1)} \mathscr{E}_{1}^{(q, 1)}\right)=0
\end{aligned}
$$

2.30

Since $\frac{\partial}{\partial t} \ln \mathcal{V}_{0}^{(a)}$ is known and $\mathscr{E}_{c}^{(n, m)}$ is related to $\mathscr{E}_{l}^{(n, 1)}$ by

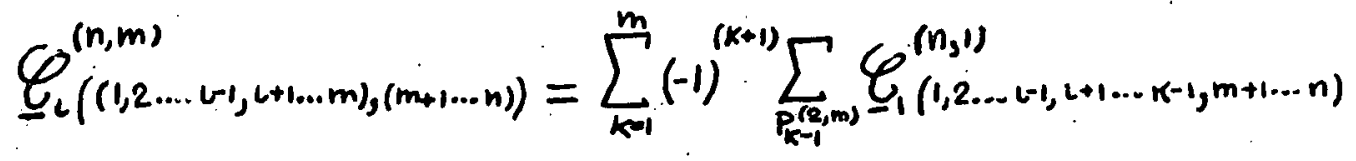

where $\underline{\varphi}_{1}^{(n, 1)}=\sum_{m=0}^{n} \sum_{p_{m}^{(2, n)}}(-1)^{m} \cdot \underline{u}_{1}^{(m+1)}$

we can say that, Eq. (2.30) is the equation the $\mathscr{\mathscr { C }}_{1}^{(n, 1)}$ must

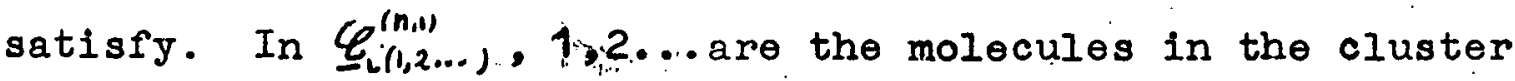
considered. As an example of the notation we give the values of $\underline{E}_{i}^{(m, s)}, m=2,3,4$

$$
\begin{aligned}
& \dot{G}_{1(2345)}^{(5,2)}=\underline{\mathscr{E}}_{1(345)}^{(4,1)}-\underline{\mathscr{E}}_{1(3452)}^{(5,1)} \\
& \underline{\mathscr{C}}_{1(2345)}^{(5,3)}=\underline{\mathscr{C}}_{1(45)}^{(3,1)}-\underline{\mathscr{C}}_{1(452)}^{(4,1)}-\underline{\mathscr{\varphi}}_{1(453)}^{(4,1)}+\underline{\mathscr{C}}_{1(4532)}^{(5,1)}
\end{aligned}
$$

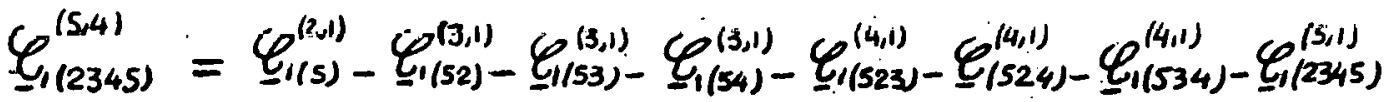

For $q=2$, Eq. (2.30) transforms to. 


$$
\left(\frac{\partial}{\partial t}+\underline{u}_{1} \cdot \nabla\right) \ln \nu_{0}^{(2)}+\frac{\partial}{\partial r} \cdot\left(\underline{u}_{2}^{(2)}-\underline{u}_{1}^{(2)}\right)-\left(\underline{u}_{2}(2)-\underline{u}_{1}^{(2)}\right)_{0} \frac{\partial}{\partial \underline{r}} \ln \nu_{0}^{(2)}-\nabla \cdot \underline{u}_{1}=0
$$

which is the equation that in section 2.4, we will solve for. $\left(\underline{u}_{2}^{(2)}-\underline{u}_{1}^{(2)}\right)$ and that in section 3.3, will be used to find the effects of the correlated velocities on the fluxes. Equation (2.32) is equivalent to E'q. (2.17) $\mathrm{k}=1, \mathrm{n}=1$.

Next, we consider a convergent form of the momentum equation integrated over the relative coordinates. By taking the time derivative of $\mathscr{C}_{q}^{(n, 1)}$ as given by Eq. (2.31) and by employing Eqs. $(2.14,2.15)$ we obtain

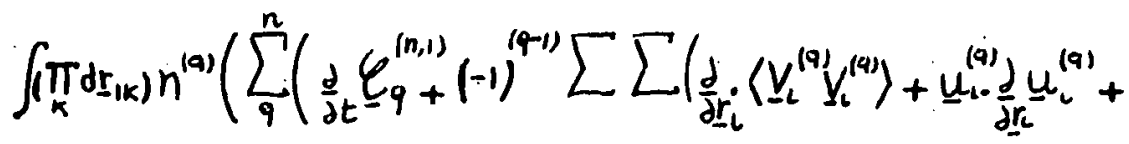

$$
\begin{aligned}
& \left.\left.\left.\left\langle V_{+}^{(q)} \underline{V}_{L}^{(9)}\right\rangle \cdot \frac{\partial}{\partial \underline{I}_{L}} \ln n^{(9)}+\underline{M}_{L}^{(9+1)}\right)\right)\right)=0
\end{aligned}
$$

where $\underline{M}_{L}^{(q+1)}=\int d \underline{\underline{r}}_{q+1} n^{(q+1)}{\stackrel{\partial}{\partial} \underline{\underline{r}}_{l}}_{l, q+1}$

$$
\underline{V}_{L}^{(9)}=\underline{v}_{L}-\underline{u}_{L}^{(9)}
$$

It is at this point that the need and motivation behind the integrations over the relative coordinates in Eqs. (2.17) becomes apparent as we will see from the argument used to replace $\mathrm{n}^{(q+1)}$ with $\mathrm{n}_{0}^{(q+1)}$ in $\mathrm{Eq} .(2.33)$.

We want to show that if $\mathrm{n}^{(9+1)}$ is expanded in a Taylor series about $\underline{r}_{1}$, because of symmetry considerations, only a 
term linear in $\nabla$ will remain in Eq. (2.34). Furthermore, if we neglect $\nabla^{n}$ terms, $n \geq 2, \underline{M}_{L}^{(q+1)}$ will be replaced by $\underline{M}_{(10)}^{(q+1)}$ which is Eq. (2.34) with $n_{0}^{(8+1)}$ instead of $n^{(4+1)}$. When $i=1$, the proof is relatively simple, therefore, we will consider the case $i \neq 1$. By definition $n^{(q+1)}$ is a totally symmetrical function with respect to particle interchange implying that

$$
\begin{aligned}
& \int \prod_{K} d \underline{\underline{r}}_{1 K} \underline{\underline{M}}_{L}^{(q+1)}=\underline{\underline{M}}_{(1) i}^{(q+1)}+\underline{\underline{M}}_{(2) i}^{(q+1)} \\
& \underline{M}_{(1) i}^{(q+1)}=\frac{1}{2} \int \prod_{k} d \underline{r}_{1 x} \int d \underline{r}_{q+1} n^{(q+1)}\left(\underline{x}^{(q+1)} \underline{r}_{i 2} \cdot \underline{r}_{\ln }\right){ }_{\partial \underline{r}_{1}} \underline{\varphi}_{1, q+1} \\
& \underline{M}_{(2) i}^{(a+1)}=\frac{1}{2} \int \prod_{k} d \underline{r}_{1 k} \int d \underline{r}_{q+1} n^{(q+1)}\left(\underline{x}^{(q+1)} \underline{r}_{n+1,2} \cdot \underline{r}_{n+1, i}\right){ }_{\partial}^{\partial} \phi_{\underline{r}_{q+1}} \quad \cdot \quad 2.37
\end{aligned}
$$

where $\underline{x}^{(q+1)}$ is the center of mass of $q+1$ molecules. In $M_{(2) i}^{(q+1)}$ change the variables of integrations to $\left(\underline{r}_{q+i, i}\right)_{l=1}^{n}$, by using the relationship

$$
\underline{\underline{r}}_{9+1, i}=\underline{r}_{i i}+\underline{r}_{4+1,1}
$$

The Jacobian of the transformation is unity. In fact

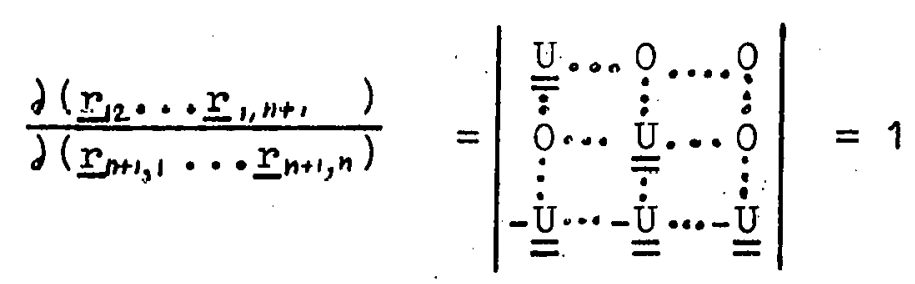

If we substitute

$$
\underline{x}^{(q+1)}=\underline{r}_{1}-\frac{q}{q+1} \underline{r}_{n+1,1,}+\frac{1}{n+1} \sum_{j}^{4} \underline{r}_{q+1, j}
$$


into $\underline{M}_{(2 i)}^{(9+1)}$ and re-name the dummy variables of integration from $\left(\underline{\underline{r}}_{n+1 ; j}\right)$ to $\left(\underline{r}_{i j}\right)$, then $\underline{M}_{(2) i}^{(a+1)}$ becomes

$$
\underline{M}_{(2) i}^{(q+1)}=-\frac{1}{2} \int \prod_{k} d \underline{r}_{1 k} n^{(q+1)}\left(\underline{r}_{i}-\frac{q}{q+1} \underline{r}_{1, q+1}+\frac{1}{q+1} \sum_{i} \underline{r}_{1 i}, \underline{r}_{12} \cdots \underline{r}_{1, q+1}\right)
$$

In the same way, we substitute

$$
\underline{x}^{(q+1)}=\underline{r}_{1}+\frac{1}{q+1} \sum_{k} \underline{r}_{1 k}
$$

into ${ }^{M}(1)_{i}^{(1+1)}$ to obtain

$$
\underline{M}_{(1) i}^{(q+1)}=\frac{1}{2} \int \prod_{k} d \underline{r}_{1 k} n^{(q+1)}\left(\underline{r}_{1}+\frac{1}{q+1} \sum_{i} \underline{r}_{1 i}, \underline{r}_{12} \cdots \underline{r}_{1, q+1}\right) \quad 2.39 .
$$

The last step of the proof consists in expanding the integrands of $\underline{M}_{(1) i}^{(9+1)}$ and $\underline{M}_{(2) i}^{(9+1)}$ about $\underline{\underline{r}}_{1}$, the result is

$$
\int \prod_{x} d \underline{\underline{r}}_{1 K} \underline{M}_{-1}^{(q+1)}=\frac{1}{2} \frac{\partial}{\partial \underline{\underline{r}}_{1}} \int \prod_{\kappa} d \underline{r}_{1 K} \hat{\underline{r}}_{1, q+1} \hat{\underline{r}}_{1, q+1} n^{(q+1)}\left(\underline{r}_{1} \ldots r_{1, q+1}\right) r_{1, q+1} \frac{\partial}{\partial r_{1}} \phi_{1, q+1}
$$

The linearization of Eq. (2.33) is now a straightforward operation. We find that

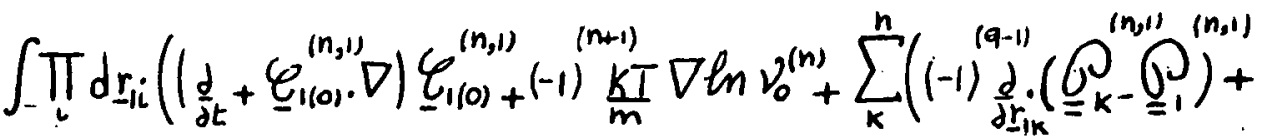

$$
\begin{aligned}
& \left.\left.\sum_{J}^{n}(-1)^{(n-1)} \sum_{P_{0}^{(1, n)}}\left(\underline{Q}_{k}^{(n, j)} Q_{\underline{1}}^{(n, j)}\right) \cdot \frac{\partial}{\partial r_{-1 k}} \ln V_{0}^{(0)}+\underline{M}_{k(0)}^{(q+1)}\right)\right)=0 \\
& \text { where } \underline{Q}_{k}^{(n, m)}=\sum_{c}^{n-m}(-1)^{(1-1)} \sum_{p_{l}^{(m+1, g n)}}\left\langle\underline{V}_{k}^{(m+i)} \underline{V}_{k}^{(m+i)}\right\rangle
\end{aligned}
$$

and $\underline{Q}_{k}^{(n, m)}$ is related to $\underline{Q}_{k}^{(n, 1)}$ in the same way $\underline{\varphi}_{k}^{(n, m)}$ is to $\underline{\mathscr{C}}_{k}^{(n, 1)}$.

Eq. $(2.1,0)$ is the equation that must be satisfied by $\left(\underline{Q}_{k}^{(n, 1)}-\underline{Q}_{1}^{(n, 1)}\right)$

For the case $n=2$, Iiq. (2.40) becomes 
27

$$
\begin{aligned}
& \int_{\underline{\underline{r}}} n_{0}^{(2)}\left\langle\underline{V}_{2}^{(2)} \underline{v}_{2}^{(2)}-\underline{v}_{1}^{(2)} \underline{v}_{1}^{(2)}\right\rangle^{(2)}+\frac{n_{0}^{(2)}}{n} \cdot \underline{\underline{r}} \frac{n_{k}}{m}\left(T_{2}-T_{1}\right) \underline{U}+\frac{n_{0}^{(2)} k T}{m} \nabla \ln V_{0}^{(2)}+ \\
& \frac{2 n}{m}\left(1-\nu_{0}^{(2)}\right) \nabla(p-n k T)+\sum_{k}^{2} \int d \underline{r}_{3}\left(n_{0}^{(3)}-n_{0}^{(2)} n^{(1)}\right) \int_{\underline{r}_{k}} \phi_{k, 3}=0 \quad 2.41
\end{aligned}
$$

With the help of the identity

$$
\partial_{\underline{r}} n_{0}^{(2)}\left\langle\underline{C}_{2} \underline{C}_{-2} \underline{C}-\underline{C}_{1}\right\rangle^{(2)}=\frac{k}{m} r \cdot \nabla T \frac{\partial}{\partial \underline{r}} n_{0}^{(2)}+\frac{k n}{m}{ }_{0}^{(2)} \nabla T
$$

where $\underline{C}=\underline{-}-\underline{u}$, we transform Eq. (2.41) into the equation that in section 2.4, will be used to find the expression for the correlated temperatures.

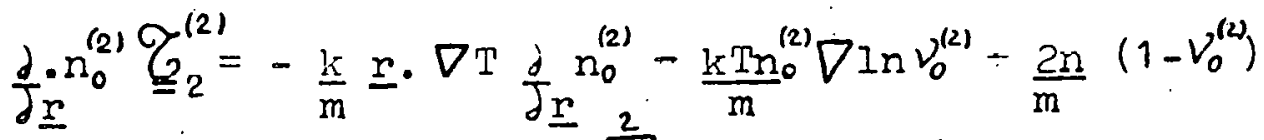

$$
\begin{aligned}
& \nabla(p-n k T)-\sum_{k}^{2} \int \underline{r}_{3}\left(n_{0}^{(3)}-n_{0}^{(2)} n_{1}\right) \frac{\partial}{\partial \underline{r}_{k}} \varphi_{3, k} \quad 2.42 \\
& \underline{\mathcal{G}}_{k}^{(n)}=\left\langle\left(\underline{V}_{k}^{(n)} \underline{V}_{k}^{(n)}-\underline{V}_{1}^{(m)} \underline{\underline{V}}_{1}^{(n)}\right)-\left(\underline{C}_{k} \underline{C}_{k}-\underline{C}_{1} \underline{C}_{1}\right)\right\rangle^{(n)}
\end{aligned}
$$

Note that IG. (2.42) agrees with Eq. (2.17) to terms linear in $\nabla$.

Finally we consider the generalized energy equation. If we define the correlated temperatures by

$$
\frac{3}{2} n^{(9)} k T_{k}^{(a)}=\int_{n}\left(\prod_{i}^{q} d_{i}\right) r_{2}^{2} m\left(\underline{v}_{k}-\underline{u}_{k}^{(9)}\right)^{2}
$$

2.43

then

$$
\tau_{k}^{(n, 1)}=\sum_{q}^{n}(-1)^{(q-1)} \sum_{P_{q}^{(1, n)}} T_{k}^{(q)}=\frac{m}{k} \underline{\underline{U}}: \underline{O}^{(n, 1)}
$$

The starting point in the derivation of an expression to be substituted into the r.h.s. of

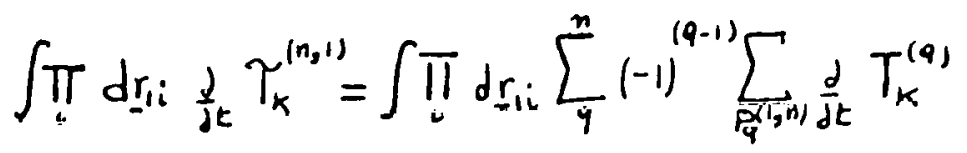


is the equation produced by subtracting Eq. (2.14) from Eq. (2.1.6): Namely,

$$
\begin{aligned}
& \sum_{k}^{q}\left(\left(\frac{\partial}{\partial t}+\sum_{J}^{q} \underline{u}_{j}^{(9)} \cdot \frac{\partial}{\partial r}\right)\left(\frac{3 k}{2} J_{k}^{(9)}+\frac{m}{2} \cdot u_{k}^{(9)}\right)^{2}\right)+\frac{\partial}{\partial r_{k}} \cdot n^{(9)} \dot{T}_{k}^{(9)} \underline{u}_{k}^{(9)}+w_{k}^{(9)}+w_{k}^{(9+1)}=02.45 \\
& W_{k}^{(9)}=n^{(9)} \underline{u}_{k}^{(9)} \cdot \sum_{\underline{\underline{x}}_{k}} \Phi^{(9)}, \quad \Phi^{(9)}=\sum_{L} \sum_{J} \Phi_{i j} \\
& W_{k}^{(q+1)}=\int d \underline{\underline{r}}_{q+1} n^{(q+1)} \underline{\underline{u}}_{k}^{(q+1)} \cdot \frac{\partial}{\partial \underline{\underline{r}}_{k}} \Phi_{k, q+1}
\end{aligned}
$$

The main problem in the linearization of Eq. (2.45) is to show that to linear terms in $\nabla, W_{k}^{(9)}\left(n^{(9)}\right)$ and $W_{k}^{(9+1)}\left(n^{(9+1)}\right)$ ean be replaced by $W_{k(0)}^{(\theta)}\left(n_{0}^{(1)}\right)$ and $W_{k(0)}^{(p+1)}\left(n_{0}^{(\theta+1)}\right)$, their equilibrium forms. This can be easily done with the help of Eq. (2.14). In fact

$$
\begin{aligned}
& \sum_{k} \int \prod_{i} d \underline{r}_{i} W_{k}^{(9)}=\int \prod_{i}^{q} d \underline{r}_{1 i}\left(\Phi^{(9)} \frac{\partial}{\partial t} n^{(9)}+\sum_{k} \frac{\partial}{\partial r_{k}} \cdot n^{(9)} \underline{u}_{k} \Phi^{(9)}\right)
\end{aligned}
$$

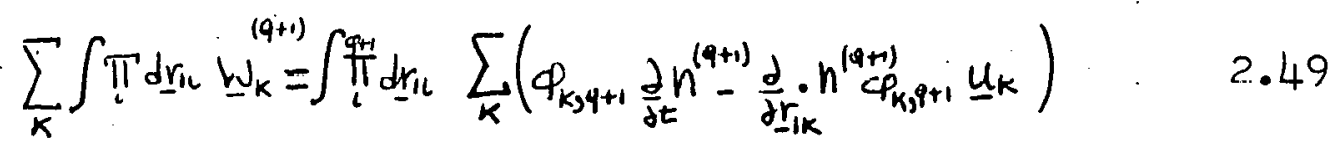

The Iinearization of Eqs. $(2.48,2.49)$ and of the other terms in Eq. (2.44) is staightforward After substitution

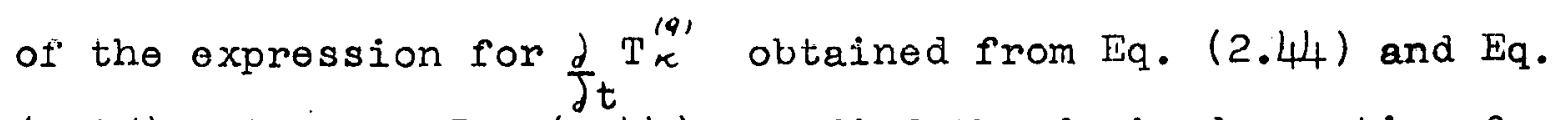
$(2.15) \mathrm{n}=1$, into $\mathrm{Eq}$. $(2.44)$, we find the desired equation for $\tau_{k}^{\left(n^{\prime \prime}\right)}$

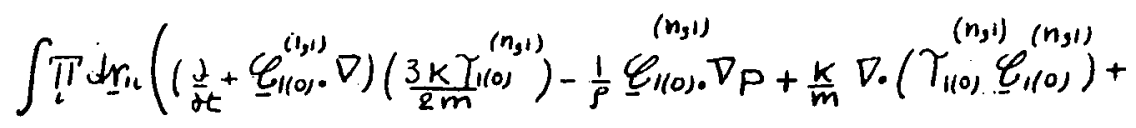

$$
\begin{aligned}
& \left.(-1) \frac{k}{m} \tau_{1(0)}^{(n+1)} \mathscr{\mathscr { E }}_{1(10)}^{(n, n)} \cdot \nabla \ln V_{0}^{(n)}+\tilde{W}_{k(0)}^{(n)}+\tilde{W}_{k(0)}^{(n+1)}+\mathcal{T}_{(0)}^{(n)}\right)=0
\end{aligned}
$$


where

$$
\begin{aligned}
& \tilde{W}_{K(0)}^{(n)}=\sum_{9}^{n}(-1) \sum_{P_{9}^{(1, n)}}^{(a-1)}\left(W_{k(0)}^{(n)}\right) \\
& \tilde{W}_{k(0)}^{(n+1)}=\sum_{q}(-1) \sum_{P_{Q}(1, n)}^{(9-1)}\left(W_{K(0)}^{(n+1)}\right) \quad 2.52
\end{aligned}
$$

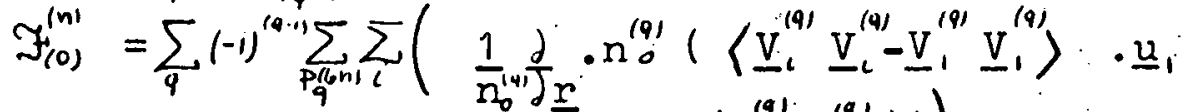

$$
\begin{aligned}
& \left.\left.+T_{1}\left(\underline{u}_{L}^{(q)}-\underline{u}_{1}^{(q)}\right)\right)\right)
\end{aligned}
$$

For $\mathrm{n}=1$, Eq. (2.50) yields the equation that in section 2.3 we will use to derive Euler's energy equation,

$$
\left(\frac{\partial}{\partial t}+\underline{u}_{i} \nabla\right)\left(\frac{3 k T)}{2 m}+\frac{k T}{m} \nabla \cdot \underline{u}_{1}-\frac{1}{\rho} \cdot \underline{u}_{i} \nabla(p-n k T)+\tilde{w}_{(10)}^{(2)}=02.54\right.
$$

Eq. (2.54) agrees with Eq. (2.17), $k=3$. In Eq. (2.50) the $\frac{\partial}{\partial t} n^{(a)}$ terms are clearly proportional to the gradients since the time (and position) dependence of $n^{(\theta)}$ is through the macroscopic parameters $n$ and $T$. Since we know $\mathcal{F}^{(n)}$ from Eqs. $(2.30,2.40)$, we have proved that the consistency of the generalized continuity and momentum equation, guarantee the consistency of the generalized energy equation.

Having established the existence of a set of differential equations that the first three moments of $f_{0}^{(n)}: \underline{\mathscr{C}}^{(n, 1)} \rho^{\left(n_{1}\right)}$. and $\tau^{(n, y)}$ must satisfy, we consider the problem of constructing an expression for $f_{0}^{(n)}$ consistent with all the velocity moments in Eqs. $(2.30,2.40,2.50)$ and with conditions $(2.14$, 2.20). From the definition of $n_{0}^{(n)}$ and the identity in E'q. $(2.21)$, we let

$$
f_{0}^{(n)}=\frac{1}{n} \prod_{k}^{n} f_{\left.O_{k}\right)}^{(1, n)} \prod_{j} \prod_{\rho_{j}(1, n)} \nu_{0}^{(j)}
$$


where $\prod_{P(k, n)}$ indicates the products of all the possible ways a cluster of $j$ molecules can be chosen from $k+1, k+2 \ldots n$ molecules. The first few members of the set $\left(f_{0}^{(n)}\right)$ are

$$
\begin{aligned}
& f_{0}^{(1)}=f_{0}^{(1)} \\
& f_{0}^{(2)}=f_{01}^{(1,2)} f_{02}^{(1,2)} \gamma_{0}^{(2)} \\
& f_{0}^{(3)}=f_{01}^{(1,3)} f_{02}^{(1,3)} f_{03}^{(1,3)} \nu_{012}^{(2)} \gamma_{0,3}^{(2)} \nu_{023}^{(2)} \nu_{0123}^{(x)} \\
& f_{0}^{(4)}=f_{01}^{(1,4)} f_{02}^{(1,4)} f_{03}^{(1,4)} f_{04}^{(1,4)} V_{012}^{(2)} \nu_{0,3}^{(2)} \nu_{014}^{(1)} V_{023}^{(21} V_{024}^{(2)} \\
& \text { - } \quad \nu_{034}^{(2)} \nu_{0123}^{(3)} V_{0124}^{(3)} \nu_{0134}^{(3)} \nu_{0234}^{(3)} V_{01234}^{(4)}: 2.56
\end{aligned}
$$

and by definition

$$
\begin{aligned}
& n_{0}^{(1)}=n_{1}^{(1)} \\
& n_{0}^{(2)}=n_{1}^{(1)} n_{2}^{(1)} \nu_{0}^{(2)} \\
& \vdots
\end{aligned}
$$

Then we choose a form for the correlated singlet velocity distribution functions $\left(f_{a n}^{(1, n)}\right)$ that will make $f_{0}^{(n)}$ as defined in Eq. (2.56) consistent with the moments $n_{0}^{(n)}, n^{(n)} \underline{u}_{o l}^{(n)}, T_{0 L}^{(n)}$ and $\mathscr{q}_{l}^{(n)}$ appearing in Eqs. $(2.30,2.40,2.50)$.

A pair diatribution function with the properties mentioned above can bc obtained by expanding $f_{a c}^{(1, n)}$ in terms of an orthogonal set of irreducible tensors as

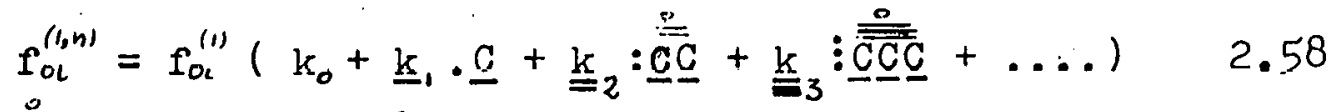

$$
\begin{aligned}
& \underline{\underline{\underline{C}}}=\underline{C} \underline{C}-1 / 3 C^{2} \underline{U} \\
& 2.59 \\
& \underline{\underline{C} \underline{\bar{C}} \underline{C}}=\underline{C} \underline{C} \underline{C}-1 / 5(\underline{C}+\underline{\underline{U}} \underline{\underline{C}}+(\underline{C})) \\
& 2.60
\end{aligned}
$$


and then by choosing the expansion coefficients $\left(k_{i}\right)$ so that $f_{0}^{(n)}$ satisfies all the velocity moments in Eqs. $(2.30,2.40$, 2.50). If we expand the scalar function $k_{0}$ in Sonine polynomials (27) as

$$
\begin{aligned}
k_{0} & =\sum_{i=0}^{\infty} c_{1} s_{1 / 2}^{(1)}\left(w^{2}\right) \\
& =c_{0}+c_{1}\left(3 / 2-w^{2}\right)+c_{2}\left(1 / 2 w^{4}-5 / 2 w^{2}-15 / 8\right)+\ldots 2.61
\end{aligned}
$$

where

$$
S_{n}^{(m)}(y)=\sum_{p=0}^{m}(-y)^{p}(m+n) ! /((n+p) !(m-p) ! p !)
$$

then the conditions on $f_{o c}^{(1, n)}$ in Eqs. $(2.43,2.57)$ require that

$$
\begin{aligned}
& k_{0}=1+\left(\mathrm{m} / 3 k \mathrm{KT}_{01}^{(n)}\right)\left(\mathrm{w}^{2}-3 / 2\right)\left[\mathrm{c}^{2}\right]^{(n)} \\
& {[()]^{(n)}=\langle())^{(n)}-\int \prod_{c}^{n} d \underline{v}_{c} \prod_{k}^{n} f_{0 k}^{(1)}()}
\end{aligned}
$$

To find $\underline{k}_{1}$, we operate on Eq. $(2.58)$ with $\langle\underline{C}()\rangle^{(n)}$

$$
\left\langle\underline{C}_{1}\right\rangle^{(n)}=1 / 3 \underline{k}_{1}\left\langle C^{2}\right\rangle^{(n)}
$$

From the condition in Eq. (2.25) we obtain

$$
\underline{k}_{1}-\left(m / k T_{l}^{(n)}\right)\langle\underline{c}\rangle^{(n)} \quad 2.64
$$

In a similar way we find $\underline{\mathrm{k}}_{2}$

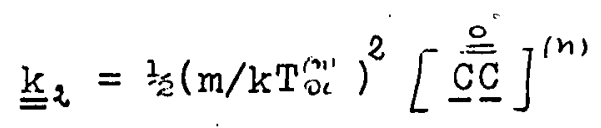

All the ullier coefflcients $\left(k_{1}\right), i>2$ need not be considered since the velocity moments which they are contracted with do not appear in the integrodifferential equations we are 
solving. Furthermore, since higher moments in the expansion of $f_{0}^{(2)}$ do not contribute to the fluxes, only the first thirteen moments will be retained. Truncated in this way, $f_{0}^{(2)}$ can be written in a Maxwellian form as

$$
\begin{aligned}
& f_{a l}^{(1,2)}=n_{c}\left(m / 2 \pi k \mathrm{~T}_{c}^{(2)}\right) e^{-\left(\underline{\beta}: \underline{V}_{c}^{(2)} \underline{V}_{c}^{(2)}\right)} \\
& \underline{\underline{\beta}}=\left(\mathrm{m} / 2 \mathrm{kT}_{\mathrm{c}}^{(2)}\right)\left(\underline{\underline{U}}+\frac{\mathrm{m}}{k \mathrm{~T}_{c}^{(2)}}\left[\underline{\underline{\underline{\mathrm{C}}}} \underline{\underline{C}}_{i}\right]\right)
\end{aligned}
$$

If we expand Eq. (2.66) about $f_{o c}^{\prime \prime \prime}$ and neglect terms not linear in $\nabla$, we obtain E'q. (2.58).

Next we want to verify that Eq. (2.58) is consistent with $\underline{\tau}_{c}^{(n)}$. To evaluate this moment we need to solve integrals whose integrands involve the components of $4^{\text {th }}$ rank isotropic tensors. Since there are only three $4^{\text {th }}$ rank isotropic tensors, we let

$$
\begin{aligned}
& \int d \underline{\hat{C}} \underline{\hat{C}} \hat{\underline{C}} \hat{C} \hat{C}=a_{1} \underline{\underline{U}}+a_{2} \underline{\underline{\underline{E}}}+a_{3} \underline{\underline{\underline{E}}} \quad, \hat{C}=\underline{\underline{C}} / \mathrm{C} \quad 2.67 \\
& (U U)_{1 j k e}=\delta_{1 j} \delta_{k e},(W)_{1 j k j}=\delta_{1 x} \delta_{j e},(U)_{1 j k e}=\delta_{1 k} \delta_{J K}
\end{aligned}
$$

The coefficients $a_{1}, a_{1}, a_{3}$ are found by alternatively double dotting UU, $W$ and $\Psi$ into Eq. (2.67). From the solution of the resulting set of three equations in three unknowns, we find that $a_{1}=a_{2}=a_{3}=4 \sqrt{\pi} / 15$. With the result in Jiq. (2.67) to verify that $f_{0}^{(2)}$ is consistent with the full second velocity moment $\underline{\tau}^{\prime \nu}$ is lengthy but simple. We have left to verify that $f_{0}^{(n)}$, as defined in Eq. (2.58), is consistent also with condition (2.20), this will be done in section 3.3 . 
We discus's next the conditions that must be obeyed by $f_{c}^{(n)}$. With the condition in Eq. (2.17), Eq. (2.13) yields

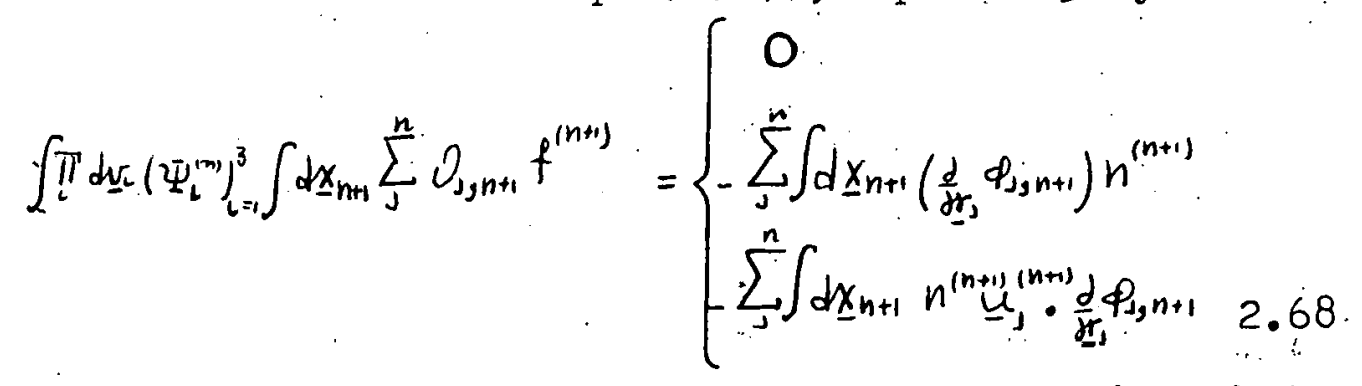

The probability of finding a molecule in $\underline{\underline{r}}_{n+1}$ knowing that there is a molecule in $d \underline{r}_{1}$, a molecule in $d \underline{r}_{2} \ldots$ a molecule in $d \underline{\underline{r}}_{n}$, is $\left(\mathrm{m}^{(n+1)} / \mathrm{n}^{(n)}\right) d \underline{\underline{r}}_{n+1}$. If $\phi_{j, n+1}$ is the interaction potential between molecule $j$ and molecule $n+1$, then

$$
\begin{aligned}
& \underline{F}_{j}^{(n+1)}=\int d \underline{\underline{r}}_{n+1}\left(n^{(n+1)} / n^{(n)}\right)\left(-\sum_{\underline{r}_{j}} \phi_{j, n+1}\right) \\
& W_{j}^{(n+1)}=\int d \underline{r}_{n+1}\left(n^{(n+1)} / n^{(n)}\right) \underline{u}_{j}^{(n)} \cdot\left(-\frac{\partial}{\partial \underline{r}_{j}} \varphi_{j, n+1}\right) \quad 2 \cdot 70
\end{aligned}
$$

are the average force that the molecules which are not in the $n$-particle cluster exert on the molecule $j$ and the average work done by the molecules on the molecule $j$. Then

$$
\begin{array}{ll}
\underline{I}^{(n+1)}=\sum_{1} \underline{F}_{j}^{(n+1)} & 2.71 \\
W^{(n+1)}=\sum_{J} W_{J}^{(n+1)} & 2.72
\end{array}
$$

are the average force and work done by $N-n$ molecules on a cluster of n-molecules. In term of Eqs. $(2.71,2.72)$ the r.h.s. of Eq. (2.68) becomes

$$
\text { r.h.s. }\left\{\begin{array}{lll}
0 & i=1, & n>1 \\
n^{(n)} \underline{F}^{(n+1)} & i=?, & n>1 \\
n^{(n)} W^{(n+1)} & i=3, & n>1
\end{array}\right.
$$


The decomposition of $f^{(n)}=f_{0}^{(m)}+f_{c}^{(n)}$, and the Eq. in (2.17) impose on $f_{c}^{(n)}$ the condition

$$
\int \Pi d \underline{v}_{L}\left(\Psi_{k}^{(n)}\right)_{k=1}^{3}\left(\sum_{1}^{n}\left(\underline{v}_{1}, \cdot \frac{\partial}{\partial r_{1}} f_{c}^{(n)}-\sum_{i} \partial_{i_{j}} f_{c}^{(n)} \int d \underline{r}_{n+1} O_{\perp g} n+1 f_{c}^{(n+1)}\right)\right)=0 \quad 2.74
$$

When $n=1, E q .(2.74)$ implies that

$$
n_{c}^{(2)} W_{c}^{(2)}=0=n_{c}^{(2)} \underline{F}_{c}^{(2)}
$$

In general, however, Eq. (2.75) does not hold and Eqs. (2.19, 2.74) are the conditions that $f_{c}^{(n)}$ must satisfy. In section 3.1 we will find an expression for $f_{c}^{(2)}$ which will satisfy Eq. $(2.74)$. In sections $3.3,3.4$ we will show that the expression for $f_{c}^{(2)}$ derived from Eq. (2.74) is consistent with condition (2.20).

From the definition of $f_{0}^{(2)}$, we note that in this theory, the effect of position correlations between molecules is introduced by $\nu_{0}^{(2)}$. , the equilibrium radial distribution function and that the effect of velocity correlations is introduced by defining the singlet velocity distribution functions in terms of correlated parameters, as in Eq. (2.58). Since we want to calculate first density corrections to transport coefficients due only to spacial inhomogeneity and velocity correlations, all $\mathrm{n}^{3}$ terms in $\mathrm{f}_{0}^{(2)}$ will be disregarded by making $\nu_{0}^{(2)}$ density independent. The truncation of $\nu_{0}^{(i)}$ will cause the coefficient of bulk viscosity to disappear. 


\subsection{Euler's Equations for an Imperfect Gas}

If we let $n=1$ in E'qs. $(2.30,2.40,2.54)$, the generalized conservation equations reduce to Euler's hydrodynamic equations for an imperfect gas. These are

$$
\begin{aligned}
& \frac{\partial}{\partial t} n+\nabla \cdot n \underline{u}=0 \\
& \left(\frac{\partial}{\partial t}+\underline{u} \cdot \nabla\right) \underline{u}+\frac{1}{\rho} \cdot p \underline{\underline{U}}=0 \\
& \left(\frac{\partial}{\partial t}+\underline{u} \cdot \nabla\right)\left(\frac{1}{2} u^{2}+\tilde{E} \cdot\right)+\frac{1}{\rho} \nabla \cdot \underline{u} p=0
\end{aligned}
$$

Here the hydrostatic pressure $\mathrm{p}$ and the internal energy per unit mass $\tilde{E}$, corrected to include the contributions of the interactions to the thermodynamic functions of the gas, are

$$
\begin{aligned}
& p=n k T-\frac{1}{6} \int d \underline{r} n_{0}^{(2)} \frac{\partial}{\partial r} \varphi(r) \\
& \bar{E}=\frac{3 n k T}{2}+\frac{1}{2} \int d \underline{r} n_{0}^{(z)} \varphi(r)
\end{aligned}
$$

Eq. (2.76) could have been obtained directly from the Boltzmann equation since interactions, characteristic of a real dense gas, do not have any consequence on the conservation of mass. In a dense gas, interactions do affect the stress tensor. Furthermore, it is the sum of the kinetic and potential energy and not $\frac{1}{2} \mathrm{mv}^{2}$ which is a collision invariant. Therefore, it is no surprise that the Euler momentum and energy equations cannot be obtained directly from the first and trace of the second velocity moments of the first 
BBGKY equation without assuming the form of $f_{0}^{(2)}$ given in $\mathrm{Eq}$. (2.58). We have thus shown that the nondissipative hydrodynamic equations (which are Hilbert's integrability conditions) are satisfied if $f_{0}^{(2)}$ is of the form given in Eq. (2.58). The advantages and the motivation behind the decomposition of $f^{(2)}=f_{0}^{(2)}+f_{c}^{(2)}$ become now apparent. For we now notice that the expression for the correlated parameters are specified in terms of certain well known results of the statistical mechanics of equilibrium in which the parameters of the equilibrium state are replaced by parameters of the local equilibrium state.

\subsection{The Correlated Velocities and the Temperature Tensors}

The equation to be used to find $\underline{u}^{(2)}=\nu_{0}^{(2)}\left(\left(\underline{u}_{2}^{(2)}-\underline{u}_{1}^{(2)}\right)-\left(\underline{u}_{1}-\underline{u}_{2}\right)\right)$ was derived in section 2.2. In Eq. (2.32) we make use of the thermal energy equation (28) to replace the streaming (or convective) part with a term proportional to $\nabla$.

$$
\frac{\partial}{\partial \underline{\underline{r}}} \cdot \underline{u l}^{(2)}=-\bar{g}(r) \nabla \cdot \underline{u}-h(r) \underline{\underline{\underline{r}}} \underline{\underline{r}}: \underline{S}
$$

where

$$
\begin{aligned}
& g(r)=\frac{1}{3} r \frac{\partial}{\partial r} \nu_{0}^{(2)}+\frac{\nu_{0}^{(1)} I n \nu_{0}^{(1)}}{m n C_{v}}\left(\frac{\partial P}{\partial T}\right)_{v} \\
& h(r)=r \frac{\partial}{\partial r} \nu_{0}^{(2)} \\
& S_{i j}=\underline{\underline{r}}\left(\nabla_{i} \underline{u}+\nabla_{j} \underline{u}_{v}\right)-\frac{1}{3} \nabla \cdot \underline{u} \delta_{i j}
\end{aligned}
$$

$C_{v}$ is the specific heat per unit mass. The mathematical problem represented by the solution of $\mathrm{Eq} .(2.81)$ can be 
formulated as follows. Given the first order partial differential equation

$$
\frac{\partial}{\partial \underline{\underline{r}}} \cdot \underline{\mathcal{U}}^{(z)}=G(\underline{\underline{r}})
$$

where $G(\underline{r})$ is the r.h.s. of Eq. (2.81) we wish to find the vector. $\underline{u}^{(2)}$ subject to the conditions

$$
\begin{array}{lll}
\operatorname{Lim}_{x \rightarrow 0} \underline{u}^{(2)}=0 & 2.85 \\
\lim _{r \rightarrow \infty} \underline{u}^{(2)}=0 & 2.86
\end{array}
$$

Condition (2.85) is imposed by the spacial correlation function $V_{0}^{(2)}$, while the boundary condition $(2.86)$ is due to the disappearance of velocity correlations contained in $\underline{u}^{(2)}$ at large separation.

Provided that the integral

$$
\underline{g}(\underline{r})=\int d \underline{x} \frac{\underline{u}(2)}{\mid \underline{x})}
$$

exist, then $\underline{u}^{(2)}$ can be uniquely divided into a solenoldal and irrotational part as follows: (29)

$$
\begin{aligned}
& \underline{u}^{(2)}=\frac{\partial}{\partial \underline{r}} C(\underline{r})+\frac{\partial}{\partial \underline{r}} \times \underline{D}(r)
\end{aligned}
$$

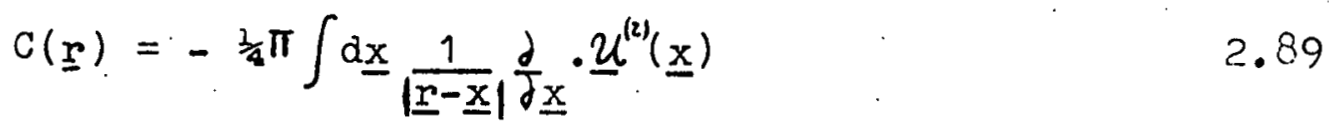

$$
\begin{aligned}
& \underline{D}(\underline{\underline{r}})=+\frac{1}{4} \pi \int \partial \underline{\underline{x}} \frac{1}{|\underline{r}-\underline{\underline{r}}|} \frac{\partial}{\partial \underline{x}} \times \underline{u}^{(\xi)}(\underline{x}) \quad 2.90
\end{aligned}
$$

We now derive sufficient conditions for the existence of $\underline{g}(\underline{r})$ as defined by Eq. (2.87). After changing the integration 
variables to $\underline{z}=\underline{x}-\underline{r}$ and using polar coordinates we obtain

$$
\underline{q}(\underline{\underline{r}})=\int_{0}^{2 \pi} \mathrm{d} \Phi \int_{0}^{\pi} \mathrm{d} \theta \sin \theta \int_{0}^{\infty} \mathrm{dz} \underline{u}^{(2)}(\underline{z}+\underline{\underline{r}})
$$

If the limit

$$
\operatorname{Lim}_{z \rightarrow \infty} z^{3} \underline{u}^{(z)}
$$

exists and $\underline{\mathcal{U}}^{(2)}$ is bounded then $\underline{G}$ is a convergent integral. From the properties of $\underline{U}^{(2)}$ described in Eqs. $(2.85,2.86)$ we conclude that Eq. (2.88) is the general solution of Eq. (2.84). With $\theta$ and $\varphi$ representing the polar angles of $\underline{x}$ with respect to $\underline{\underline{r}}$, the expression for $C(\underline{\underline{r}})$ can be simplified to a one dimensional integral. In Eq. (2.89) we substitute the r.h.s. of Eq. (2.84) for $\nabla . \mathcal{U}^{(2)}$. Since $0<\varphi<2 \pi$ and $0<\theta<\pi$, all terms odd in $\cos \theta$, sine are neglected; the integration over $\varphi$ is easily done, we find

$$
C(\underline{r})=\frac{1}{4} \int_{-1}^{t} d(\cos \theta) \int_{0}^{\infty} d x x^{2} \frac{h(x)\left(3 \cos ^{2} \theta-1\right) \hat{r} \hat{r}: S+2 g(x)}{|\underline{x}-\underline{\underline{r}}|} \nabla \cdot \underline{u}
$$

The form of the integrand of $C(\underline{r})$ suggests to expand $|\underline{x}-\underline{r}|^{-1}$ in terms of Legendre polynomials. For two vectors $\underline{m}$ and $\underline{n}$ where $|\underline{m}|>|\underline{n}|$

$$
|\underline{m}-\underline{n}|^{-1}=\frac{1}{m}\left(1+\left(\frac{n}{m}\right) \cos \theta+\frac{1}{2}\left(\frac{n}{m}\right)^{2}\left(300 \sin ^{2} \theta-1\right)+\ldots \ldots\right)
$$

After decomposing the integration over $x$ into the intervals $0<x<r$ and $r<x<\infty$, with Eq. (2.91) C( $\underline{r})$ becomes

$$
\left.C(\underline{r})=\frac{1}{5} \underline{\underline{r}} \underline{\underline{r}}: \underline{S}\left(\frac{h_{1}(r)}{r^{5}}-h_{2}(r)\right)+\frac{\left(g_{1}(r)\right.}{r}-g_{2}(r)\right) \nabla \cdot \underline{u}
$$


where: $h_{1}(r)=\int_{0}^{r} d x x^{4} h(x) ; h_{2}(r)=-\int_{r}^{\infty} d x h(x) / x$ $g_{1}(r)=\int_{0}^{r} d x \cdot x^{2} g(x) ; g_{2}(r)=-\int_{r}^{\infty} d x x g(x)$

from which we obtain the irrotational part of $\underline{u}^{(2)}$

$$
\left.\left.\int_{\underline{\underline{r}}} C(\underline{\underline{r}})=\frac{h_{1}(r)}{r^{4}}\right) \underline{\hat{r}} \underline{\underline{r}} \underline{\underline{r}}: \underline{S}-\frac{2}{5} \underline{\underline{r}} \cdot \underline{=} \frac{\left(h_{1}(r)\right.}{r^{5}}-h_{2}(r)\right)-\frac{\hat{r} g_{1}}{r^{2}}(r) \nabla \cdot \underline{u} \quad 2.91 \%
$$

The solenoldal part of $\underline{U}^{(2)}$ cannot be determined from Eq. (2.90). We defer discussion of this point to section 2.6 .

To find the correlated temperatures we must solve Eq. (2.41). The comments made in regard to the equation involving the divergence of the correlated velocities, apply also in this case. Now the mathematical problem consists in finding nine unknowns (the components of $\underline{\tau}^{(2)}$ ) from three equations (the components of the vector on the r.h.s. of Eq. (2.42)). The same arguments used in the derivation of a general solution of Eq. (2.84) can be used to solve Eq. (2.42) for $\underline{\underline{Z}}^{(2)}$. In terms of their components Eq. (2.42) decomposes as

$$
\begin{aligned}
& \underline{\partial}_{\underline{r}} \cdot \underline{t}_{k}=F_{k} \\
& \underline{t}_{k}-\sum_{J} \tau_{k k} \underline{\hat{\theta}}_{J}
\end{aligned}
$$

$F_{k}$ is the kth component of the vector in the r.h.s. of Eq. $(2.84), \tau_{j k}$ is a component of the tensor $\underline{\tau}^{(2)} \underline{\hat{\theta}}_{j}$ is a unit vector. Then the similarity of Eq. (2.92) to Eq. (2.84) is evident; from liq. (2.92) it follows that

$$
\underline{\tau} \stackrel{(2)}{=} \frac{\partial}{\partial \underline{\underline{C}}}(r)+\frac{\partial}{\partial \underline{\underline{x}}} \mathrm{I} \stackrel{\tilde{D}}{=}(r)
$$




$$
\text { where } \begin{array}{rlr}
\tilde{\underline{C}}(\underline{\underline{r}}) & =\sum_{k} \tilde{C}_{k} \underline{\hat{e}}_{k} & 2.95 \\
\underline{\underline{D}}(\underline{\underline{r}}) & =\sum_{k} \underline{D}_{k} \hat{\hat{e}}_{k} & 2.96
\end{array}
$$

and $\widetilde{C}_{k}$ and $\tilde{D}_{k}$ are defined as in Eqs. $(2.89,2.90)$ with $\underline{t}_{k}$ replacing $\underline{u}^{(2)}$

2.5 A General Outline of a Method of Procedure to Evaluate Transport Coefficients for a Dense Gas

As mentioned in chapter 1, the main problem in the statistical mechanical approach to the kinetic theory of gases, is the derivation of closed kinetic equations from the Liouville's equation. The BBGKY equations are customarily taken as a starting point for the solution of this problem. A formally exact generalization of Boltzmann's equation can be derived for purely repulsive potentials if it is assumed that at time $t=-\infty$ no correlations existed(11). Kinetic ëquations obtained from this procedure do not contain effects due to molecular bound states and lead to transport coefficients which are not in satisfactory agreement with experiment for real gases at low temperatures where presumably bound states play an important role.

The BBGKY equations are all intercorrelated. In fact, to solve the nth equation of the hierarchy we must know $f^{(n+1)}$ which is the function which satisfies the nth+1 equation. One way to proceed in determining the set of functions $\left(f^{(k)}\right)_{k=1}^{n}$ is to truncate the hierarchy at a certain level, say the nth+1 
level, by approximating $f^{(n+1)}$ in terms of lower order distribution functions and then solve the coupled but closed set of hierarchy equations for the functions $\left(f^{(k)}\right)_{k=1}^{n}$.

In this dissertation we have presented an alternative scheme for obtaining the set of functions $\left(f^{(k)}\right)_{k=1}^{n}$. In chapter 2, we have shown that one can define a set of functions $\left(f_{0}^{(k)}\right)_{k=1}^{n}$ of equilibrium form which contain certain exact velocity moments of $\left(f^{(k)}\right)_{k=1}^{n}$. These moments satisfy the many-body conservation equations for particle density, momentum and energy and, in principle. can be obtained from the solution of these equations. Ilaking the substitution $f^{(n)}=f_{0}^{(n)}+f_{c}^{(n)}$ and $I$ inearizing the equations in the macroscopic gradients, the BBGKY hierarchy then becomes a set of equations governing the unknown function $\left(f_{c}^{(k)}\right)_{k=1}^{m}$. This hierarchy is of course also coupled, but we assume that by some suitable approximation it can be un-coupled so that the unknown functions $\left(f_{c}^{(1)}, f_{c}^{(2)} \ldots f_{c}^{(n)}\right)$ obey a closed set of equations from which they can be determined. This procedure is presumably preferable to other possible truncation schomo in that it exarti.y includes those correlations necossary to derlve the n-particle conservation equations.

By definition the moments of $f^{(1)}$ are related to $n$, $\underline{u}$ and I, hence we required that the velocity moments of the kinetic equation (the 1st BBGKY equation) involving macroscopic parameters, must give the equations of change of the gas. 
Hydrodynamic equations from the BBGKY hierarchy differ from the ones derived from the low density Boltzmann's equation because all thermodynamic functions like the hydrostatic pressure and internal energy have a contribution due to the existence of molecular interactions.

We have shown that these equations contain functions of the velocity moments of $f_{0}^{(2)},\left(n_{0}^{(2)}, \underline{u}_{o l}^{(2)}, \underline{\tau}^{(2)}\right)$ and that $f_{0}^{(2)}$ is capable of satisfying the conservation equations of the 2nd BBGKY equation to terms linear in the macroscopic gradients, (from this point, all equations will be understood to be valid to linear terms only, unless otherwise specified). The tensors $\underline{u}^{(2)}$ $q^{(2)}$, obey differential equations derived from the velocity moments of the 2nd BBGKY equation. From $\underline{u}^{(2)}$ and $\underline{\tau}^{(2)}$ we could uniquely define $f_{0}^{(2)}$ (and consequently $f_{c}^{(2)}$ ) which can then be used to find $f^{(1)}$, the function that satisfies the 1st BBGKY equation. In this way we would have found $f^{(1)}$ and $f^{(2)}$ the functions needed to correctly calculate transport coefficients. For the hard sphere molecular model, a form of $f_{0}^{(2)}$ can be assumed from the theory of Enskog. Then the tensors $\underline{u}^{(i)}$ and $\underline{\underline{\tau}}^{(2)}$ can be directly ovaluated and their irrotational parts calculated with the help of the differential equations obeyed by $\underline{u}^{(\nu)}$ and $\underline{\tau}^{(\nu}$. By subtracting the irrotational parts from the expressions for $\underline{u}^{(v)}$ and $\underline{\tau}^{(u}$, the solenoidal parts of these tensors can be determined.

In general, however, $\mathrm{f}_{0}^{(2)}$ is an unknown function. We have shown that $f_{0}^{(2)}$ can be expanded in terms of its velocity 
moments and that it satisfies linear conservation equations. Only when its velocity moments are known $f_{0}^{(2)}$ can be said to be uniquely defined. Unfortunately $\underline{u}^{(2)}$ satisfies a scalar equation and $\underline{\tau}^{(v)}$ a vector equation; for this reason the general expression of these tensors is not available.

On the basis of the results obtained for hard spheres, the solenoidal parts of $\underline{u}^{(2)}$ and $\underline{\tau}^{(2)}$ are chosen so that the new expressions for $\underline{\mathcal{u}}^{(2)}$ and $\underline{\tau}^{(2)}$ are particular solutions of the differential equations obeyed by these tensors. In the next chapters we will show that the particular choice of $\underline{u}^{(2)}$ and $\underline{\tau}^{(2)}$ lead to a kinetic equation and transport coefficients in agreement with the theory of Enskog. It will also be possible to verify that the irrotational parts of $\underline{u}^{(2)}$ and $\underline{\tau}^{(2)}$ are the major contributor to the transport coefficients.

\subsection{Correlated Parameters for Hard Spheres}

As we have mentioned in sections 2.4 and 2.5, the correlated tensors $\underline{u}^{(2)}$ and $\underline{\tau}^{(2)}$ can each be decomposed into solenoidal and irrotational parts. It has been previously shown that the irrotational terms can be determined by solving generalized conservation equations with appropriate boundary conditions. However, these equations do not govern the solenoidal terms, which apart from boundary conditions may be arbitrarily chosen. Clearly, in the spirit of the last section, we would like to make this choice in such a manner that the 
plausibility of the truncating approximation on $f_{c}^{(2)}$ is maximal. That is, in a sense, we would like to build into $f_{0}^{(2)}$ as much of the real correlation as possible so that a simple truncating approximation on $f_{c}^{(2)}$ is permissible. Specifically, we would like to retain multibody collisional effects in $f_{0}^{(2)}$ through the correlated parameters and to determine $f_{c}^{(2)}$ from binary collision correlations in the manner of the usual low density Boltzmann equation.

Unfortunately, we have not yet found an unambiguous method to carry out this program, and we are thus forced to choose the solenoidal parts of the correlated parameters in a more indirect and less satisfactory way. In this regard, we will choose as a starting point the hard spheres, dense gas theory of Enskog( 30$)$ which has played an important role in the kinetic theory of dense fluids. For the hard sphere potential the collisional term of the 1st BBGKY equation (i.e.), the r.h.s. of Lq. (3.13)) requires a knowledge of $f_{0}^{(2)}$ only on the collision sphere, which is the sphere in the space of $\underline{r}=\underline{r}-\underline{r}$, for which $\underline{r}$ is infinitesimally greater than the hard sphere diameter. Enskog assumed that $f^{(2)}$ on the collision sphere is of the form

$$
f^{(2)}=f_{1}^{(1)^{\prime}} f_{2}^{(1)^{\prime}} \nu_{0}^{(2)}
$$

where the primed functions are functions of pre-collision velocitios. That is, for configurations of ( $\underline{\underline{r}} \underline{\underline{v}}$ ) in the rel-. ative position-velocity space for which the binary collision 
is imminent $\underline{\underline{v}}=\underline{\mathrm{v}}$. On the other hand, for configurations. where the hard sphere collision has just occurred $\underline{y}^{\prime}$ is the velocity immediately prior to collision. The factor $\mathcal{V}_{0}^{(2)}$ takes into account the scroening of two colliding molecules by other molecules in the system and hence accounts, in an approximate manner, for the effects of multibody interactions. This is obviously an approximation in that nonequilibrium distortions of $\nu_{0}^{(1)}$ are ignored.

In the spirit of the Enskog approximation we assume that $f_{0}^{(2)}$ for the hard sphere model should have the form

$$
f_{0}^{(2)}=f_{01}^{(1)^{\prime}} f_{02}^{(1)^{\prime}} \nu_{0}^{(2)}
$$

which is the best approximation to $f_{0}^{(2)}$ involving only liaxwellian velocity distribution functions. Now $f_{02}^{\prime \prime}$ is a function of the macroscopic position $\underline{r}_{2}=\underline{r}_{1}+\underline{\sigma}$ and $\nu_{0}^{(2)}$ is a function of $\underline{X}=\underline{\underline{s}}+\frac{\sigma}{2}$. Expanding this macroscopic space dependence about $\underline{n}_{1}$ yields

$$
\begin{aligned}
& f_{0}^{(2)}=f_{01}^{(1)} f_{02}^{\prime(1)} \bar{\nu}_{0}^{(2)}\left(1+\underline{\sigma} \cdot \nabla_{\ln } \bar{f}_{02}^{(1)}+\frac{1}{2} \underline{\sigma} \cdot \nabla \ln \bar{\nu}_{0}^{(2)}\right) \\
& \underline{\gamma}=1 / \sqrt{2} \quad\left(\underline{W}_{2}-\underline{W}_{1}\right)
\end{aligned}
$$

$\hat{\sigma}$ is a unit vector along $\sigma$; the bar on top of $f_{c 2}^{\prime \prime \prime}$ and $V_{3}^{(1)}$ indicates that these functions are to be evaluated at $\underline{r}_{1}$. We decompose the integrations in the definition of $n_{0}^{(2)}\left(\underline{u}_{1}^{(2)}-\underline{u}_{2}^{(2)}\right)$ as follows

$$
\mathrm{n}_{0}^{(2)}\left(\underline{u}_{2}^{(2)}-\underline{u}_{1}^{(2)}\right)=\iint_{\underline{\gamma} \cdot \sigma>0} d \underline{v}_{1} d \underline{v}_{2} f_{0}^{(2)}\left(\underline{v}_{2}-\underline{v}_{1}\right)+\iint_{\gamma \cdot \sigma\langle 0} d \underline{v}_{1} d \underline{v}_{2} f_{0}^{(2)}\left(\underline{v}_{2}-\underline{v}_{1}\right)
$$


and then use the expression for $f_{0}^{(2)}$ in Eq. (2.99) to obtain

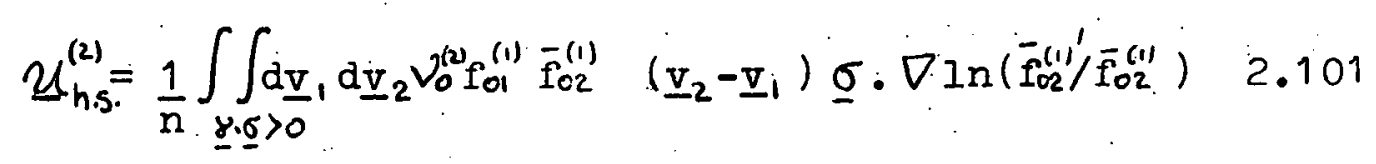

The effect of the collision is to change the direction of $\underline{\gamma}$, while leaving its magnitude constant. Using the identities

$$
\begin{aligned}
& \underline{\sigma}_{2}-\underline{\sigma}_{1}=2(\mathrm{k} \cdot \mathrm{T} / \mathrm{m})^{1 / 2} \underline{\underline{\gamma}} \quad \mathrm{C}_{2}^{2}-\mathrm{C}_{1}^{2}=(4 \mathrm{k} \mathrm{T} / \mathrm{m}) \underline{\Gamma} \cdot \underline{\gamma} \\
& \underline{W}_{2}^{\prime}-\underline{W}_{2}=\sqrt{2} \underline{\gamma} \cdot \underline{\hat{\sigma}} \underline{\hat{\sigma}} \quad W_{2}^{\prime 2}-W_{2}^{\prime 2}=-4 \underline{\gamma} \cdot \hat{\sigma} \hat{\sigma} \hat{\underline{\sigma}} \cdot \underline{\underline{\Gamma}} \\
& \underline{\Gamma}=1 / \sqrt{2}\left(\underline{w}_{2}+\underline{w}_{1}\right)
\end{aligned}
$$

and the change of variables $\left(\underline{v}_{2}, \underline{\nabla}_{1}\right) \rightarrow(\underline{\gamma}, \underline{\Gamma})$. we can further sim-, plify Eq. (2.101) to

$$
\underline{u}_{h, s_{0}}^{(2)}=-4 \hat{\hat{\sigma} \hat{\sigma}}: \nabla \underline{u} \iint d \underline{\gamma} d \underline{\underline{\sigma}} \cdot \underline{\gamma} \underline{\underline{\gamma}} e^{-\left(\Gamma^{2}+\gamma^{2}\right)}
$$

The integration over $\underline{\Gamma}$ is trivial. In the integration over $\underline{\gamma}$. It is convenient to use spherical coordinates. Let $\theta$ and $\varphi$ be the polar angles of $\underline{\gamma}$ with respect to $\sigma$, then

$$
\underline{u}_{h . s .}^{(2)}=(3 / 8) \underline{\hat{\sigma}} \hat{\sigma}: \nabla \underline{u} \underline{\sigma} \cdot \nu_{0}^{(z)} \int_{0}^{1} d y\left((3 y-1) \underline{\sigma} \underline{\sigma}+\left(1-y^{2}\right) \underline{U}\right)
$$

from which we find that

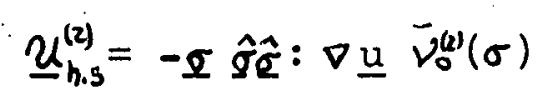

In the same way, from the definition of correlated temperatures and Eq. (2.99) we find that

$$
\mathrm{T}_{2}^{(2)}-\mathrm{T}_{1}^{(2)}=\frac{2}{3} \underline{\sigma} \cdot \nabla \mathrm{T}
$$

l'he results in Eqs. $(2.102,2.103)$ can now be used to choose the solenoidal part of $\underline{u}^{(2)}$ and $\underline{\underline{\tau}}^{(2)}$. From Eq. (2.102), the 
irrotational part of $\underline{\mathcal{u}}^{(2)}$ at $r=\sigma$ is

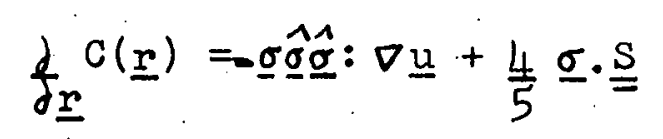

Subtracting this equation from $\mathcal{U}_{h \cdot s}^{(2)}$ we obtain

$$
\partial_{\underline{\underline{x}}} x \underline{D}(\underline{r})=-\frac{4}{5} \underline{\sigma} \cdot \underline{\underline{S}}
$$

Ihe vector

$$
\partial_{\underline{\underline{r}}} x \underline{D}(\underline{\underline{r}})=-\frac{\hat{\hat{r}}}{r^{2}}\left(g_{1}(r) \nabla \cdot \underline{u}+h_{3}(r) \underline{\underline{\hat{r}}} \underline{\underline{\underline{r}}}: \stackrel{\underline{s}}{=}\right)-\frac{\partial}{\partial_{\underline{r}}} C(\underline{r}) \quad-2.105
$$

is a difference of two particular solutions of the differential equation obeyed by $\underline{\mathcal{U}}^{(2)}$, and $i t$ is chosen to represent the solenoidal part of $\underline{U}^{(2)}$ for any potential because for hard spheres at $r=\sigma$ it reduces to Eq. (2.104). Adding Eqs. (2.91, $2.105)$, the general expression implied for $\underline{u}^{(i)}$ is

$$
\mathcal{U}^{(2)}=-\frac{\hat{\underline{r}}}{r^{2}}\left(g_{1}(r) \nabla \cdot \underline{u}+h_{3}(r) \underline{\underline{r}} \underline{\underline{r}}: \underline{\underline{S}}\right)
$$

where $h_{3}(r)=\int d x x^{2} h(x)$

This expression for $\underline{\mathcal{U}}^{(2)}$ was previously derived by Green and Hoffman (25) as a particular solution of Eq. (2.81). Similarly, from the definition of $\underline{\underline{\tau}}^{(2)}$, by using the same manipulations employed to derive Eq. (2.102), we obtain for $\underline{\tau}^{(2)}$ at $\underline{r}=\underline{\sigma}$

$$
\underline{\underline{\tau}}_{h \cdot s}^{(2)}=-\nu_{0}^{(2)}(\sigma) \frac{k}{m} \hat{\sigma} \underline{\underline{\sigma}} \underline{\sigma} \cdot \nabla T
$$

and

$$
\frac{\partial}{\partial \underline{\underline{r}}} \underline{\underline{r}}(\underline{\underline{r}})=-\frac{k}{m} \nu_{0}^{(2)}(\sigma)\left(\underline{\hat{\sigma} \underline{\sigma} \sigma} \cdot \nabla T-\frac{2 \sigma \sigma}{5} \cdot \nabla T \underline{\mathrm{U}}-\frac{2}{15} \underline{\sigma} \nabla T\right)
$$


which implies that, at $r=\sigma$

$$
\frac{\partial}{\partial \underline{\underline{r}}} x \underline{D}(\underline{\underline{r}})=-\frac{2 k}{5 m} \nu_{o}^{(2 \prime}(\sigma)\left(\underline{U} \sigma \cdot \nabla T+\frac{1}{3} \underline{\sigma} \nabla T\right) \quad 2.108
$$

As for the solenoidal part of $\mathcal{U}^{(2)}$, we assume that for a general potential

了,

$$
f_{\underline{r}} \dot{x} \underline{D}(\underline{r})=-\frac{k}{m r^{3}} g_{3}(r) \underline{r} \nabla T-\frac{k}{m r^{5}} h_{3}(r) \underline{r} \underline{r} \underline{r} . \nabla T-\frac{\partial}{\partial \underline{\underline{c}}}(\underline{r})
$$

where, $g_{3}(r)=\int d x x^{2} V_{0}^{(2)} \ln \nu_{0}^{(2)}$

Adding this equation to the general irrotational part of $\underline{\underline{\tau}}^{(2)}$ given by Eq. (2.95) we obtain

$$
\underline{\underline{\tau}}^{(2)}=-\frac{k}{m r^{3}} g_{3}(r) \underline{r} \nabla T+\frac{k}{m r^{5}} h_{3}(r) \underline{r} \underline{r} \underline{r} \cdot \nabla T \quad 2.109
$$

It is easily verified that this is a particular solution of Eq. (2.42) which is analogous to the correlated velocity function in Eq. (2.106). 
3. THE VISCOSITY AND THERMAL CONDUCTIVITY SECOND VIRIAL COEFFICIENTS

\subsection{The Kinetic Equation of a Moderately Dense Gas. Enskog's Equation from the BBGKY. Hierarchy}

In the low density limit the ratio $\sigma / \ell \approx \mathrm{r} . \nabla$ is very small, of the order of $10^{-3}$, and one would be justiified in treating $f_{0}^{(2)}$ as it appears in the first BBGKY equation as being a function of $\underline{r}_{1}$ only. In a linear theory, the spatial inhomogeneity of a dense gas can be taken into account by Taylor expanding the macroscopic spatial dependence of $f_{0}^{(2)}$ about $\underline{r}_{1}$ and neglecting all terms which are not linear in the macroscopic gradients, $i . e$. all terms of order $(\sigma / \ell)^{n}, n \geqslant 2$. If we consider only correlations from two body colitions, the resulting kinetic equation can then be written as a modified Boltzmann equation containing a corrective term due to collisional transfer of momentum and energy between the molecular centers of mass of the colliding molecules. This corroctive term alone gives density dependence to the transport coefficients ( 31 ) (the collisional contribution).

In the present kinetic theory of dense gases, beside the corrections just mentioned, we will introduce corrections due to correlations in the macroscopic parameters. In the case of multi-body collisions, corrections also arise when the correlations between colliding molecules which 
originated in previous collisions are taken into account. Correlations due to multiple collisions are not considered in the present dissertation.

After decomposing $f^{(2)}$ and $f^{(3)}$ as in Eq. (2.18) and with Eq. $(2.17)$ the consistency condition on $f_{0}^{(2)}$, Green and Hoffman. (25) were able to derive a self-consistent generalization of Boltzmann's equation for a dense gas. Their kinetic equation is of limited practical importance since it is difficult to solve. Hence the need to introduce into the theory an approximation which can simplify the problem arises. The hope is that the mathematical simplicity thus gained will not be offset by errors implicit in the new kinetic equation to represent the evolution of the state of the system.

As discussed in section 2.1, the decomposition of $f^{(2)}$ was made in such a way that $\mathrm{f}_{0}^{(2)}$ alone would contribute to the equations of change. As a result the condition in Eq. (2.74) was imposed on $f_{c}^{(n)}$. The first two terms in the integrand of Eq. (2.74) represent an implicit time derivative of $f_{c}^{(n)}$ along a trajectory traced by a cluster of n-molecules with respect to the relative position and velocity coordinates. We assume that $f_{0}^{(n)}$ is chosen so that $f_{C}^{(n)}$ is constant along these trajectories. For $n=2$ then $K_{0}^{(2)} f_{C}^{(2)}=0$

$$
\left(\underline{v}_{12} \cdot \frac{\partial}{\partial \underline{r}_{12}}-O_{12}-O_{21}\right)=K_{0}^{(2)} ; \quad K_{0}^{(2)} f_{e}^{(2)}=0 \quad 3.1
$$

This equation can be used exactly to relate $f_{c}^{(2)}$ in pre-colli- 
sion and post-collision configurations on the collision sphere at $r=\sigma$.

In the linearized form of the first BBGKY oquation

$$
D^{(1)} f_{0}^{(1)}-\int d \underline{x}_{2} O_{12} f_{0}^{(2)}=\int d \underline{x}_{2} O_{12} f_{c}^{(2)}
$$

we substitute Eq. (3.1) into its r.h.s. and transform the resulting integral into a surface integral by using Gauss's theorem

$$
D^{(1)} f_{0}^{(1)}-\int d \underline{x}_{2} O_{12} f_{0}^{(2)}=\int d \underline{\underline{v}}_{2} \int d \Sigma \underline{\hat{k}} \cdot \underline{\underline{v}}_{12} f_{c}^{(2)}
$$

Here $\hat{k}$ is a unit vector normal to the surface $\Sigma$ of an arbitrary sphere of radius $R$, with $R$ exceeding the range of the potential. Trajectories on this surface will all be nonbounded. From the solution of Eq. (3.1) we obtain

$$
f_{c}^{(2)}(t=0)=f_{c}^{(2)}\left(t=t_{c}\right)
$$

with $t_{c}$ representing the duration of a collision. We then decompose the surface $\Sigma$ into a pre and a post-collision hemisphere and rewrite the r.h.s. of Eq. (3.2) as

$$
\begin{aligned}
r \cdot h_{0} s_{0}=J_{B}^{(2)}(\Phi)= & \iint \underline{\hat{k}} \cdot \underline{\underline{v}}_{12} f_{c}^{(2)}\left(t_{c}\right) d \underline{\underline{v}}_{2} d \Sigma+ \\
& \iint \underline{\hat{k}} \cdot \underline{\underline{v}}_{12} f_{c}^{(2)}(t=0) d \underline{\underline{v}}_{2} d \Sigma
\end{aligned}
$$

On a nonbounded pre-collision trajectory $f_{c}^{(2)}$ can be approximated by the linearized molecular chaos assumption

$$
f_{c}^{(2)}(t=0)=f_{01}^{(1)} f_{02}^{(1)}\left(\Phi\left(v_{1}\right)+\Phi\left(v_{2}\right)\right)
$$

From Eq. (3.1) then 


$$
\begin{aligned}
& f^{(2)}\left(t=t_{c}\right)=f^{(1)^{\prime}} f^{(1)^{\prime}}\left(1+\Phi\left(v_{1}^{\prime}\right)+\Phi\left(v_{2}^{\prime}\right)\right) \\
& f_{c}^{(2)}\left(t=t_{c}\right)=f_{01}^{(1)^{\prime}} f_{02}^{(1)^{\prime}}\left(\Phi\left(v_{1}^{\prime}\right)+\Phi\left(v_{2}^{\prime}\right)\right) .
\end{aligned}
$$

In the second term of Eq. (3.3) we replace $\underline{\hat{k}}$ with $-\underline{\hat{k}}$, and then substitute Eqs. $(3.3,3.4,3.5)$ into Eq. (3.2) to obtain a generalized Boltzmann equation for a dense gas.

$$
\begin{aligned}
& D^{(1)} f_{0}^{(1)}-J^{(2)}\left(f_{0}^{(2)}\right)=J_{B}^{(2)}(\Phi) \\
& J^{(2)}() \equiv \int d \underline{X}_{2} O_{12}()
\end{aligned}
$$

$J_{B}^{(2)}(\Phi)$ is Boltzmann's collision operator. Assumptions that we will make concerning $f_{c}^{(2)}$ on bounded trajectories will not affect the generality of the above kinetic equation since Boltzmann's collision integral is ovaluated over a surface that exceeds the range of the potential, on which of course there are no bound states and $\nu_{0}^{(2)}=1$.

In the linearization of Eq. (3.6), frequent use of certain relationships involving the second virial coefficient $B(T)$ and its temperature derivatives is made. The identities

$$
\begin{aligned}
& B(T)=\frac{1}{2} \int d \underline{r}\left(1-\nu_{0}^{(2)}\right)=\frac{1}{6} \int d \underline{r} \frac{\partial \nu_{0}^{(2)}}{\partial r} \\
& 2 T \frac{\partial}{\partial T} B(T)=2 T \dot{B}(T)=\int d \underline{r} \nu_{0}^{(2)} \ln \nu_{0}^{(2)} \\
& B(T)+T \dot{B}(T)=-\frac{1}{6} \int d \underline{r} \ln \nu_{0}^{(2)} \frac{\partial}{\partial r} P_{0}^{(2)} \\
& 4 T \dot{B}(T)+2 T^{2} \ddot{B}(T)=-\int d \underline{r} P_{0}^{(2)}\left(\operatorname{In} \nu_{0}^{(2)}\right)^{2} \\
& \frac{1}{n C_{V}}\left(\frac{\partial P}{\partial T}\right)_{v}=\frac{2}{3}(1+n Z(T)+\ldots)
\end{aligned}
$$




$$
Z(T)=B(T)+\frac{7}{3} T \dot{B}(T)+\frac{2}{3} T^{2} \ddot{B}(T)
$$

will be extensively used in this chapter. By substitution of the Taylor expansion of $f_{0}^{(2)}$ about $\underline{r}_{1}$.

$$
\begin{aligned}
& f_{0}^{(2)}=\bar{f}_{0}^{(2)}\left(1+\underline{r} \cdot \nabla \ln \underline{f}_{0}^{(2)}+\frac{1}{2} \underline{r} \cdot \nabla \ln \nu_{0}^{(2)}\right) \\
& \bar{f}_{0}^{(2)}=f_{01}^{(1)} \bar{f}_{02}^{(1)} \nu_{0}^{(2)}
\end{aligned}
$$

into Eq. (3.6), we obtain the inhomogeneous part of Boltzmann's equation plus a corrective term

$$
\begin{aligned}
J_{B}^{(2)}(\Phi)= & \left(\frac{\partial}{\partial t}+\underline{v}_{i} \frac{\partial}{\partial \underline{\underline{r}}}\right) f_{0}^{(1)}-J^{(2)}\left(\bar{f}_{0}^{(2)}\left(\underline{r} \cdot \nabla \ln \bar{f}_{02}^{(1)}+\frac{1}{2} \underline{r} \cdot \nabla \ln \nabla_{0}^{(2)}\right)\right)- \\
& J^{(2)}\left(\bar{f}_{0}^{(2)} p_{1}^{(2)}(W, r)\right) \\
= & f_{01}^{(1)}\left(2 \underline{W}: \underline{\underline{S}}+\left(\frac{5}{2}-W^{2}\right) \underline{C} \cdot \nabla \ln T+\frac{2}{3} n Z(T)\left(\underline{3}-W^{2}\right) \nabla \cdot \underline{u}\right) \\
& -J^{(2)}\left(\bar{f}_{0}^{(2)} p_{1}^{(2)}(W, r)\right)
\end{aligned}
$$

where $W=\left(m / 2 k^{\prime} \mathrm{T}\right)^{\frac{1}{2}} \underline{C}$

$$
\begin{aligned}
& p_{k}^{(n)}(W, r)=2 \underline{W}_{k} \cdot\left[W_{k}\right]^{(n)}+2 \underline{W}_{k} \underline{W}_{k}:\left[\underline{W}_{x} \underline{W}_{k}\right]^{(n)}-\left[W_{k}^{2}\right]^{(n)}
\end{aligned}
$$

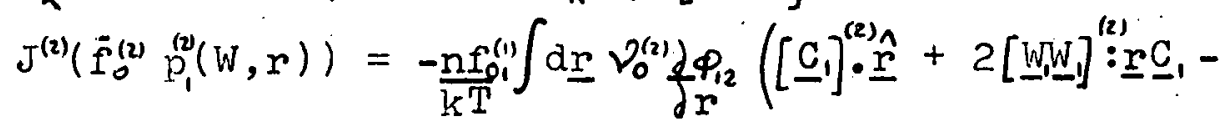

$$
\begin{aligned}
& \frac{m}{k T}\left(\underline{C}_{1} \cdot \underline{\hat{\underline{x}}}\left[\underline{C}_{1}\right]^{(2)} \cdot \underline{C}+\underline{C}, \underline{G}:\left[W_{W}\right]^{(2)} \underline{\underline{r}} \cdot \underline{C}_{i}-\frac{1}{2}\left[C_{i}^{2}\right]^{(2)}\right)
\end{aligned}
$$

For the hard spheres molecular model the corrective term reduces to

$$
\begin{aligned}
-J\left(\bar{f}_{0}^{(2)} p_{1}^{(2)}(W, r)\right)= & \left(\frac{4}{9} \pi \sigma^{3}\right)\left(W^{2}-\frac{3}{2}\right) \nabla \cdot \underline{\underline{u}}+2\left(\frac{\left.4 \pi \sigma^{3}\right)}{15} \underline{W} \underline{\underline{S}}+\right. \\
& \frac{\left(6 \pi \sigma^{3}\right)}{15}\left(\frac{3}{2}-W^{2}\right) \underline{c} \cdot \nabla \ln T
\end{aligned}
$$


This result, substituted into Eq. (3.12) yields Enskog's equation for the case $V_{0}^{(2)}=1$

$$
\begin{aligned}
& J_{\theta}^{(2)}(\Phi)=\mathrm{f}_{01}^{(1)}\left(2\left(1+n \mathrm{~F}_{\eta}\right) \underline{W W}: \underline{\underline{S}}+\left(1+n F_{\lambda}\right)\left(W^{2}-5 / 2\right) \underline{\mathrm{C}} \cdot \nabla \operatorname{lnT}+\right. \\
& \left.\mathrm{n}\left(\frac{2}{3} \mathrm{Z}(\mathrm{T})+\mathrm{F}_{k}\right)\left(3 / 2-\mathrm{W}^{2}\right) \nabla \cdot \underline{u}\right) \quad 3.13 \\
& F_{\eta}=\frac{4 \pi \sigma^{3}}{15}, \quad F_{\lambda}=\frac{2 \pi \sigma^{3}}{5}, \quad F_{k}=\frac{-2 Z(T)}{3}
\end{aligned}
$$

Had we chosen $f_{0}^{(2)}=f_{01}^{(11} f_{02}^{(1)} \varphi_{0}^{(2)}$ everywhere, then all correlated parameters in section 2.6 vanish and Eq. (3.12) would reduce to the original Boltzmann's equation. It is the correlation in the velooities that gives a density dependence to the transport coefficients.

In section 3.3 we will decompose the expression for the flux of momentum as in E'. (2.19), and then, after: evaluating the kinetic and collisional contributions, we will compare Eq. (2.19) with its equivalent phenomenological equation to obtain $\eta$ the coefficient of shear viscosity. The function $f_{c}^{(1)}$ and therefore $\underline{\underline{P}}_{k}\left(f_{c}^{(1)}\right)$ is correct to terms linear in the density while $\stackrel{P}{=}_{\psi}$ is correct to $n^{2}$ terms, hence the $n^{2} \underline{S}$ terms will bo discarded. The orthogonality conditions on $\mathrm{f}^{\text {(i) }}$ can be used to show that there is no kinetic contribution to the coefficient of bulk viscosity $k$; implying that $K$ can be evaluated correctly to $n^{2}$ terms (since $\underline{\underline{P}}_{\varphi}$ is correct to terms of this order in n.). These same arguments can be used to show that $\lambda$, the thermal conductivity coefficient, can be correctly evaluated only to $n$ ' terms. 
In the following discussion, for reasons just explained we will neglect terms contafning $\underline{T}$ or $\underline{\underline{S}}$ whose order in the density is greater than one and all $n^{m} \nabla$. $m>2$. The disappearance of the coefficient of bulk viscosity from Eq. (3.13) arises from truncation of the density expansion of $\nu_{0}^{(\nu)}$ after the first term.

The r.h.s. of Eq. (3.13) is to be regarded as the inhomogeneity of the generalized Boltzmann's equation. The corrective terms $F_{\eta}$ and $F_{A}$ in general are functions of temperature and are obtained by equating Eq. (3.12) to Eq. (3.13) and then by taking the velocity moments of the resulting identity. We find in this way a set of equations involving $F_{\lambda}, F_{\eta}$ and $F_{k}$ :

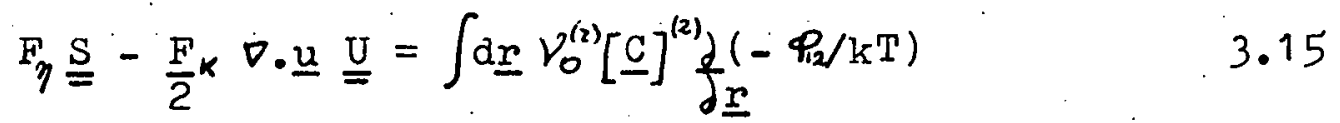

$$
\begin{aligned}
& F_{\lambda} \nabla \operatorname{InT}=\frac{2 m}{5 k T} \int d \underline{\underline{k}} \nu_{0}^{(2)}\left([C \underline{C}]^{(2)}+\frac{1}{2}\left[C^{2}\right]^{(2)} \underline{\underline{U}}\right) \cdot \frac{\partial}{\partial \underline{\underline{r}}}\left(-\varphi_{Q} / k T\right)
\end{aligned}
$$

The expressions for $\underline{\mathcal{U}}^{(2)}$ and $\underline{\zeta}^{(2)}$ in Eqs. $(2.93,2.96)$ together with the 1dentities in Eqs. $(3.7,3.8,3.9,3.10,3.11)$ yields

$$
\begin{aligned}
& F_{\eta}=\frac{2}{5}(B(T)+T \dot{B}(T)) \\
& F_{\lambda}=\frac{3}{5}\left(B(T)-\frac{5 T}{9}(T)-\frac{7 T^{2}}{9} \ddot{B}(T)\right) \\
& F_{k}=-\frac{2}{3} Z(T)
\end{aligned}
$$

Fior the hard sphere model Eqs. $(3.17,3.18,3.19)$ reduce to 
Eq. (3.16).

3.2 The Perturbation Solution of Boltzmann's Equation

Boltzmann collision operator $J_{B}^{(2)}$ is self adjoint in the sense that it obeys the following identity:

$$
\left\langle\Psi_{k}, J_{B}^{(2)}(\Phi)\right\rangle=\left\langle J_{B}^{(2)}\left(\Psi_{k}\right), \Phi\right\rangle
$$

where $\left\langle\bar{\Psi}_{k}, J_{B}^{(2)}(\Phi)\right\rangle=\int d \underline{v} \Psi_{k} J_{B}^{(2)}(\Phi)$

If $\Psi_{k}$ is any summational invariant $1, \underline{v}, v^{2}$ or a linear combination of sumational invariants, then

$$
\left\langle J_{B}^{(2)}\left(\Psi_{k}\right), \Phi\right\rangle=0
$$

Chapman and Cowling, in their famous book, (3) have shown that the Fredholm theorem for an integral equation like (3.13) has a solution if the inhomogeneous part, the r.h.s. of Eq. (3.13), is orthogonal to $1, \underline{v}, v^{2}$, the solutions of the homogeneous equation. Hence

$$
\begin{aligned}
& \int d \underline{v}\left(\Psi_{k}\right)_{k=1}^{3}\left(1+n F_{\eta}\right)\left(\underline{W} \underline{W}-\frac{1}{3} W^{2} \underline{U}\right) f_{0}^{(1)}=0 \\
& \int d \underline{v}\left(\Psi_{k}\right)_{k=1}^{3}\left(\left(W^{2}-\frac{5}{2}\right)+n F_{\lambda}\right) \underline{C} f_{0}^{(1)}=0
\end{aligned}
$$

for the othogonality conditions

$$
\int d \underline{v}\left(\Psi_{k}\right)_{k=1}^{3}\left(D^{(1)} f_{0}^{(1)}-\int d \underline{X}_{2} \ddot{O}_{12} f_{0}^{(21}\right)=0
$$

to be satisfied. Condition (3.20) is always satisfied independently of the form of $F_{\eta}$. In fact, for $\left(\bar{\Psi}_{2}\right)=\underline{v}$ the 
integrand is odd in $\underline{W}$ and simple symmetry arguments can be used to show that the integral reduces to zero. For $\Psi_{1}=1$, $\Psi_{3}=v^{2}$, the integral, upon integration over the orientation angles of $\underline{W}$, vanishes.

The Chapman and Enskog method of solution of the Boltzmann equation is discussed in many textbooks on nonequilibrium thermodynamics, details of the method can be found in references (32), (33) and (7). The method is based on an expansion of $f^{(1)}$ about its local equilibrium form $f_{0}^{(1)}$

$$
f^{(1)}=f_{0}^{\prime \prime \prime}(1+\Phi)
$$

To find $\Phi$, the perturbation coefficient, we must solve Eq. (3.13); the auxiliary conditions of orthogonality

$$
\int \mathrm{d} \underline{\mathrm{v}}\left(\Psi_{k}\right)_{k=1}^{3} \quad \mathrm{fo}^{\prime \prime \prime} \Phi=0
$$

will make the solution $\Phi(n, \underline{u}, T)$ of Eq. (3.13) unique. We define the following tensors:

$$
\begin{aligned}
& \underline{k}=f_{0}^{\prime \prime \prime}\left(1+n F_{\lambda}\right)\left(W^{5}-\frac{5}{2}\right) \underline{G} \\
& \underline{\mathscr{L}}=2 f_{0}^{\prime \prime \prime}\left(1+n F_{\eta}\right)\left(\underline{W} \underline{W}-\frac{1}{3} W^{2} \underline{U}\right)
\end{aligned}
$$

In this notation Eq. (3.13) becomes

$$
J_{B}^{(2)}(\Phi)=\underline{\underline{x}}: \underline{s}+\underline{\underline{k}} \cdot \nabla \ln \mathrm{T}
$$

We have already seen that $\Phi_{n}=\left(1, \underline{v}, v^{2}\right)$ are homogeneous solutions of this equation. Since $J_{B}^{(2)}(\Phi)$ is linear in $\Phi$, 
the particular solution. $\Phi_{p}$ of Eq. (3.26) must be linear in S and $\nabla$ InT. Let the scalar function $\Phi$ be given by

$$
\Phi=\Phi_{h}+\Phi_{P}=\sum_{i=1}^{3} c_{i} \psi_{i}+\underline{A} \cdot \nabla \ln \mathrm{T}+\underline{\underline{B}}: \underline{\underline{S}}
$$

The five constant $c_{i}$ will be determined by the five conditions of orthogunality. From Eq. $(3.23)$ we find that $c_{1}=c_{5}=0$; the constants $c_{2}, c_{3}, c_{4}$ will be incorporated into 4 to transform Eq. (3.27) to

$$
\Phi=\underline{A} \cdot \nabla \ln T+\underline{\underline{B}}: \underline{\underline{S}}
$$

From the linearity of Boltzmann operator $\mathrm{J}_{B}^{(2)}$ we obtain

$$
J_{B}^{(2)}(\Phi)=J_{B}^{(2)}(\underline{A}) \cdot \nabla \ln T+J_{B}^{(2)}(\underline{\underline{B}}): \underline{\underline{S}}
$$

comparing this equation with Eq. (3.26) leads to the following integral equations for $\underline{A}$ and $\stackrel{B}{=}$

$$
\begin{aligned}
& \stackrel{\mathscr{E}}{=}=J_{B}^{(2)} \stackrel{(\underline{B})}{=} \\
& \underline{k}=J_{B}^{(2)} \underline{(A)}
\end{aligned}
$$

Since $J_{B}^{(2)}$ is a scalar operator, it follows from iqs. 13.24 , $3.25,3.30,3.31$ ) that $\stackrel{Q}{=}$ must be a traceless, symmetric, second rank tensor and $\mathcal{A}$ a vector. The form for $\underline{\mathbb{B}}$ and $\mathcal{A}$ given below,

$$
\begin{aligned}
& \underline{\underline{B}}=B(W) \quad\left(\underline{W} \underline{W}-\frac{1}{3} W^{2} \underline{U}\right) \\
& \underline{A}=\mathcal{A}(W) \underline{W}
\end{aligned}
$$


is a consequence of Eqs. $(3.30,3.31)$, the two vector equations in $\underline{w}$.

The coefficients of proportionality $B(W), A(W)$, are expanded in terms of Sonine polynomials

$$
\begin{aligned}
& A(w)=\sum_{k=0}^{\infty} a_{k} s_{3 / 2}^{(k)}\left(w^{2}\right)=a_{0}+a_{1}\left(5 / 2-w^{2}\right)+\ldots 3.34 \\
& B(w)=\sum_{k=0}^{\infty} b_{k} S_{5 / 2}^{(k)}\left(w^{2}\right)=b_{0}+\ldots \ldots
\end{aligned}
$$

Sonine polynomials are chosen as basis functions for the expansion of $A$ and $B$ as a matter of convenience. The orthogonality relation between the polynomials $\mathrm{s}_{3 / 2}^{(i)}(i=0,1,2 \ldots \ldots)$ for $\mathcal{A}$ and $S_{5 / 2}^{(i)}(i=0,1,2 \ldots .$.$) for B$ guarantee that only the $S_{3 / 2}^{(1)}$ contribution to the kinetic part of the energy flux and the $S_{S / 2}^{(0)}$ contribution to the kinetic part of the dissipative momentum flux will be nonzero. The simplest approximation then results from retention of only these nonzero contributions in the solutions :

$$
\begin{aligned}
& \mathcal{A}(w) \approx a_{1}\left(5 / 2-w^{2}\right) \\
& B(w) \approx b 0
\end{aligned}
$$

To find $a_{1}$, bo we equate Eqs. $(3.26,3.29)$

$$
\underline{\mathscr{L}}: \underline{\underline{S}}+\underline{\underline{k}} \cdot \nabla \ln \mathrm{T}=J_{\mathrm{B}}^{(2)}(\underline{\underline{A}}) \cdot \nabla \ln \mathrm{T}+\mathrm{J}_{\mathrm{B}}^{(2)}(\underline{\underline{Q}}): \underline{\underline{S}} \quad 3.37
$$

Note that the zeroth and first moments of Eqs. (3.26, 3.29) give the first two equations of change as expected since $m$ and rav are collision invariants in both the dilute and dense gas case and lead to the trivial result $0=0$. On Eq. (3.37) we 
operate on both sides with $\int \mathrm{d} W \underline{W} \underline{W}()$. From symmetry considerations the $\nabla$ InT term vanish and an expression for $b_{0}$ is found. The opposite effect will be obtained by operating on the same identity with $\int \mathrm{d} W \underline{W} \underline{W}()$. W o obtain

$$
\begin{aligned}
& \left(\langle\underline{W} \underline{W}, \underline{\mathscr{L}}\rangle-b_{0}\left\langle\underline{W} \underline{W}, J_{B}^{(2)}(\underline{W} \underline{W})\right\rangle\right): \underline{S}=0 \\
& \left(\langle\underline{W} \underline{W}, \underline{\underline{K}}\rangle-a_{1}\left\langle\underline{W} \underline{W}, J_{B}^{(2)}\left(\underline{C}\left(5 / 2-W^{2}\right)\right)\right\rangle\right) \cdot \nabla \ln T=0
\end{aligned}
$$

There are no difficulties in proving that

$$
\begin{aligned}
& \langle\underline{W W}, \underline{\underline{L}}\rangle=\mathrm{n} / 2(\mathrm{~m} / 2 \mathrm{k} T)^{3 / 2}\left(1+\mathrm{nF}_{\eta}(T)\right)(\underline{\underline{\underline{W}}}+\underline{\underline{\underline{\underline{W}}}+\underline{\underline{U}} \underline{\underline{U}})} \quad 3.40 \\
& \langle\underline{W W}, \underline{\underline{x}}\rangle=\mathrm{n} / 4(\mathrm{~m} / 2 \mathrm{kT})(1+\mathrm{nF}(\mathrm{T}))(\underline{\underline{\underline{\underline{w}}}}+\underline{\underline{\underline{\underline{w}}}}+\mathrm{UW}) \quad 3.41
\end{aligned}
$$

The integral

$$
\begin{aligned}
& \left\langle\underline{W} \underline{W}, J_{B}^{(2)}(\underline{W} \underline{W})\right\rangle=\int d \underline{W} \underline{W} \underline{W} \int d \underline{v}_{2} \int d \Sigma \underline{\hat{k}} \cdot \underline{v}_{-2} f_{01}^{(1)} f_{02}^{(1)}\left(\underline{w}_{1}^{\prime} \underline{w}_{1}^{\prime}+\right. \\
& \left.\underline{w}_{2}^{\prime} \underline{W}_{2}^{\prime}-\underline{w}_{1} \underline{w}_{1}-\underline{w}_{2} \underline{w}_{2}\right) \quad 3.42
\end{aligned}
$$

is, however, more difficult. This integration is simplified if we replace the integration over the area $\Sigma$ by an integration over the projection of $\Sigma$ on a plane normal th the direction of $v_{13 .}$. If the z-axis is made to coincide with the direction of $\underline{v}_{12}$ then $\underline{v}_{12} \cdot \underline{\hat{k}}=v_{12} \cos \theta$ and $\int \ldots . \hat{\underline{k}} \cdot \underline{\underline{v}}_{12} d \Sigma=. \int_{0}^{3 \pi} d \varphi \int_{0}^{\pi} d \theta v$ $\cos \theta \sin \theta$. From the geometry of the trajectory the impact parameter $b$ is equal to $r \sin \theta$ and hence $\int \ldots \hat{\underline{k}} \cdot \underline{v}_{12} d \Sigma=\ldots$ $\int_{0}^{2 \pi} d \theta \int_{0}^{\sigma} \cdots v_{12} b d b$. Eiq. (3.40) can be further simplified by the transformation of variables $\left(\underline{W}_{1}, \underline{W}_{2}\right) \rightarrow(\underline{\gamma}, \underline{\Gamma})$. We obtain 


$$
\begin{aligned}
& \left\langle\underline{W} \underline{\underline{W}}, J_{B}^{(2)}(\underline{W} \underline{W})\right\rangle=\left(m n^{2} 2^{1 / 2} / \pi^{2} k T\right) \iiint d b d \underline{\underline{p}} d \underline{\gamma} \int_{0}^{\sigma}\left(\underline{W}_{1}^{\prime} \underline{w}_{1}^{\prime}+\right. \\
& \underline{W}_{2}^{\prime} \underline{W}_{2}^{\prime}-\underline{W}_{1} \underline{W}_{1}-\underline{W}_{2} \underline{W}_{2} e^{-\left(P^{2}+\gamma^{\prime 2}\right)}
\end{aligned}
$$

Since $W^{2}$ is a sumational invariant (i,e. the kinetic energy') we can replace $J_{B}^{(2)}(\underline{W})$ with $J_{B}^{(2)}\left(\underline{W W}-1 / 3 W^{2} \underline{W}\right)$. There is only one fourth-rank isotropic tensor traceless on its last two indices

$$
\left\langle\underline{W W}, J_{B}^{(2)}\left(\underline{W W}-1 / 3 W^{2} \underline{\underline{U}}\right)\right\rangle=A(1 / 2(\underline{\underline{\underline{\underline{W}}}}+\underline{\underline{\underline{\underline{\underline{U}}}}})-1 / 3 \underline{\underline{U}})
$$

The coefficient of proportionality $A$ will be found by dotting 岸 into the above equation

$$
5 A=\underline{\underline{w}} \vdots\left\langle\underline{W}, J_{B}^{(2)}(\underline{W W}-1 / 3 W \underline{\underline{U}})\right\rangle
$$

After some lengthy but straightforward manipulations we find. that $A=\left(4 n^{2} m^{1 / k} \sigma^{2} \Omega^{(2,2) *}\right) /\left(2^{1 / 2 k T}\right)$, where the dimensionless. viscosity cross section, $\Omega^{(2,2)^{*}}$ is defined by

$$
\Omega^{(2,2)^{*}}=\int_{0}^{\infty} \int_{0}^{\infty} d \gamma^{\prime} d b b \gamma^{\prime \prime}\left(1-\cos ^{2} x\right) e^{-\gamma^{\prime 2}}
$$

here $x$ is the angle between $\gamma$ and $\underline{\gamma}^{\prime}$ (the scattering angle). An expression for $b_{0}$ can now be obtained by substituting Eqs. $(3.40,3.43)$ into Eq. $\left(3.3^{8}\right)$. Thus we find that

$$
b_{n}=(m / \pi k T)^{1 / 2} \frac{1.5\left(1+n F_{n}(T)\right)}{24 n \sigma^{2} \Omega^{(1,2) *}}
$$

The solution of Eq. (3.39) does not present any new problem, we find 


$$
a_{i}=-(2 / \pi)^{1 / 2} \frac{15\left(1+n F_{\lambda}(T)\right)}{32 n \sigma^{2} \Omega^{(2,2) *}}
$$

These two last results complete the perturbation solution and yield

$$
\begin{gathered}
\Phi=a_{1}(5 / 2-W) \underline{C} . \nabla \ln T+b_{0}\left(\underline{w}-1 / 3 w^{2} \underline{U}\right): \underline{S}: 3.46 \\
3.3 \text { The Viscosity Coefficient }
\end{gathered}
$$

Irving and Kirkwood (34) have derived general formulas for the flux of momentum and thermal energy. Their expressions will be used in this section to obtain an equation for the coefficient of shear viscosity. The kinetic and collisional contribution to the flux of momentum across a surface moving along with velocity $\underline{u}$, the gas streaming velocity, is given by $\underline{\underline{p}}_{k}$ : $\hat{\underline{n}}$ and $\underline{\underline{p}}_{\varphi} \cdot \underline{\hat{n}}$, $\underline{\hat{n}}$ is a unit vector normal to the surface just mentioned and

$$
\begin{aligned}
\underline{\underline{P}}_{k} & =\int d \underline{\mathrm{V}} \underline{\mathrm{C}} \underline{\mathrm{C}} \mathrm{f}^{(1)} \\
& =\int \mathrm{d} \underline{\mathrm{V}} \underline{\mathrm{C}} \underline{\mathrm{C}} f^{(1)}(1+\Phi)=n k \mathrm{\Phi} \underline{\underline{\mathrm{U}}}-\mathrm{nkTb_{0 }} \underline{\underline{S}}
\end{aligned}
$$

and

$$
\stackrel{P}{=} \Phi=-\frac{1}{2} \iiint d \underline{v}_{1} d \underline{r}_{2} d \underline{v}_{2} r \underline{\underline{r}} \underline{\underline{r}}\left(f^{(2)}-\frac{1}{2} \underline{r} \cdot \nabla f^{(2)}\right) \frac{\partial}{\partial r} \phi_{12} \quad 3.48
$$

Upon substitution of the previously defined expressions for $f^{(2)}, f_{0}^{(2)}, f_{c}^{(2)}$ and $\Phi$, see Eqs. $(2.18,2.55,3.5,3.46)$, into Eq. (3.48), P can be rewritten as the sum of four integrals. One of the integrands contains $\nabla f_{c}^{(2)}$, since $f_{C}^{(2)}$ is already first order in $\nabla$, this integral will be neglected. If we substitute Eq. (2.55) for $\mathrm{f}_{0}^{(2)}$ into the integrand containing res $\underline{\underline{r}} . \nabla f_{0}^{(2)}$, 
we will obtain integrands which are either odd in $\underline{r}$ or in $\underline{C}$, and therefore these integrals will all vanish. The two remaining integrals are:

$$
\begin{aligned}
& \stackrel{P}{=}_{\varphi}^{(1)}=-\frac{1}{2} \iiint d \underline{r} d \underline{v}_{1} d \underline{v}_{2} r \underline{\hat{r}} \underline{\hat{r}} f_{0}^{(2)} \frac{\partial}{\partial r} \phi_{12}=(p-n k T) \underline{U}=3.49
\end{aligned}
$$

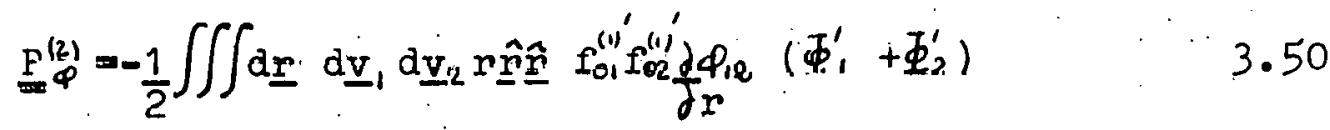

Eqs. $(3.47,3.49,3.50)$ show that $f_{0}^{(2)}$ does not contribute to the dissipative part of $\underline{\underline{p}}$ and that $\underline{\underline{P}}\left(f_{0}^{(1)}, f_{0}^{(2)}\right)=p \underline{\underline{U}}$ as required by condition 2.20. We substitute next Eq. (3.46) into the symmetric integrand of $\stackrel{P}{=}(2)$ and obtain

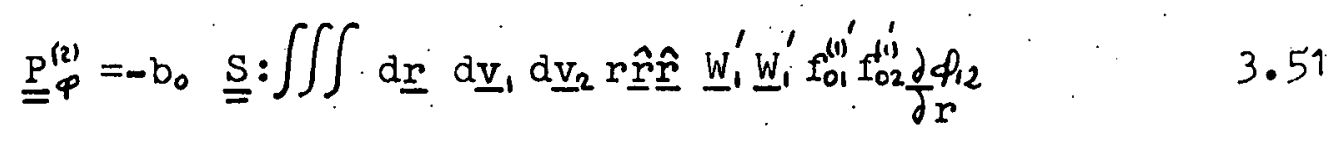

In section 3.2 we have discussed the transformation of this type of integral to scalar form. In this case this procedure yields

$$
\stackrel{P}{\rightarrow}(2)=-\frac{b_{0}}{15} I_{\eta} \stackrel{S}{=}
$$

The integral $I_{\eta}$ is defined by

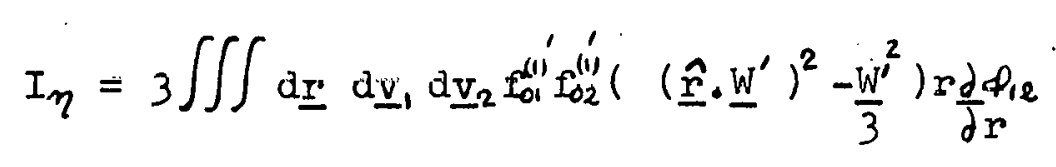

Comparing the analytical expression for the pressure tensor $\stackrel{P}{=}=\underline{P}_{k}+\underline{P}_{p}$ obtained from Eqs. $(3.47,3.49,3.53)$,

$$
\underline{\underline{p}}=p \underline{\underline{U}}-\left(n k T b_{0}+(1 / 15) b_{0} I_{\eta}\right) \underline{\underline{S}} \text {, }
$$

with the equivalent phenomenological equation, we obtain the following expression for the coetficient of shear viscosity 


$$
\eta=\eta_{0}\left(1+\left(F_{\eta}+\frac{4 \pi^{1 / 2}}{15 k T} I_{\eta}\right) n+\ldots\right)
$$

The coefficient of shear viscosity $\eta$, can be written to linear terms in the density in the form

$$
\eta=\eta_{0}\left(1+n \sigma^{3} \beta_{\eta}^{*} \ldots\right)
$$

$\eta_{0}$ is the usual dilute gas viscosity coefficient. From Eqs. $(3.55,3.56)$ we can solve for $B_{\eta}^{*}\left(T^{*}\right)$ and obtain

$$
B_{\eta}^{*}\left(T^{*}\right)=\frac{1}{\sigma^{3}} \cdot\left(F_{\eta}+\frac{4 \pi^{1 / 2}}{15 k^{T}} I_{\eta}\right)
$$

where $B_{\eta}^{*}=3 B_{\eta} / 2 \pi \sigma^{3}$. As we have mentioned in chapter 1 . a density expansion of the transport coefficients does not exist and higher order terms involve also a logarithmic. dependence on the density.

3.4 The Thermal Conductivity Coefficient

As in the case of the momentum flux, the heat flux vector $\mathrm{q}$ can be decomposed into a kinetic part

$$
\underline{q}_{k}=\int d \underline{v} 1 / 2 \mathrm{mC}^{2} \underline{\underline{C}}{ }^{(1)}=5 / 4 \mathrm{nka}, \quad(2 \mathrm{kT} / \mathrm{m})^{1 / 2} \nabla \mathrm{T} \quad 3.58
$$

and a potential contribution

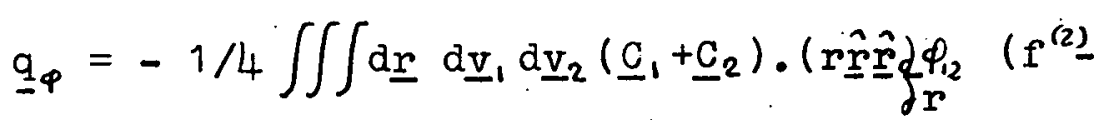

$$
\begin{aligned}
& \left.\left.1 / 2 \underline{\underline{r}} \cdot \nabla \mathrm{f}^{(2)}\right)-\phi_{12} \mathrm{f}^{(2)} \underline{\underline{U}}\right)
\end{aligned}
$$

Neglecting integrands odd in either $\underline{\mathrm{C}}, \underline{r}$, or nonlinear $\nabla, \underline{q}$ reduces to a sum of two integrals containing only $f_{c}^{(2)}$, 
implying that $\underline{q}_{x}\left(f_{0}^{(1)}\right)=q_{\varphi}\left(f_{0}^{(2)}\right)=0$. We have in this way verified the last consistency condition on $f_{0}^{(2)}$ expressed in Eq. (2.19).

From Eq. (3.59), after replacing $\left(\underline{v}_{1}, \underline{v}_{2}\right)$ with the corresponding reduced velocities $\left(\underline{\boldsymbol{\gamma}}, \underline{\Gamma_{2}}\right)$ and performing the

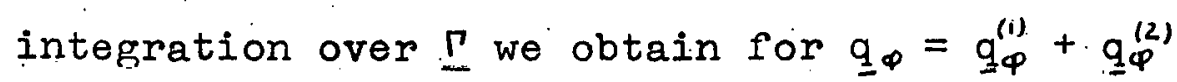

$$
\begin{aligned}
& \underline{q}_{\phi}^{(1)}=(2 \mathrm{kT} / \mathrm{m})^{\frac{1}{2}}\left(\mathrm{n}^{2} a_{1} \nabla \operatorname{InT} / 12 \pi^{\frac{2}{2}}\right) I_{\lambda}^{(1)} \\
& 3.60 \\
& \underline{q}_{\varphi}^{(2)}=\left(2 k^{r} / m\right)^{\frac{1}{2}}\left(5 n^{2} a_{1} \nabla \ln T / 24 \pi^{\frac{1}{2}}\right) I_{\lambda}^{(2)}
\end{aligned}
$$

where: $I_{\lambda}^{(1)}=\iint d \underline{r} \underline{d \gamma} r \frac{\partial}{\partial r} \Phi_{12}\left(5 / 4-1 / 2 \gamma^{\prime 2}-\left(\underline{\hat{r}} \cdot \underline{\gamma^{\prime \prime}}\right)^{2}\right) \theta^{-\gamma^{\prime 2}}$.

$$
I_{\lambda}^{(2)}=\iint d \underline{r} d \underline{\gamma} \Phi_{12}\left(\gamma^{\prime 2}-3 / 2\right) \theta^{-\gamma^{\prime 2}}
$$

To find $\lambda$ we compare the sum $\underline{q}_{\kappa}+\underline{q}_{\phi}$ with the phenomenological expression for $\underline{q}$,

$$
\underline{q}=-\lambda \nabla \mathrm{T}=-\lambda_{d}\left(1+n\left(F_{\lambda}+\left(1 / 15 \pi^{3 / 2} k T\right) I_{\lambda}\right)+\ldots\right) \nabla \mathrm{T} \quad 3.64
$$

where $I_{\lambda}=I_{\lambda}^{(1)}+5 / 2 I_{\lambda}^{(2)}$

If we let

$$
\lambda=\lambda_{0}\left(1+n \sigma^{3} \beta_{\lambda}^{*}+\ldots\right)
$$

then $B_{\lambda}^{*}=\frac{1}{\sigma^{3}}\left(F_{\lambda}+\left(1 / 15 \pi^{3 / 2} k^{\prime T}\right) I_{\lambda}\right)$

where $B_{\lambda}^{*}=3 B_{\lambda}^{*} / 2 \pi \sigma^{3}$; $\lambda_{0}$ is the dilute gas thermal conductivity coefficient. 
4. TRANSPORT COEFFICIENTS FOR THE HARD SPHERE AND SQUARE WELL MODELS

\subsection{Transport Coefficients for Hard Spheres}

In order to obtain the viscosity and thermal conductivity second virial coefficients for a gas of hard spheres we must solve the integrals $I_{\eta}$ and $I_{\lambda}$ in Eqs. $(3.53,3.62$, 3.63). These nine fold integrals can be readily reduced to six fold integrals by symmetrizing their respective integrands and changing the integration variables to $(\underline{\boldsymbol{y}}, \boldsymbol{\Gamma})$. The integration over. $\boldsymbol{T}$, the velocity of the center of mass, can then be performed immediately. It is convenient to decompose In into two parts as follows:

$$
\begin{aligned}
& I_{n}=3 I_{n}^{(1)}-I_{n}^{(2)}
\end{aligned}
$$

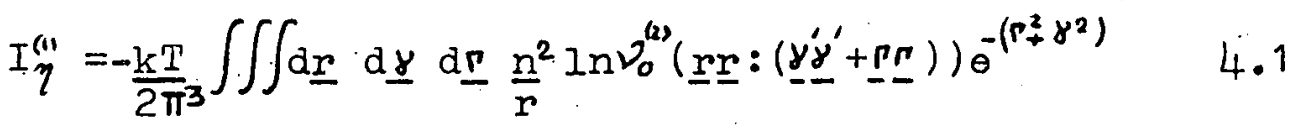

$$
\begin{aligned}
& I=-\frac{k r T}{2 \pi^{3}} \iiint d \underline{r} d \underline{\underline{y}} d \underline{r} n^{2} \ln \nu_{0}^{(2)}\left(\gamma^{2}+r^{2}-\ln \nu_{0}^{(2)}\right) e^{-\left(r^{2}+\gamma^{2}\right)} \quad 4.2
\end{aligned}
$$

The evaluation of $I_{2}^{(2)}$ is relatively simple. Notice that the term $\nu_{0}^{(2)}$ In $p_{0}^{(2)}$ will give an integral proportional to $\dot{B}(T)$, the temperature derivative of the second virial coefficient, which for hard spheres is zero. The term $V_{0}^{(2)} \frac{\partial}{\partial r} \operatorname{In} \nu_{0}^{(2)}$ behaves like Dirac's delta function

$$
\int_{0}^{\infty} d r p_{0}^{(2)} \frac{\partial}{\partial r} \ln p_{0}^{(2)}=1 \quad 4.3
$$

and $\operatorname{dsr}\left(\operatorname{In} \nu_{0}^{(2)} \nu_{0}^{(2)}=\delta(r-\sigma) \quad 4.4\right.$ 
With these results the following expression for $\mathrm{I}_{\eta}^{(2)}$ is obtained

$$
I_{\eta}^{(2)}=-8 \pi n^{2} \sigma^{3} k T
$$

The evaluation of $\mathrm{I}_{\eta}^{(1)}$ is a little more difficult. We first perform the integration over $\square$ to simplify $I_{\eta}^{\prime \prime \prime}$ to

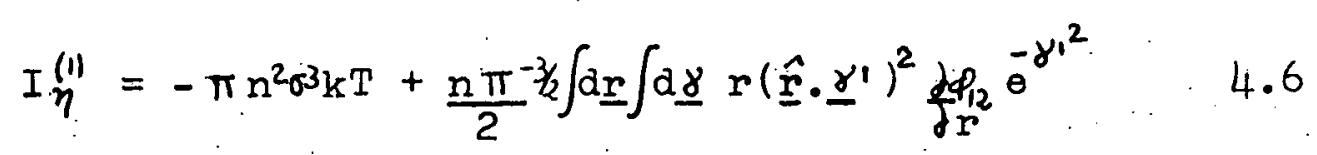

and then change the integration variables to $\left(\underline{\underline{r}}^{\prime}, \underline{\gamma}^{\prime}\right)$. Here $\underline{\gamma}^{\prime}$ is the pre-collision relative velocity and $\underline{r}^{\prime}$ is the relative position vector the colliding molecules would have if $\phi_{12}=0$. Mathematically: $\left(\underline{\gamma}^{\prime}, \underline{r}^{\prime}\right)=\operatorname{Lim}_{\tau \rightarrow \infty} e^{-\tau K_{0}^{(2)}} e^{\tau \underline{v}_{i} \frac{\partial}{\partial \underline{L}}}(\underline{\gamma}, \underline{r})$. The Jacobian of this transformation is unity. The intermolecular potential must now be redefined in terms of the precollision variables. In Appendix A, the following identity is proved

$$
\frac{r}{2 k T} \frac{\partial \phi_{12}}{\partial r}=\underline{\gamma}^{\prime} \cdot \frac{\partial}{\partial \underline{r}^{\prime}}\left(\underline{\gamma}^{\prime} \cdot \underline{r}^{\prime}-\underline{\gamma} \cdot \underline{r}\right)
$$

Due to the delta function behavior of the integrand, $I_{\eta}^{(1)} \neq 0$ only at $\underline{r}=\underline{\sigma}\left(\underline{b}, \underline{\gamma}^{\prime}\right)$. The dependence of $\underline{\sigma}$ on $\underline{b}$ and $\underline{\gamma}^{\prime}$ can be seen in Fig. (4.1)

$$
\underline{\sigma}=\underline{b}-\underline{\hat{\gamma}}^{\prime}\left(\sigma^{2}-b^{2}\right)^{\frac{1}{2}}
$$

In performing the integration over $\underline{r}^{\prime}$, we will choose cylindrical coordinates with the z-axis aligned with the direction 
of $\underline{\gamma}^{\prime} \cdot$ Then $\underline{\gamma}^{\prime} \cdot \frac{\partial}{\partial \underline{r}^{\prime}}=\gamma^{\prime} \frac{\partial}{\partial z}$ and the integration over $z \cdot$ yields

$$
I_{\eta}^{(\prime \prime}=-\pi n^{2} \sigma^{3} k T+8 \pi^{-\frac{1}{2}} n^{2} k T \int_{0}^{J} \int_{0}^{\infty} d b d \gamma b \gamma^{\prime 3}\left(\underline{\tilde{\sigma}} \cdot \underline{\gamma}^{\prime}\right)^{2} e^{-\gamma^{\prime 2}}\left(\left.\underline{\gamma^{\prime}} \cdot \underline{\left.\underline{r^{\prime}}-\underline{\underline{r}} \cdot \underline{\gamma}\right)}\right|_{i=-\infty} ^{z=\infty}\right.
$$

At $z=-\infty, \underline{\gamma}^{\prime} \cdot \underline{r}^{\prime}=\underline{\gamma} \cdot \underline{r}$. At $z=+\infty$, figure $1 . \cdot 1$ shows that

$$
\left.\left(\underline{\gamma^{\prime}} \cdot \underline{\underline{r}}^{\prime}-\underline{\gamma} \cdot \underline{\underline{r}}\right)\right|^{z=+\infty}=-2 \gamma^{\prime}\left(\sigma^{2}-b^{2}\right)^{\frac{3}{2}} \quad 4 \cdot 10
$$

We then substitute Eqs. $(4.8,4.10)$ into $\mathrm{Eq} .(4.9)$ to obtain

$$
I_{\eta}^{\prime \prime}=-l_{1} \cdot \pi n^{2} \sigma^{3} k \mathrm{kT}
$$

For the hard sphere molecular model $\eta$ is obtained from Eq.

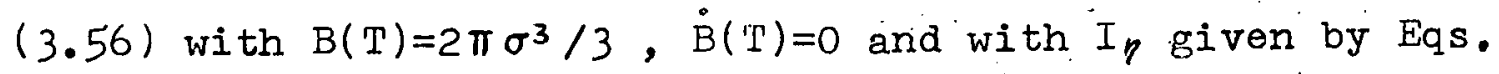
$(4.5,4.11)$ : Thus

$$
\eta=\eta_{0}\left(1+\frac{\left.4 n \pi \sigma^{3}\right)^{2}}{15}=\eta_{0}\left(1+\frac{8 n \pi \sigma^{3}}{15}+\ldots\right) \quad 4.12\right.
$$

In finding $\lambda$ we will not encounter any new problem. Notice that in Eqs (3.61) $\underline{q}_{\varphi}^{(2)}$ is proportional to $\dot{B}(T)$ and that therefore, for this molecular model, is zero. All of the information necessary for the evaluation of $q_{\varphi}^{(\prime \prime)}$ has already been derived in this section. We substitute

$$
\underline{q}_{\varphi}^{(1)}=\left(1 / 2 \pi n^{2} \sigma^{3} \mathrm{kT}\right) a_{1} \nabla \ln \mathrm{T}
$$

into Eq. (3.66) to obtain

$$
\lambda=\lambda_{0}\left(1+\frac{\left.2 n \pi \sigma^{3}\right)^{2}}{5}=\lambda_{0}\left(1+\frac{\ln \pi \sigma^{3}}{5}+\ldots\right) \quad \text { i... } 14\right.
$$

I'he transport coefficients of Eqs. $(4.12,4.14)$ are in agreement with Enskog's to terms linear in the density. 
4.2 The Square Well Potential Model

From the analogous calculation done using the rigid sphere model we have all of the information needed for this new calculation. The choice of the square well potential has been made intentionally since besides allowing bound states in molecules, it avoids mathematical difficulties that, for example, the more realistic Lennard-Jones potential presents. Approximations introduced by the choice of the square well potential are compensated by the relative simplicity of the mathematics involved which allows to solve analytically most of the integrals encountered and to simplify greatiy many others.

This calculation will be useful in establishing the sensitivity of transport virial coefficients to the molecular potential. If $\beta_{\eta}^{*}$ and $\beta_{\lambda}^{*}$ from these calculations should compare well with $\beta_{\eta}^{*}$ and $\beta_{,}^{*}$. from the Lennard-Jones calculation, then we would know that these transport coefficients are not sensitive to the potential and that, therefore, they do not represent a good test for our theory.

In section 3.1 we assumed that $\mathrm{f}_{c}^{(2)}$ is determined by the equation $k_{0}^{(2)} f_{c}^{(2)}=0$ from which we conclude that $f_{c}^{(2)}$ is constant along the two particle trajectory in the relative position-velocity space. As previously discussed, it is assumed that $\mathrm{f}_{c}^{(2)}$ contains no pair correlation in pre-collision states but that such correlation does exist in post-collisional 
states by virtue of the binary collisions. The scattering collisions, therefore, are the randomizing events which drive the system to equilibrium, that is, which force $f_{c}^{(\prime \prime}$, to vanish. Now the bound molecular states do not correspond to scattering collisions and do not have such a randomizing effect. In fact, in a binary collision approximation two bound particles never separate, and, therefore, it seems plausible to assume that $f_{c}^{(2)}=0$ on bounded trajectories. The effects of bounded states are contained entirely into the correlation functions built into $f_{0}^{(2)}$ which appear explicitly in the functions $F_{\eta}(T), F_{\lambda}(I)$ of the linearized Boltmann equation.

In $I_{\eta}$ and $I_{\lambda}$, the integration is over all values of $\underline{r}, \underline{W}_{1}, \underline{W}_{2}$ corresponding to bounded and nonbounded states. Since the effects of bounded states are contained only in a function which does not contribute to the fluxes (i.e. $f_{0}^{(2)}$ ) and since the only contribution of $f^{(2)}$ to $I_{\eta}$ and $I_{\lambda}$ comes from $f_{c}^{(2)}$, we can change the integration variables to $\underline{\underline{x}}^{\prime}, \underline{W}_{1}^{\prime}, \underline{W}_{2}^{\prime}$ and reduce nine fold integrals to two fold integrals with the same technique described in chapter 3.

The square well potential is a spherically symmetric potential defined by

$$
\begin{array}{ll}
\phi_{12}(r)=\infty & 0 \leq r \leq a \\
\phi_{12}(r)=-\epsilon & a \leq r \leq c \\
\phi_{12}(r)=0 & r>c
\end{array}
$$

where a is the diameter of the molecular "hard core" and c 
is the range of the molecular potential. Depending upon the value of the collision parameter $b$, deflection can occur with or without collision between the two molecular hard cores. In the following the values of the impact parameter $b$, which distinguish these two types of collisions are calculated. From the conservation of angular momentum and energy, a relationship between $b$ and $b^{\prime}$ is readily obtained... Fig. (4.2). We

find $b \gamma:=b^{\prime \gamma}$ $4 \cdot 15$

$$
\gamma_{1}^{2}=\gamma_{1}^{2}+\phi \phi_{2} / \mathrm{kT}
$$$$
4.16
$$$$
b=(b ! / \gamma 1)\left(\gamma r^{2}+\epsilon / k T\right)^{\frac{1}{2}}
$$

Define $h$ to be the critical value of $b$ obtained by substituting $b^{\prime}=a$ into Eq. $(4.17)$, then

$$
h\left(\gamma^{\prime}\right)=\left(a / \gamma^{\prime}\right)\left(\gamma^{\prime 2}+\epsilon / k \eta\right)^{\frac{1}{2}} \quad 4.18
$$

'Thus $h\left(\gamma^{\prime}\right)$ is the parameter that distinguishes the two possible types of collisions. That is,

$$
\begin{aligned}
& \text { if } 0 \leq b \leq h\left(\gamma^{\prime}\right) \quad \text { Collision of type one (Fig. (4.2)) } \\
& \text { if } h\left(\gamma^{\prime}\right) \leq b \leq c \quad \text { Collision of type two (Fig. (4.3)) } \\
& \text { if } b>c
\end{aligned}
$$

In collision of type one, the molecules suffer a hard core collision as well as soft potential interaction and $r_{m}$, the distance of closest approach between the two colliding molecules, is equal to a ( see Figs. $(4.2,4.4)$ ). We postpone the 
discussion of the effective potential in Figs. $(4.4,4.5)$ to chapter 5. In collisions of type two, $r_{m}>a$, and only soft. interactions occur. See Figs. $(4.3,4.5)$.

Since $I_{\eta}$ and $I_{\lambda}$ are similar, we will refer our discussion only to the solution of $I_{\eta}(T)$ and then give, at the end of this chapter, expressions for both $B_{\eta^{*}}^{*}\left(T^{*}\right)$ and $B_{\lambda}^{*}\left(T^{*}\right)$. In Eq. (3.53), we change the integration variables to ( $\left.\underline{\underline{y}}^{\prime}, \underline{\gamma}^{\prime}, \underline{\underline{ }}\right)$ and perform the integration over I; using Eq. (4.7) In then becomes

$$
\begin{aligned}
I_{\eta}=n^{2} k T \pi^{-\frac{3}{2}} \iint d \underline{r}^{\prime} d \underline{\gamma^{\prime}} & \left(3\left(\underline{\underline{r}} \cdot \underline{\gamma}^{\prime}\right)+\gamma^{\prime 2}\right) r^{2} e^{-\gamma^{\prime 2}} \\
& \left(\underline{\gamma}^{\prime} \cdot \frac{\partial}{\partial \underline{r}^{\prime}}\left(\underline{\gamma}^{\prime} \cdot \underline{r}^{\prime}-\underline{\gamma} \cdot \underline{r}\right)-\frac{\phi_{12}}{k T}\right) \quad 4.19
\end{aligned}
$$

The integration over the position coordinate can be carried out using cylindrical coordinates, as discussed in section 4.1. In Eq. (4.19) the part of the integrand containing only $\gamma^{\prime 2}$ can be integrated immediately over $z$, in this case we need to find only $\left(\underline{\gamma}^{\prime} \cdot \underline{r}^{\prime}-\underline{y} \cdot \underline{\underline{r}}\right)$ at $z=+\infty$. The other part of the integrand containing ( $\left.\underline{\hat{\hat{r}}} \cdot \underline{\underline{\gamma}^{\prime}}\right)$ cannot be directly integrated over $z$, since $\left(\hat{\hat{\hat{r}}} \cdot \underline{\underline{\gamma}}^{\prime}\right)$ is a function of $z$. We will instead. first find an expression for $\left(\underline{\underline{r}} \underline{\underline{\gamma}}^{\prime}-\underline{r} \cdot \underline{\gamma}\right)$ in terms of $z$ and $\underline{\gamma}^{\prime}$, then perform the derivative with respect to $z$, and finally integrate over $z$.

Vefine $t_{c}$ as the duration of a collision and $z_{0}$ the distance the incoming molecule would have gone in time $t_{c}$ if it had not suffered a collision. Then (see Figs. (4.2, 4.3)) 


$$
z_{0}=t_{c} \gamma^{\prime}
$$

$4 \cdot 20$

and for type one collisions

$$
\begin{array}{ll}
\underline{\gamma}^{\prime} \cdot \underline{\underline{r}}^{\prime}=\underline{\gamma}^{\prime} \cdot \underline{c}+\gamma^{\prime} z & 4.21 \\
\underline{\gamma} \cdot \underline{\underline{r}}=\underline{\gamma} \cdot \underline{c}+\left(z / \gamma^{\prime}\right) \gamma^{\prime 2}, & 4.22
\end{array}
$$

if $0<z<z_{0} / 2$ and

$$
\begin{array}{ll}
\underline{\gamma}^{\prime} \cdot \underline{\underline{r}}^{\prime}=\underline{\gamma}^{\prime} \cdot \underline{c}+\gamma z & 4.23 \\
\underline{\gamma} \cdot \underline{r}=\underline{\gamma} \cdot\left(\underline{a}\left(t_{c} / 2-\left(t_{c}-\bar{t}_{0}\right)\right)\left(\gamma / \gamma^{\prime}\right)\right) & 4.24
\end{array}
$$

for $z_{0} / 2 \leq z \leq z_{0}$

Here, $\tilde{t}_{0}$ is the time elapse since the molecule was at $z=0$. It is more convenient to rewrite Eq. $(4.24)$ in terms of $z$ as follows :

$$
\underline{\gamma} \cdot \underline{r}=\underline{\gamma} \cdot \underline{a}\left(z-z_{0} / 2\right)\left(\gamma^{2} / \gamma^{\prime}\right): \quad: 4.25
$$

In the case $z>z_{0}$ then

$$
\begin{array}{ll}
\underline{\gamma} \cdot \underline{r}=\underline{\gamma} \cdot \underline{r}_{0}+\left(z-z_{0}\right) \gamma^{\prime} & 4.26 \\
\underline{\gamma}^{\prime} \cdot \underline{r}^{\prime}=\underline{\gamma}^{\prime} \cdot \underline{c}+\gamma^{\prime} z_{0}+\left(z-z_{0}\right) \gamma^{\prime} & 4.27
\end{array}
$$

An expression for $z_{0}$ can readily be found from geometrical arguments (see Fig. (4.2)). 'Thus we find that

$$
\begin{array}{ll}
t_{c}=a / \gamma & 4.28 \\
d=2\left(c^{2}-b^{12}\right)^{\frac{3}{2}} & 4.29
\end{array}
$$


Substitution: of Eqs. (4.18, 4.28, 4.29) in Eq. (4.19) yields the desired equation for $z_{0}$

$$
\begin{aligned}
z_{0}=\left(2 \gamma^{\prime} /\left(\gamma^{2}+\epsilon / k T\right)\right)\left(\left(c^{2}\left(\gamma^{\prime 2}+\epsilon / k T\right)-b^{2} \gamma^{\prime 2}\right)^{\frac{1}{2}}-\right. & \\
& \left.\left(a^{2}\left(\gamma^{\prime 2}+\epsilon / k T\right)-b^{2} \gamma^{\prime 2}\right)^{\frac{1}{2}}\right): \quad 4 \cdot 30
\end{aligned}
$$

Using exactly the same geometric and conservation arguments, analogous relationships for $\underline{\gamma} \cdot \underline{\underline{r}}$ and $\underline{\gamma}^{\prime} \cdot \underline{x}^{\prime}$ can be derived in the case of collision of type two. These are, for $z<z o$

$$
\begin{array}{ll}
\underline{\gamma} \cdot \underline{\underline{r}}=\underline{\gamma} \cdot \underline{c}+\left(z \gamma^{2} / \gamma^{\prime}\right) & 4.31 \\
\underline{\gamma}^{\prime} \cdot \underline{r}^{\prime}=\underline{\gamma}^{\prime} \cdot \underline{c}+\gamma_{z} & 4 \cdot 32
\end{array}
$$

and for $z>z_{0}$,

$$
\begin{aligned}
& \underline{\gamma} \cdot \underline{\underline{r}}=\underline{\gamma} \cdot \underline{r}_{0}+\left(z-z_{0}\right) \gamma^{\prime} \\
& \underline{\gamma} \cdot \underline{r}^{\prime}=\underline{\gamma} \cdot \underline{c}+\gamma z_{0}+\left(z-z_{0}\right) \gamma_{1} .
\end{aligned}
$$

The mathematical relationships needed to define ( $\left.\underline{\gamma}^{\prime} \cdot \underline{\underline{r}}^{\prime}-\underline{\gamma} \cdot \underline{r}\right)$ over all possible values of $z$ and $b$ are now available. In tabular form they are:

$$
\begin{array}{llll}
\underline{\gamma} \cdot \underline{c}+\gamma^{\prime} z-\underline{\gamma} \cdot \underline{c}-\left(\gamma^{\prime} / \gamma^{\prime}\right) z, & 0<z<z_{0} / 2,0<b<h\left(\gamma^{\prime}\right) & 4.34 \\
\underline{\gamma} \cdot \underline{c}+\gamma^{\prime} z-\underline{\gamma} \cdot \underline{a}-\left(z-z_{0} / 2\right)\left(\gamma^{2} / \gamma^{\prime}\right), & z_{0} / 2<z<z_{0}, 0<b<h\left(\gamma^{\prime}\right) & 4.35 \\
\underline{\gamma} \cdot \underline{c}+\gamma^{\prime} z-\underline{\gamma} \cdot \underline{\underline{r}}-\left(z-z_{0}\right) \gamma^{\prime}, & z>z_{0}, & 0<b<h\left(\gamma^{\prime}\right) & 4.36 \\
\underline{x} \cdot \underline{c}+\gamma^{\prime} z-\underline{\gamma} \cdot \underline{c}-\left(\gamma^{2} / \gamma^{\prime}\right) z, & 0<z<z_{0}, & h\left(\gamma^{\prime}\right)<b<c & 4.37 \\
\underline{\gamma} \cdot \underline{c}+\gamma^{\prime} z-\underline{\gamma} \cdot \underline{\underline{r}}-\left(z-z_{0}\right) \gamma^{\prime} & z>z_{0} & h\left(\gamma^{\prime}\right)<b<c & 4.38
\end{array}
$$

Let us define a quantity $w_{0}$.by the relation 


$$
w_{0}=\gamma^{\prime} \frac{\partial}{\partial z}\left(\underline{\gamma}^{\prime} \cdot \underline{x}^{\prime}-\underline{\gamma} \cdot \underline{\underline{r}}\right)+\epsilon / \mathrm{kT}
$$

If any of the expressions for ( $\underline{\gamma}^{\prime} \cdot \underline{\underline{r}}^{\prime}-\underline{\gamma} . \underline{\underline{r}}$ ) given by E'qs. (4.34, 4.38) are substituted into $\mathrm{Eq}$. (4.39) the result that wo as defined above behaves like a Dirac delta function is obtained. This result is not surprising since wo represents an expression for $\frac{\partial}{\partial r}$, where $\phi$, the square well potential, is a step function. These last remarks suggest another equivalent way in which $w_{0}$ can be written; namely

$$
\begin{array}{ll}
w_{0}=a_{1} \delta(z)+a_{2} \delta\left(z-z_{0}\right), \text { if } b>h\left(\gamma^{\prime}\right), & 4.40 \\
w_{0}=c_{1} \delta(z)+c_{2} \delta\left(z-z_{0} / 2\right)+c_{3}\left(z-z_{0}\right) ; \text { if } b\left\langle h\left(\gamma^{\prime}\right)\right. & 4.41
\end{array}
$$

The mathematical method used to find the constant $\delta$-function strength are given in Appendix $B$. We find that

$$
\begin{aligned}
& a_{i}=a_{2}=c_{1}=c_{3}=\gamma^{\prime}\left(c^{2}\left(\gamma^{\prime}+\epsilon / k T\right)-\gamma^{\prime 2} b^{2}\right)^{\frac{1}{2}}-\gamma^{\prime 2}\left(c^{2}-b^{2}\right)^{\frac{1}{2}} 4.42 \\
& c_{2}=-2 \gamma^{\prime}\left(a^{2}\left(\gamma^{\prime 2}+\epsilon / k T\right)-\gamma^{\prime 2} b^{2}\right)^{\frac{1}{2}}
\end{aligned}
$$

These last two equations contain the final pieces of information needed to redefine the integrand of $I_{\eta}$ explicitly in terms of the variables of integration $b, z$, and $\gamma^{\prime}$.

\subsection{Transport Coefficients for the Square Well Potential}

For convenience, we rewrite $I_{\eta}$ as

$$
I_{\eta}=\pi^{-\frac{3}{2}} n^{2} k T\left(3 I_{\eta}^{(1)}-I_{\eta}^{(2)}\right)-\gamma^{\prime 2}
$$

where $I_{\eta}^{(\prime)}=\iint d \underline{\gamma}^{\prime} d \underline{\underline{p}}^{\prime} \cdot w_{0}\left(\underline{\hat{\underline{r}}} \cdot \underline{\gamma}^{\prime}\right) \mathrm{e}^{-\gamma^{\prime}}$

$$
I_{\eta}=\iint d \underline{\gamma}^{\prime} d \underline{r}^{\prime} w_{0} \gamma^{2} e^{-\gamma^{\prime 2}}
$$


'The integration over $\underline{r}^{\prime}$ in $\mathrm{J}_{\eta}^{(2)}$ is easily carried out in cylindrical coordinates. With the help of Eqs. (4.34., 4.35, 4.36, $4.37,4.38), I_{\eta}^{(2)}$ becomes

$$
I_{\eta}^{(2)}=-c \pi^{2}\left(\frac{8 \epsilon}{3 k T}+5 \pi^{3 / 2}\right)+\frac{16}{3} \pi^{2}\left(c^{2}-a^{2}\right) \int_{0}^{\infty} d \gamma^{\prime} \gamma^{\prime 3}\left(\gamma^{\prime 2}+\frac{\epsilon}{k T}\right) e^{-\gamma^{\prime 2}} 4.44
$$

The evaluation of $I_{\eta}^{(1)}$ is complicated by the factor $\left(\underline{\underline{r}} \cdot \underline{\gamma}^{\prime}\right)$ in the integrand. Besides being a function of $z$, this quantity assumes different forms for different types of collisions. We decompose the integration over $b$ into two parts $(0<b<h$ and $h(b<c)$ containing values of $b$ which correspond to the two types of collisions. Using Eqs. $(4.40,4.41,4.42,4.43)$ we then write

$$
\begin{aligned}
& I_{\eta}^{(1)}=2 \pi \quad\left(\int_{d} \underline{\gamma}^{\prime} \int_{0}^{c} d b b c_{1}\left(\underline{\hat{c}} \cdot \underline{\gamma}^{\prime}\right)^{2} e^{-\gamma^{\prime 2}}+\int d \underline{\gamma^{\prime}} \cdot \int_{0}^{h\left(\gamma^{\prime}\right)} d b b c_{2}\left(\underline{\hat{a}} \cdot \underline{\gamma}^{\prime}\right)^{2} e^{-\gamma^{\prime}}\right. \\
& \left.\int d \underline{\gamma}^{\prime} \int_{0}^{h\left(\gamma^{\prime}\right)} d b b c_{3}\left(\underline{\hat{r}_{0}} \cdot \underline{\gamma}^{\prime}\right)^{2} e^{-\gamma^{\prime 2}}+\int d \underline{\gamma}_{h\left(\gamma^{\prime}\right)}^{c} d b e^{-\gamma^{\prime 2}} b a_{2}\left(\hat{\underline{r}}_{0} \cdot \underline{\gamma}^{\prime}\right)^{2}\right)
\end{aligned}
$$

From Figs. $(4.3,4.3)$ we have

$$
\underline{\hat{c}} \cdot \underline{\gamma}^{\prime}=\gamma^{\prime} \cos \left(\frac{\pi}{2}+\cos ^{-1}\left(\frac{b}{c}\right)\right)=-\left(\gamma^{\prime} / c\right)\left(c^{2}-b^{2}\right)^{\frac{1}{2}}
$$

Using the law of cosines and the principle of energy and momentum conservation, other important relations can easily be derived. In the case of collisions of type one, we find that

$$
\begin{aligned}
& \underline{a} \cdot \underline{\gamma}^{\prime}+\gamma^{\prime} \cos \alpha \\
& \cos \alpha=(b / c)\left(1-\cos ^{2} \theta\right)-\left(1-b^{2} / c^{2}\right)^{\frac{1}{2}} \cos \theta \\
& \cos \theta=(1 / a c)\left(b^{\prime 2}+\left(c^{2}-b^{\prime 2}\right)^{\frac{1}{2}}-\left(a^{2}-b^{\prime 2}\right)^{\frac{1}{2}}\right) \\
& b^{\prime}=b \gamma^{\prime}\left(\gamma^{\prime 2}+\epsilon / k T\right)
\end{aligned}
$$




$$
\underline{r}_{0} \cdot \underline{\gamma}^{\prime}=\gamma^{\prime} \cos B
$$

and

$$
\begin{aligned}
\cos \beta & =\cos \left((\pi-\theta)-\sin ^{-1}(b / c)\right) \\
& =(2 b c)\left(1-\cos ^{2} \theta\right)^{\frac{1}{2}} \cos \theta-\left(2 \cos ^{2} \theta-1\right)\left(1-b^{2} / c^{2}\right)^{\frac{1}{2}}
\end{aligned}
$$

In the case of collisions of type two, we have

$$
\underline{r}_{0} \circ \underline{\gamma}^{\prime}=\gamma^{\prime} \cdot\left(\frac{2 b b^{\prime}}{c}\left(1-b^{\prime 2} / c^{2}\right)-\frac{1}{c}\left(2 b^{\prime 2}-c^{2}\right)\left(1-b^{2} / c^{2}\right)^{\frac{1}{2}}\right)
$$

An enalytical solution to $I_{\eta}^{(1)}$ is impossible, and it is necessary to evaluate $I_{\eta}^{(1)}$ (and $I_{\eta}^{(2)}$ ) numerically. These calculations will be discussed in chapter 6 .

For the square well potential it is easily shown that the second virial coefficient and its temperature derivatives are of the form

$$
\begin{array}{ll}
B(T)=\left(\left(2 / 3^{\prime} a^{3} \pi\right)\left(1+\left(c^{3} / a^{3}-1\right)\left(1-e^{\epsilon / k T}\right)\right)\right. & 4.46 \\
T \dot{B}(T)=(2 \pi / 3)(\epsilon / k T)\left(c^{3}-a^{3}\right) e^{\epsilon / k T} & 4.47 \\
T^{2} \ddot{B}(T)=-(2 \pi / 3)\left(c^{3}-a^{3}\right)(2+\epsilon / k T)(\epsilon / k T) e^{\epsilon / k T} & 4.48
\end{array}
$$

When Eqs. (4.44, 4.45, 4.46, 4.47, 4.48) are substituted into Eq. (3.49) we obtain the final form of $\beta_{7}^{*}\left(T^{*}\right)$ to he used in the numerical calculation. As mentioned previously the evaluation of $I_{\lambda}$ is similar to the evaluation of $I_{\eta}$. In this case we find that

$$
\begin{aligned}
I_{\lambda}=16 k T \pi^{2}\left(I_{\eta}^{(1)}+\right. & \left.\frac{\left(d^{3}-a^{3}\right)}{6}\right) \int_{0}^{\infty} d \gamma^{\prime} \gamma^{\prime}\left(5-\gamma^{\prime 2}\right)\left(\gamma^{\prime 2}+\epsilon / k T\right)^{\frac{3 k}{2}} e^{-\gamma^{\prime 2}}+ \\
& \left.\quad 5 c^{3} \int_{0}^{\infty} d \gamma^{\prime} \frac{\left(2 \gamma^{\prime 2}\right.}{3}-1\right) \frac{(\epsilon / k T)^{\frac{5}{2}} \gamma^{\prime 2} e^{-\gamma^{\prime 2}}}{\left(\gamma^{\prime 2}+\epsilon / k T\right)}-\frac{c^{3}}{32}\left(5 \pi^{\frac{1}{2}}+\frac{32}{3}\left(\frac{\epsilon}{k T}\right)^{3 / 3}\right)
\end{aligned}
$$




$$
\left.-\frac{5}{4}\left(c^{3}-a^{3}\right) \quad d \gamma^{\prime}(\epsilon / k T)\left(\gamma^{\prime 2}+\epsilon / k T\right)^{\frac{1}{2}}\left(\frac{2}{3} \gamma^{\prime 2}-1\right) \gamma^{\prime} e^{-\gamma^{2}}\right) 4 \cdot 49
$$

'The double integrals appearing in $I_{\lambda}$ can be written in terms of $I_{\eta}^{(1)}$. Hence computation of $I_{\lambda}$ involves only three new one dimensional integrals. 


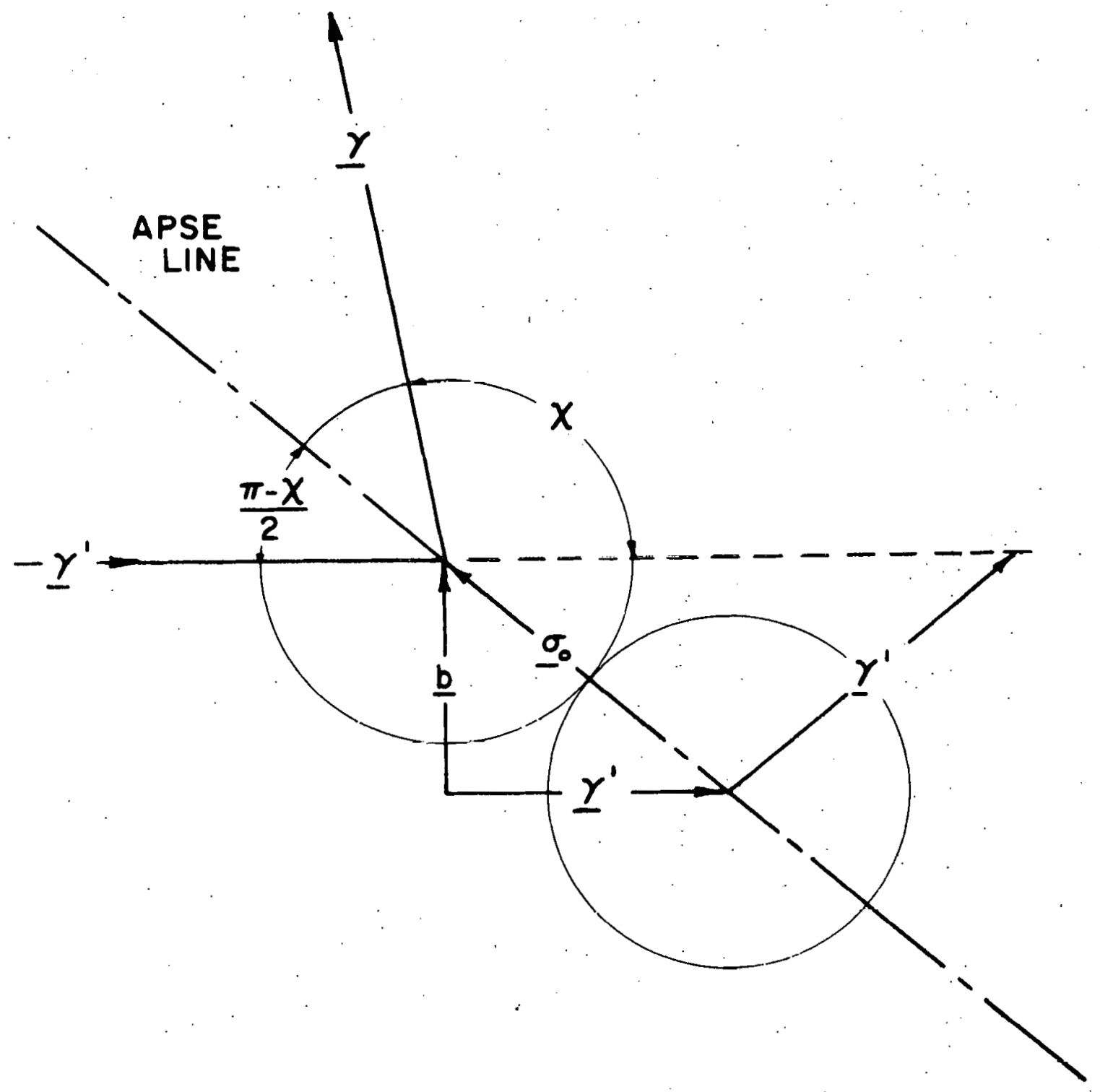

Figure 4.1 Collision of two hard spheres 


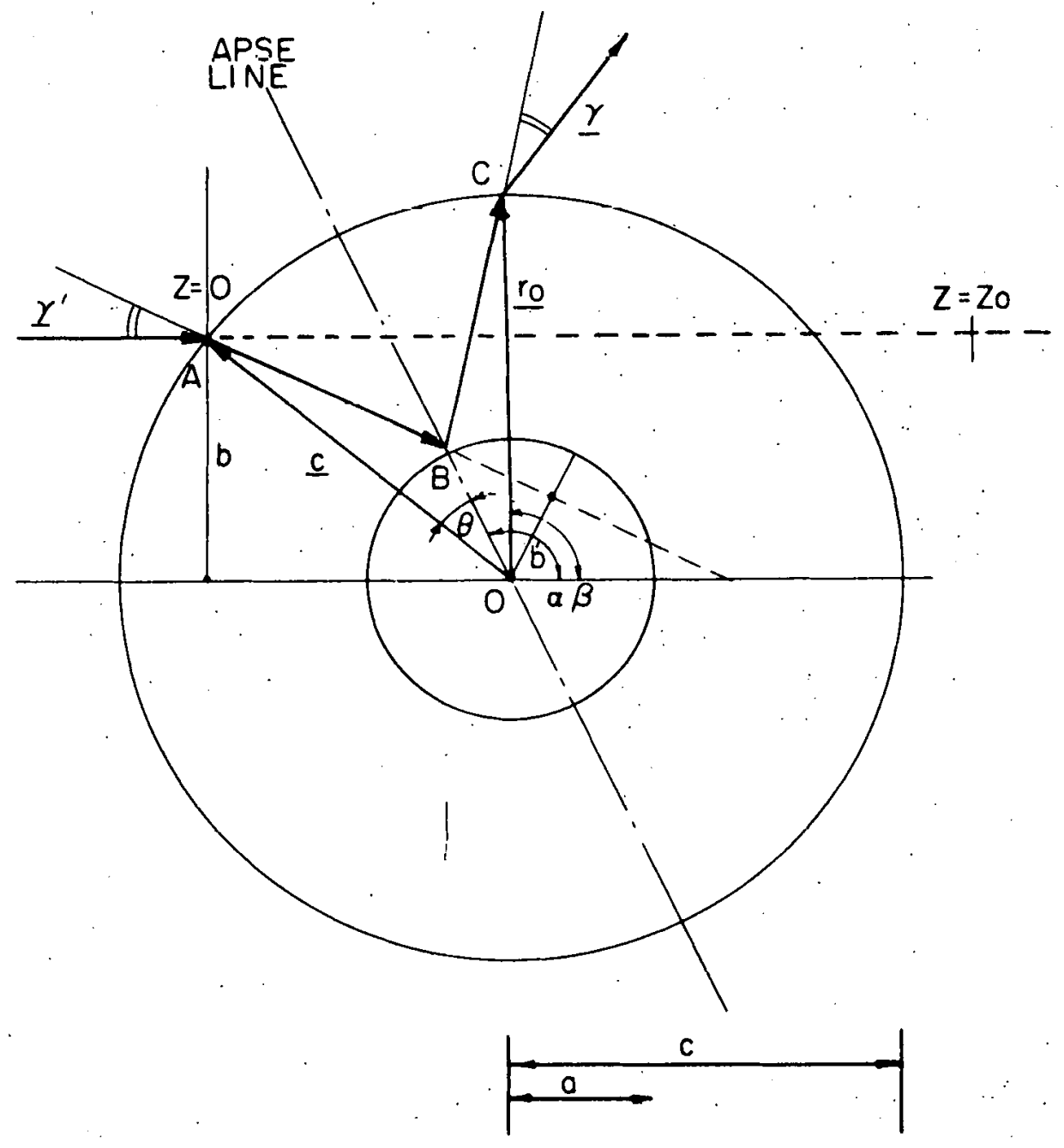

Figure 1..2 Collision of type one. ABC is the trajectory of the center of mass of particle 2 relative to particle 1 whose mass center is at 0 . 


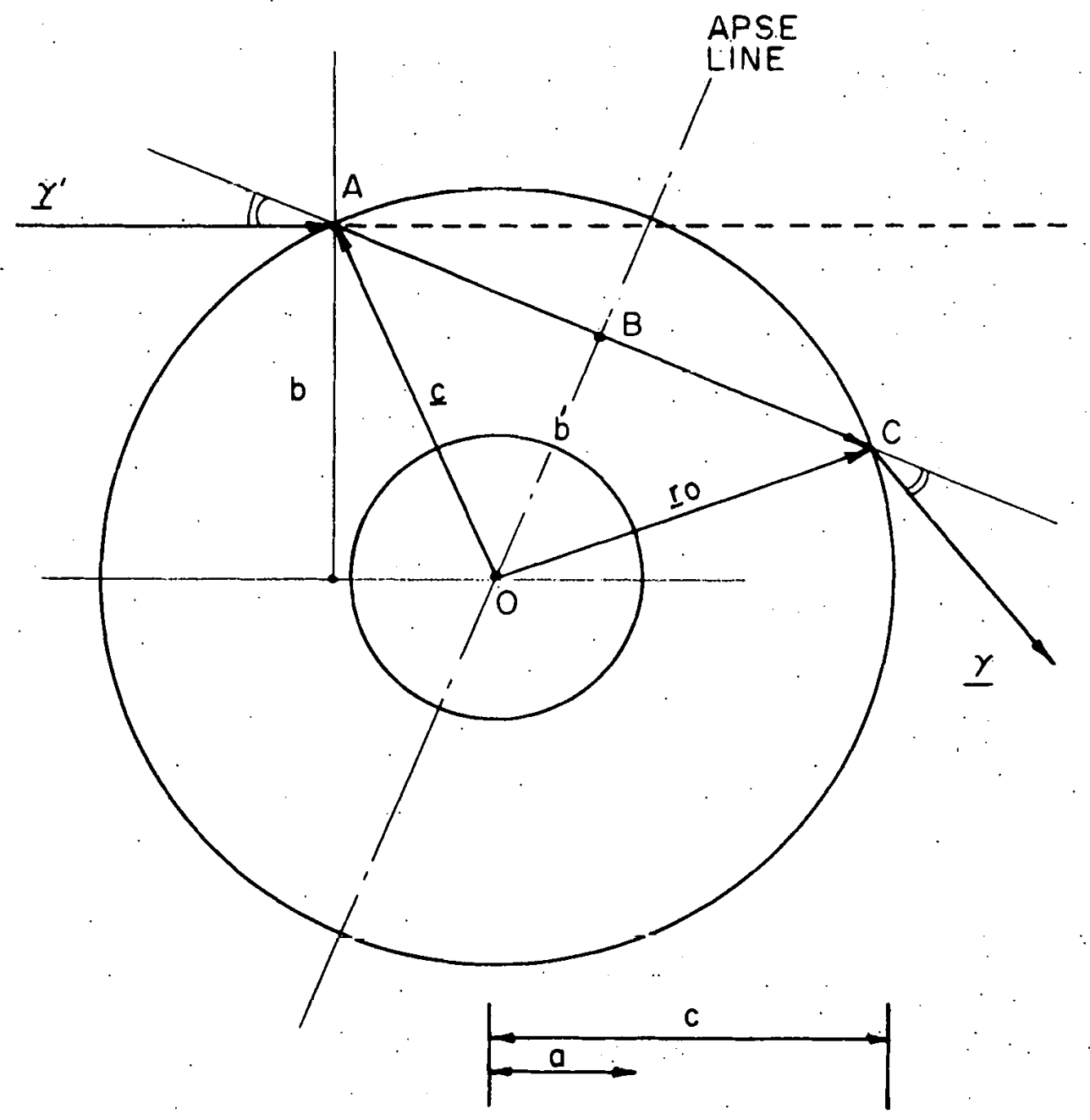

Figure 4.3 Collision of type two. ABC is the trajectory of the center of mass of particle 2 relative to particle 1 whose mass center is at 0 . 


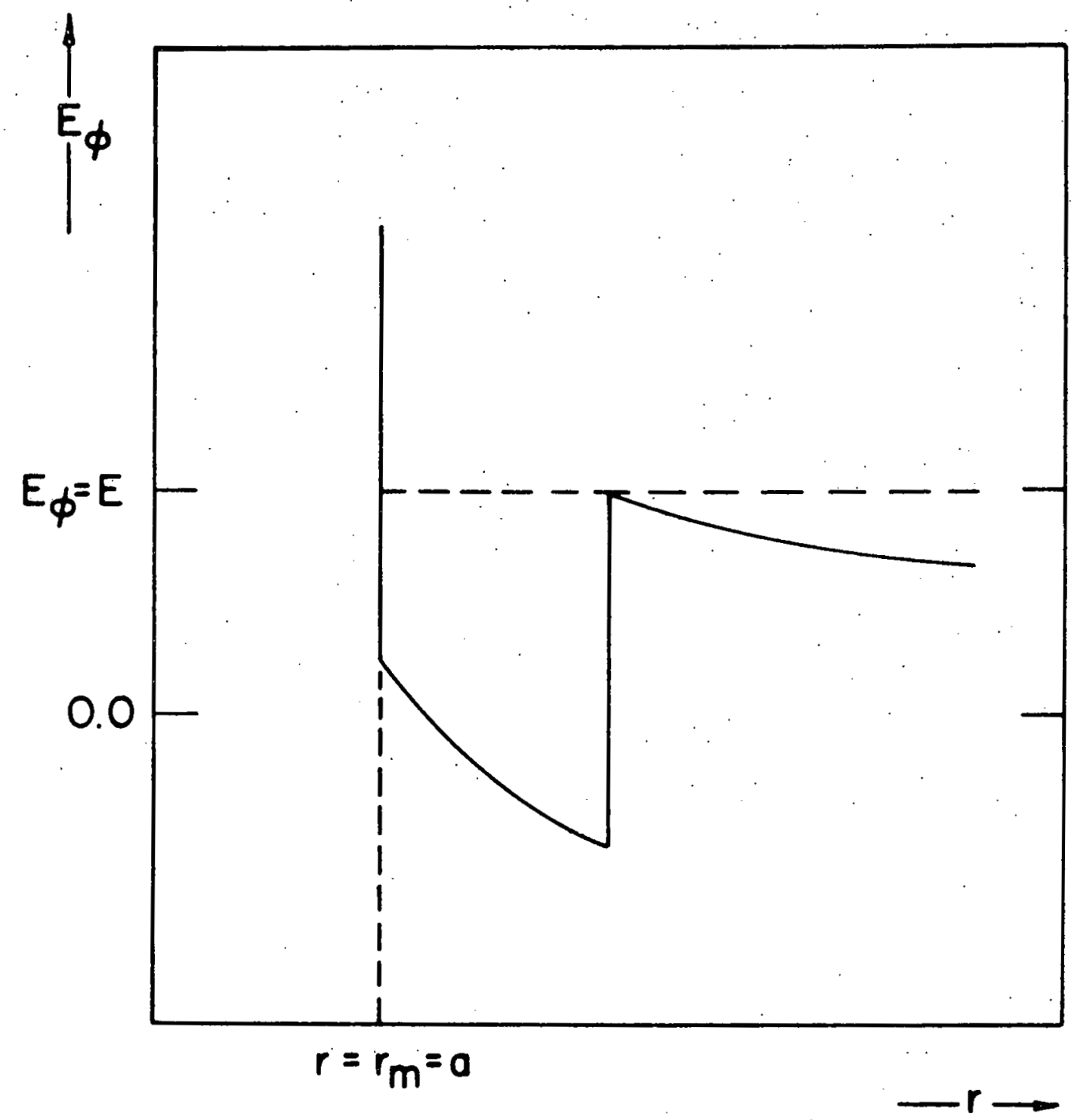

Figure 4.4 The effective potential curve $E_{\phi}$ for the square we $\perp \perp$ potential when $\dot{0}<b<h\left(y^{i}\right)$ and $r_{m}=a$. 


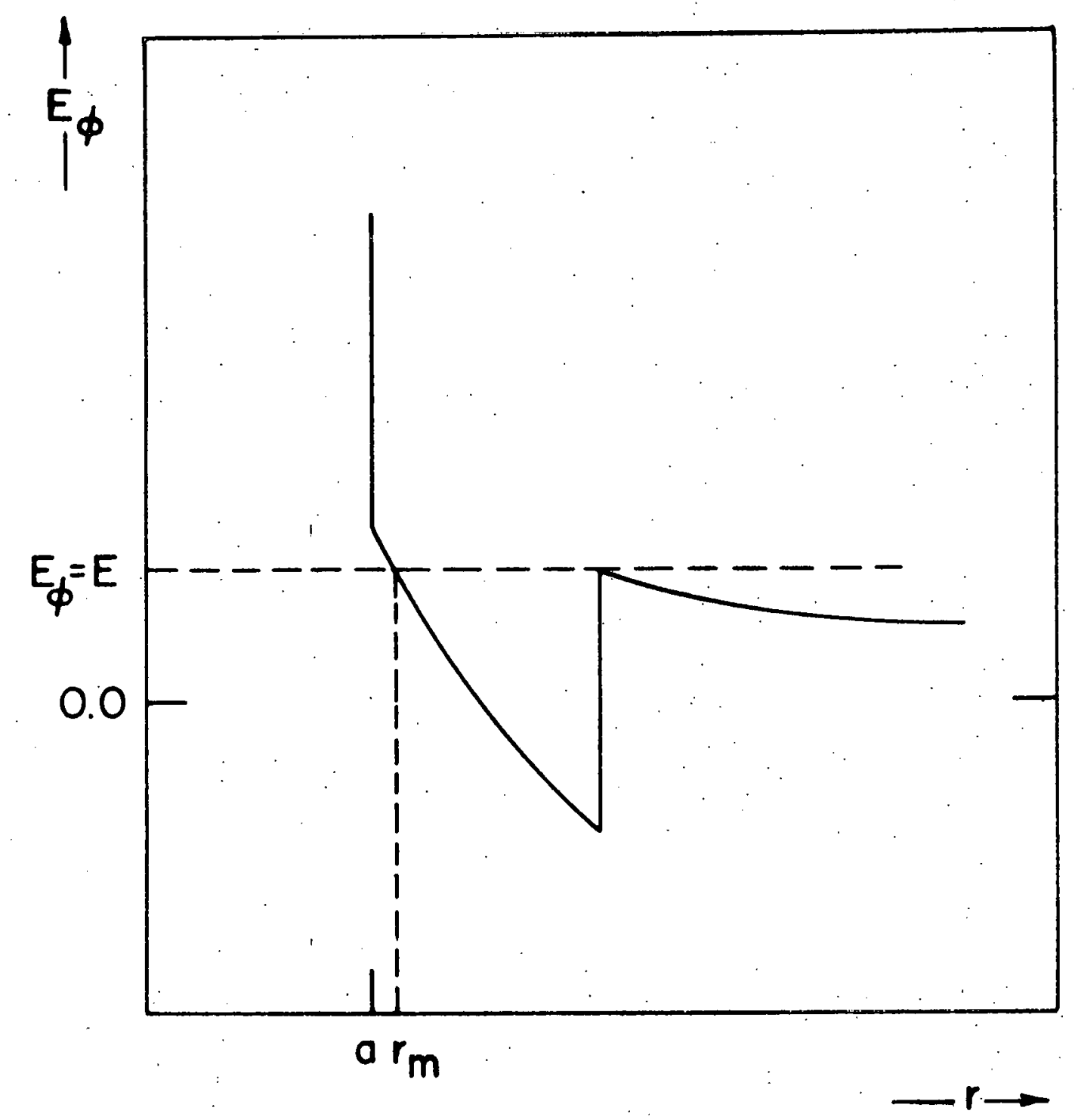

Pigure 4.5 The effective potential curve Eq for the square 
5. TRANSPORT COEFFICIENT'S FOR THE LENNARD-JONES MODEL

5.1 The Description of the Dynamics of Collision (35)

In order to evaluate the fluxes and therefore transport coefficients, it is necessary to solve the dynamical problem of the collision of two molecules of equal mass interacting through the central force potential $\varphi$. As was shown in the previous chapter, this is a fairly easy problem for the square well potential because collision trajectories can be decomposed into linear segments. For a continously varying potential, such as the Lennard-Jones potential, the problem is more complicated.

The Lennard-Jones potential is defined as follows:

$$
\Phi(r)=\epsilon F(r / \sigma), \quad F(r / \sigma)=4\left((\sigma / r)^{12}-(\sigma / r)^{6}\right) \quad 5.1
$$

where $\epsilon$, as before, is the "depth of the well"; that is the value of $\varphi(r)$ at the minimum of the $\varphi(r)$ vs. $r$ curve. $\sigma$ is the value of $r$ when $\varphi=0$.

Due to the complexity of the collision dynamics it is found to be convenient to introduce a new set of integration variables for the evaluation of the integrals $I_{\eta}$ and $I_{\lambda}$. In this section we describe the various possible types of binary collisions which can occur for molecules which interact through the Lennard-Jones potential. The parametrization of these collisions is the basis for the choice of the new integration variables. 
As is well known, the collision between two identical particles interacting via a central force field takes place in a plane of fixed orientation which translates with the constant velocity of the center of mass. The angular momentum $\underline{L}$, and the energy $E$ of the motion relative to the center of mass, are conserved; $\underline{L}$ is a vector normal to the plane of collision. Ihese two constants of the motion can be written in the form

$$
\begin{array}{ll}
\underline{L}=\frac{m}{2} \underline{r} \times \underline{V}_{12}, L=\frac{m}{2} b v_{12}^{\prime} & 5.2 \\
E=\frac{m}{4} \dot{r}^{2}+\left(\frac{L^{2}}{m r^{2}}+\varphi(r)\right) & 5.3
\end{array}
$$

where $\dot{r}$ is the time rate of change of the particle separation $r ; \frac{m \dot{r}^{2}}{4}$ is the radial kinetic energy $E_{r}$. The rotational kinetic energy $\frac{\mathrm{L}^{2}}{\mathrm{mr}^{2}}$ in Eq. (5.3) has been written here in terms of $L$ and $r$, and since the angular momentum for a given collision is conserved the rotational kinetic energy on a given trajectory varies only with $r$. Therefore, $E$ can be thought of as the Hamiltonian of a hypothetical one dimensional problem for the scattering of a particle of mass $\frac{m}{2}$ with position $r$, and velocity $\dot{r}$, from a scatterer located at the origin which interacts with the particle via the effective potential, $E_{\varphi}=\frac{L^{2}}{\mathrm{mr}^{2}}+\phi(r)(F i g \cdot(5.1))$. It is convenient to write Eq. $(5.3)$ in the following reduced form

$$
\gamma^{\prime 2}=\frac{m \dot{r}^{2}}{4 k T}+\gamma^{\prime 2}(b / r)^{2}+\phi(r) / k T+5.4
$$

A plot of $E_{\varphi}$ vs. $r$ gives a curve parameterized by $E b^{2}=\frac{L^{2}}{m}$. 
Different one dimensional interaction potentials are possible, for different values of this parameter. Fig. (5.2) shows a collection of such curves. Note the existence of a critical value $\left(E \dot{b}^{2}\right)_{c}$ at which an inflection point occurs. For all curves with $\left(E b^{2}\right)>\left(E b^{2}\right)_{c}, E_{\phi}(r)$ is a monotonically decreasing function of $r$ and behaves as if it were a repulsive potential. For $\left(E b^{2}\right)<\left(E b^{2}\right)_{c}$, curves have both a maximum which goes to zero as $\left(\mathrm{Eb}^{2}\right) \rightarrow 0$ (head-on collision), and a minimum which goes to $-\epsilon$ as $\left(E b^{2}\right) \rightarrow 0$. Fig. (5.2) has been reproduced from as article by D. E. Stogryn and J. 0. Hirshfelder (36). For values of $\left(E b^{2}\right)<\left(E b^{2}\right)_{c}$ trajectories exist for which molecules can orbit around the scatterer. In Fig. (5.3) the shaded area represents values of $E_{\varphi}$ and $r$ corresponding to these orbital motions; if the total energy $E$ should be slightly greater than the maximum of the Es vs. $r$ curve, the colliding molecule after orbiting for some time, will go over the "hump", reach a distance of closest approach from the scatterer $r_{1}$, with $r_{i}=r_{m}$ ( $r_{m}$ is, as before, the largest turning point, and in this case $r_{i}$ is the only turning point), and then turn away. If E should be slightly smaller than the value of $E_{\phi}$ at the "hump", the molecule after orbiting the scatterer with an orbit of radius $r_{0}$ will leave the potential field, in this case $r_{m}=r_{0}, r_{0}>r_{t}$. Therefore, for each trajectory with $\left(E^{2}\right)<\left(E b^{2}\right)_{c}$, there exists an energy $E$ equal to Ex at the "hump" for which two possible values of $r_{m}$ can exist. As we later discuss, the collection of these points 
define the curve BCD in Fig. (5.4), which is a plot of $\frac{E}{E}$ vs. z, $z=x_{m} / \sigma$. A point in the shaded area of Fig. (5.4) gives either a turning point and energy for which there is no trajectory (as in the area under $A B$. for which the kinetic energy would be negative), or if the trajectory exists the turning points are either inner or outer turning points of a bound state, as in the area under $B C D$. Any point in the nonshaded area of Fig. (5.4) corresponds to the energy and turning point of a nonbounded trajectory.

Line $A B$ is a portion of the curve $E_{\varphi}$ vs. r for the case of a head-on collision (i.e., $E_{\phi}=\Phi$ ). That is

$$
\mathrm{v}^{*^{2}}=\mathrm{F}(\mathrm{z}), \quad \mathrm{z}<\mathrm{z}_{i}, \quad \boldsymbol{*}^{2}=E / \epsilon \quad 5.5
$$

Curve $C D$ is the locus of the outer turning points $r_{0}$ for trajectories with $\left(E b^{2}\right)<\left(E b^{2}\right)_{c}$ and with $E$ equal to $E_{\phi}$ at the "hump". For every turning point on $C D$, the radial component of the kinetic energy vanishes and the effective potential is a maximum. That is

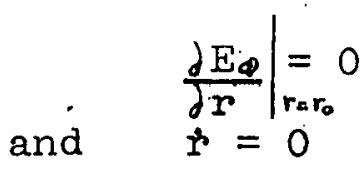

These two equations can be used to determine the impact parameter of the trajectories corresponding to the line CD. Thus we find that

$$
b(E, z)=\left(F^{\prime}\left(z_{0}\right) z_{o}^{3} / 2 E\right)^{\frac{1}{2}}, F^{\prime}=\frac{\partial F}{\partial z} \ldots 5.6
$$


Ihis result then can be substituted into Fq. (5.4) to find the equation for the curve $C D$ which is

$$
\mathrm{v}^{*^{2}}=F\left(z_{0}\right)+\frac{z_{0}}{2} F^{i}\left(z_{0}\right)
$$

A point $\mathrm{E} / \mathrm{e}, \mathrm{z}_{0}$ on the curve $\mathrm{BC}$ is determined by the relation

$$
E_{\Phi}\left(z_{i}\right)=E_{\Phi}\left(z_{0}\right)=E \quad 5.8
$$

where $E / \epsilon, z_{0}$ is a point on CD. Eq. (5.8) can be written in the form

$$
P_{z}=z_{0}^{12}+\frac{12 z_{0}^{8}}{z_{i} F\left(z_{i}\right)}-\frac{8 z_{0}^{6}}{F\left(z_{i}\right)}-\frac{24 z_{0}^{2}}{z_{i} F\left(z_{i}\right)}+\frac{20}{F\left(z_{i}\right)}=0 \quad 5.9
$$

where $P_{z}$ is a 12th degree polynomial in $z_{0}$. The behavior of $P_{z}$ for three values of $z=z_{i}$ is shown in Fig. (5.5); determination of the roots of $\mathrm{P}_{Z}$ is discussed elsewhere (37). A graph of $z_{0}$ vs. $z_{i}$ is given in Fig. (5.6). Since $z_{i}$ and $z_{0}$ are two points on the same trajectory, they must have the same impact parameter. If we substitute $z_{0}$ obtained from the solution of $\mathrm{P}_{\mathrm{z}}$ into $\mathrm{Eq}$. (5.6) to determine $\mathrm{b}$, then we can substitute this value of $b$ into E'q. (5.3) to obtain the result

$$
\mathrm{V}^{2}=\mathrm{F}^{\prime}\left(\mathrm{z}_{i}\right)+\frac{\mathrm{z}_{0}^{3}}{2 \mathrm{z}_{i}^{2}} \mathrm{~F}^{\prime}\left(\mathrm{z}_{0}\right)
$$

This completes the determination of the curve BCD. The curve of $\frac{E}{E}$ vs. $z$, in Fig. (5.4), has a maximum at which $z_{1}=z_{0}=z^{*}$. 'This maximum can be determined from the relation 


$$
\left.\frac{\partial}{\partial z_{0}}\left(F\left(z_{0}\right)+\frac{z_{0}}{2} F^{\prime}\left(z_{0}\right)\right)\right|_{z_{0}=z^{*}}=0
$$

At the maximum we find that

$$
z^{*}=(5)^{1 / 6}, \quad v_{c}^{*^{2}}=4 . / 5, \quad\left(b v^{*}\right)_{c}^{2}=(36 / 25) 5^{1 / 3} \quad 5.11
$$

Eqs. $(5.5,5.7,5.10,5.11)$ contain the information necessary to numerically calculate $B_{\eta}^{*}\left(T^{*}\right)$ and $B_{\eta}^{*}\left(T^{*}\right)$.

5.2 Reduction of the Virial Coefficients to Computational Forms

We write I $\eta$ as given in Eq. (3.53) in terms of spherical coordinates as follows

$$
I_{\eta}=\frac{4}{15 k T} \pi^{\frac{1}{2}} \int_{r_{m}}^{\infty} d r r^{3} \int_{0}^{\infty} d \gamma \gamma^{2} \int_{0}^{\pi} d \theta \sin \theta \gamma^{2^{2}} \frac{\partial \phi}{\partial r}\left(3\left(\underline{\hat{r}} \cdot \underline{\gamma}^{\prime}\right)^{2}-1\right) \theta^{-\gamma^{2}} 5.12
$$

where $\theta$ is the angle between the vectors $\underline{r}$ and $\underline{-\gamma}$. The integration over $\theta$ can be simplified by using the fact that the trajectory is symmetric about the apse iine (38). We then decompose $I_{\eta}$ into the sum of two integrals, one evaluated on the incoming trajectory, which is that part of the trajectory for which

$$
\underline{\hat{r}} \cdot \underline{\hat{\gamma}}^{\prime}=\cos \alpha, 0<\theta<\pi / 2
$$

and the other on the outgoing trajectory, that is where

$$
\underline{\hat{r}} \cdot \underline{\hat{\gamma}}^{\prime}=-\cos (\alpha-2 d m) \quad, \quad \pi / 2<\theta<\pi \quad 5.14
$$

In E'q. $(5.14) \mathrm{dm}$ is the largest value of $\alpha$. This occurs when $r=r_{m}$, Fig. (5.1). Using Eqs. $(5.13,5.14)$ we can write $I_{\eta}$ in 
the form

$$
I_{\eta}=\ldots \int_{0}^{\pi / 2} d \theta \sin \theta\left(3 \cos ^{2} \alpha-1\right)+\ldots \int_{\pi / 2}^{\pi} d \theta \sin \theta\left(3 \cos ^{2}(\alpha-2 \alpha m)-1\right)
$$

5.15 If we make the change of variable $\psi=\pi-\theta$ in the second $r . h . s$. integral, then the two integrals in Eq. (5.15) can be evaluated on the same interval, i.e., $0 \leqslant \psi \leqslant \pi / 2$. Introducing the following reduced vartables

$$
\begin{array}{ll}
\mathrm{x}=\mathrm{r} / \sigma & \mathrm{v}^{*}=\mathrm{T}^{\frac{1}{2} \gamma} \gamma=\mathrm{v} \\
\mathrm{T}^{*}=\mathrm{k}^{\mathrm{l}} \mathrm{l} / \epsilon & \mathrm{F}(\mathrm{x})=\Phi(\mathrm{x}) / \epsilon
\end{array}
$$

Eq. $(5.15)$ can be transformed to

$$
\begin{gathered}
I_{\eta}=\frac{4 \sigma^{3} \pi^{1 / 2}}{15 T^{* 7 / 2}} \int_{0}^{\pi / 2} \int_{0}^{\infty} \int_{2}^{\infty} d \psi d v d x v^{2} v^{2} x F(x) \sin \psi\left(3 \cos ^{2} \alpha+\right. \\
\left.3 \cos ^{2}(\alpha-2 \alpha m)-2\right) e^{-v^{\prime 2 / T^{*}}}
\end{gathered}
$$

The integration variable $x$ fixes a point on a trajectory characterized by the parameters $(v, \psi)$. An integration over all values of $v, x$ and $\psi$, as given by the limit of integration in Eq. (5.16) would include also bound states (b.s.). Since b.s. do not contribute directly to the fluxes we change the integration variables to $\left(v^{\prime}, z, x\right)$ and then use Fig. (5.4) to restrict the limit of integrations of $\mathrm{v}^{\prime}$ and $\mathrm{z}$ to nonbounded states only. The Jacobian of the transformation

$$
\begin{aligned}
& (v, \psi, x) \rightarrow\left(v^{\prime}, z, x\right) \text { is }(39) \\
& \qquad|J|=\frac{\partial\left(v^{\prime}, z, x\right)}{\partial(v, \psi, x)}=\frac{v H(v, z)}{G(x, v, z)\left(v^{2}-F(x)\right)^{1 / 2}\left(v^{2}-F(z)\right)^{1 / 2}}
\end{aligned}
$$


where: $\mathrm{H}(\nabla, z)=v^{2}-F(z)-z F^{\prime}(z) / 2$

$$
\begin{aligned}
& G(v, z, x)=\left(x^{2}\left(v^{2}-F(x)\right)-z^{2}\left(v^{2}-F(z)\right)\right)^{\frac{1}{2}} \\
& v^{2}=v^{2}-F(x) \\
& \left(v^{2}-F(z)\right)^{\frac{1}{2}}=(x / z)\left(v^{2}-F(x)\right)^{\frac{1}{2}} \sin \psi
\end{aligned}
$$

With the new integration variables I $\eta$ becomes

$$
I_{\eta}=a\left(T^{*}\right) \int_{0}^{\infty} d v \int_{0}^{\infty} d z \int_{z}^{\infty} d x x^{2} v^{3} F^{\prime}(x) \frac{A(x, z, v) H(z, v)}{G(x, z, v)} e^{-v^{2} / T^{*}} 5.17
$$

where: $a\left(T^{*}\right)=\frac{4 \pi^{*}}{15} T^{*-1 / 2}$

$$
\begin{aligned}
& A(z, v, x)=3 \cos ^{2} \alpha+3 \cos ^{2}(\alpha-2 \alpha m)-2 \\
& \alpha=z\left(1-F^{\prime}(z) / v^{2}\right)^{\frac{1}{2}} \int_{x}^{\infty} \frac{d y}{y}\left(1-\frac{F(y)}{v^{2}}-\frac{z^{2}}{y^{2}}\left(1-\frac{F(z)}{v^{2}}\right)^{\frac{3}{2}} 5.18\right.
\end{aligned}
$$

and $\alpha_{m}$ is obtained from Eq. (5.18) with $z$ replacing $x$ in the lower limit of integration.

Similarly, for the thermal conductivity coefficient we find that

$$
I_{\lambda}=2 a\left(T^{*}\right) T^{*} \int_{0}^{\infty} d v \int_{0}^{\infty} d z \int_{z}^{\infty} d x \operatorname{vzx} \frac{B(x, z, v) H(z, v)}{G(x, z, v)} e^{-v^{2} / T *} 5.19
$$

where $B(x, z, v)=\left(x F^{\prime}(x)\left(\frac{5}{2}-\frac{v^{2}}{T^{*}}\left(1+\cos ^{2} \alpha+\cos ^{2}(\alpha-2 \alpha m)\right)\right)+\right.$

$$
\left.5 F(x)\left(\frac{v^{2}}{T^{*}}-\frac{3}{2}\right)\right)
$$




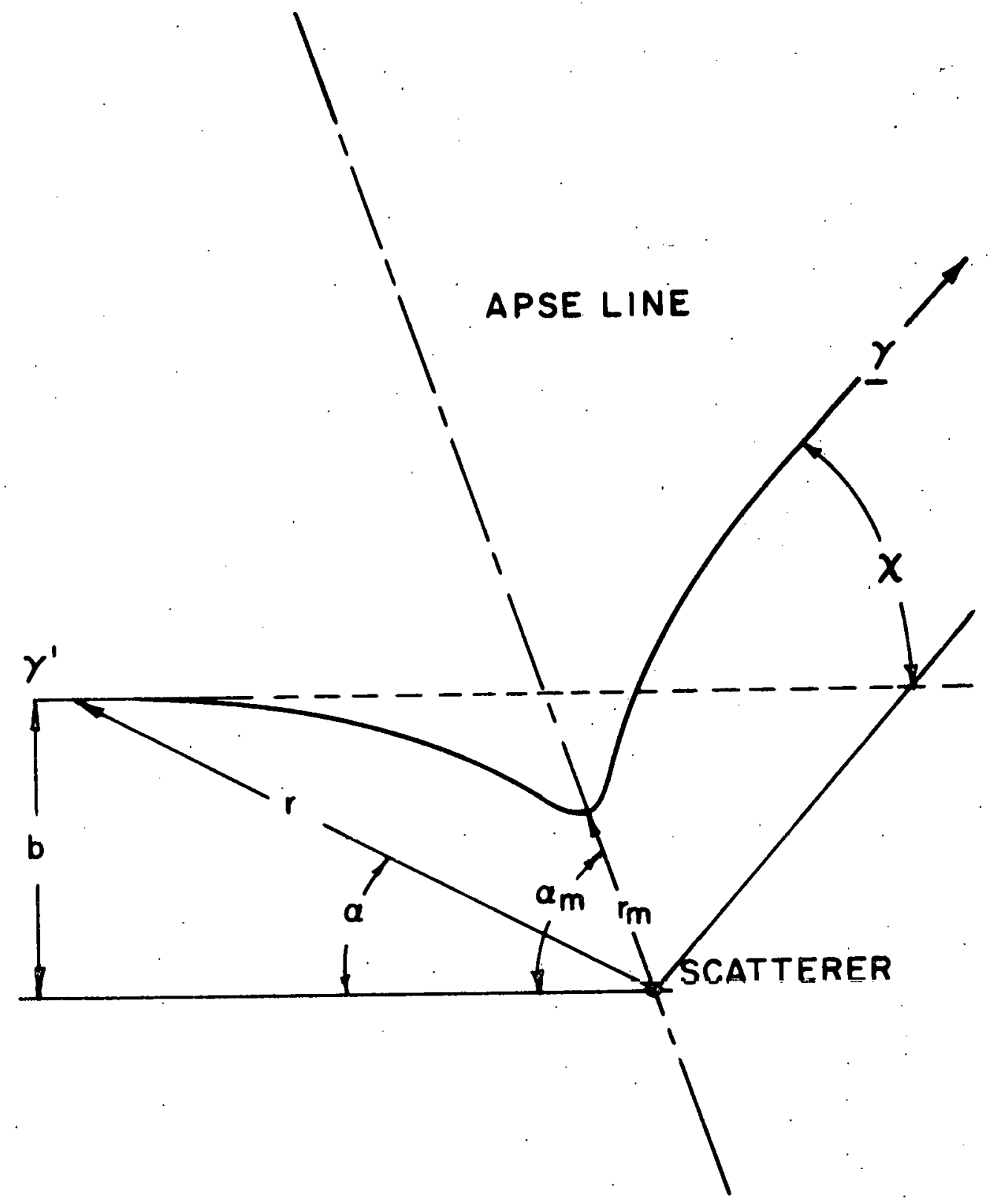

Figure 5.1 Dynamics of one dimensional collision 


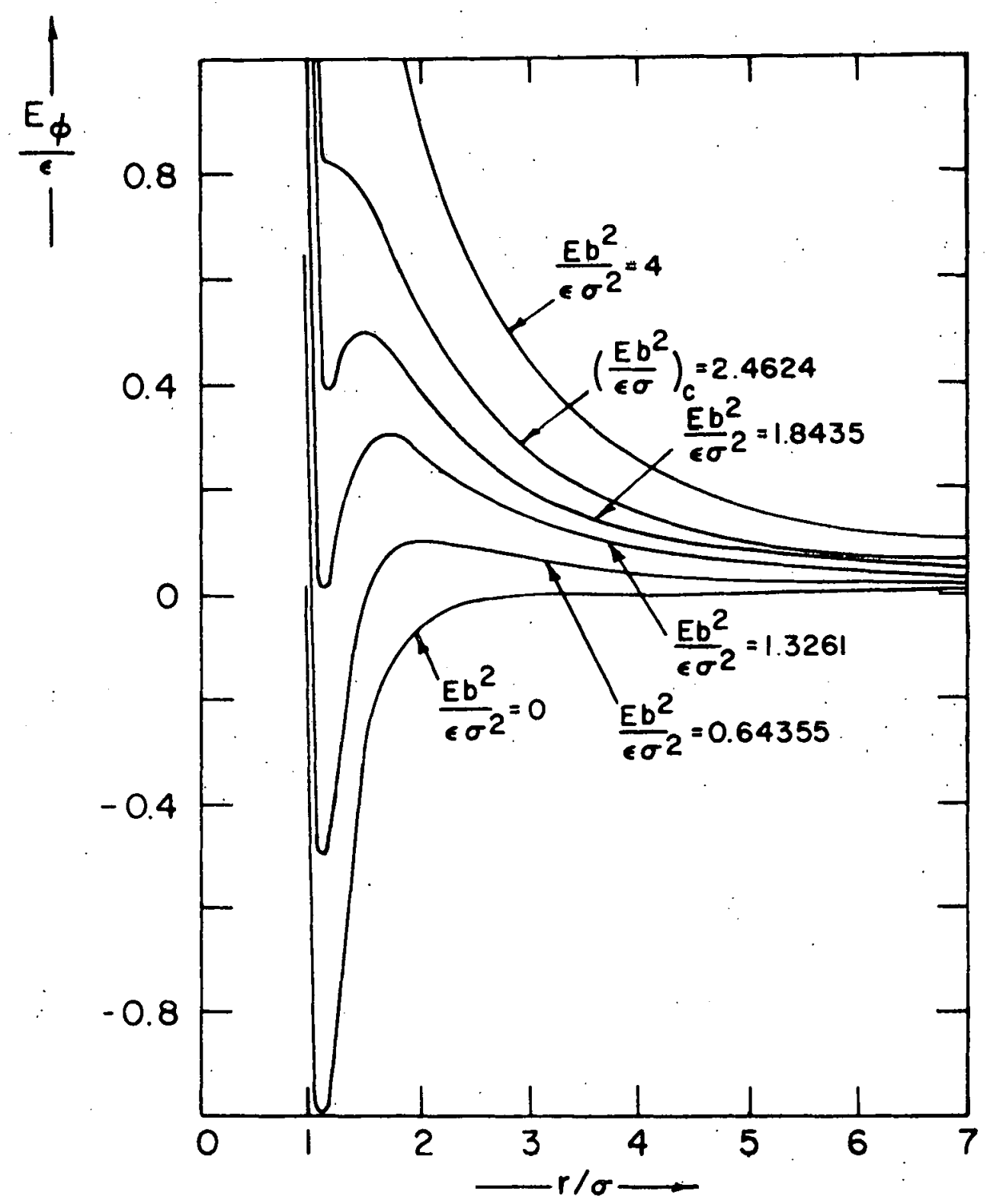

Figure 5.2 Effective potential curves for different values of the angular momentum (36) 
94

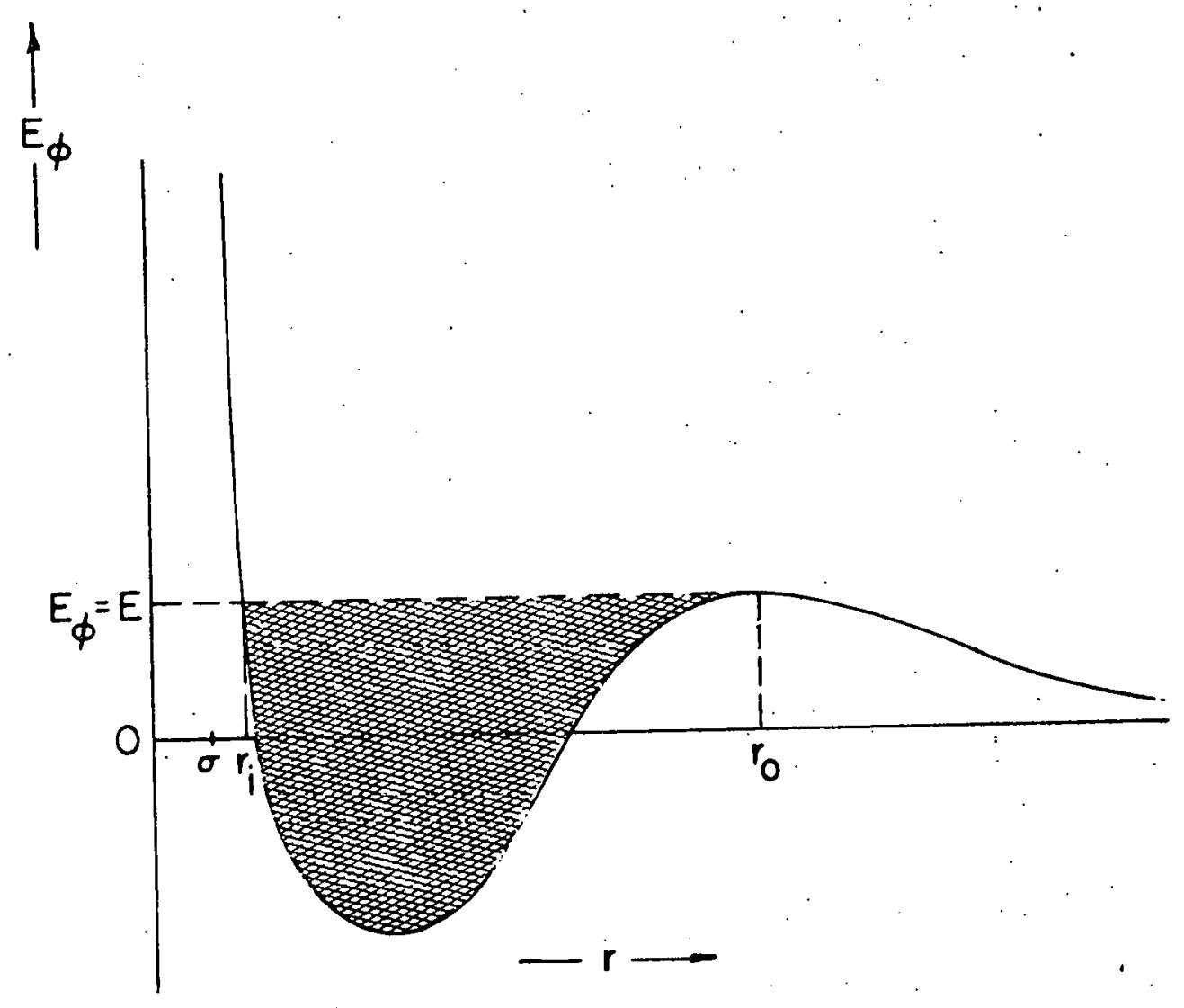

Figure 5.3 The effective potential for $\left(E b^{2}\right)<\left(E b^{2}\right)_{C}$ 
95

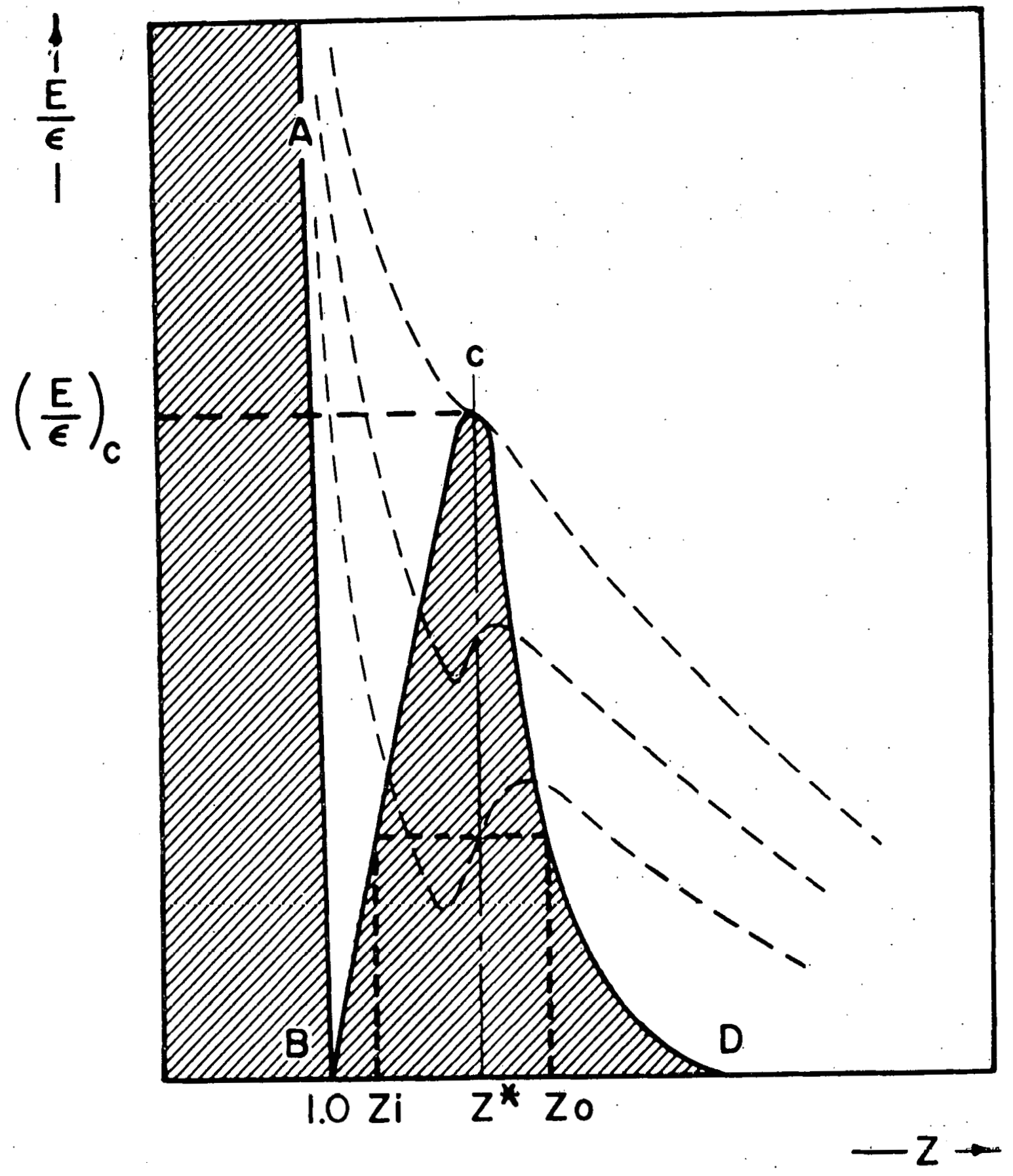

Figure 5.4 The region of integration $\left(\frac{E}{E}, z\right)$ 


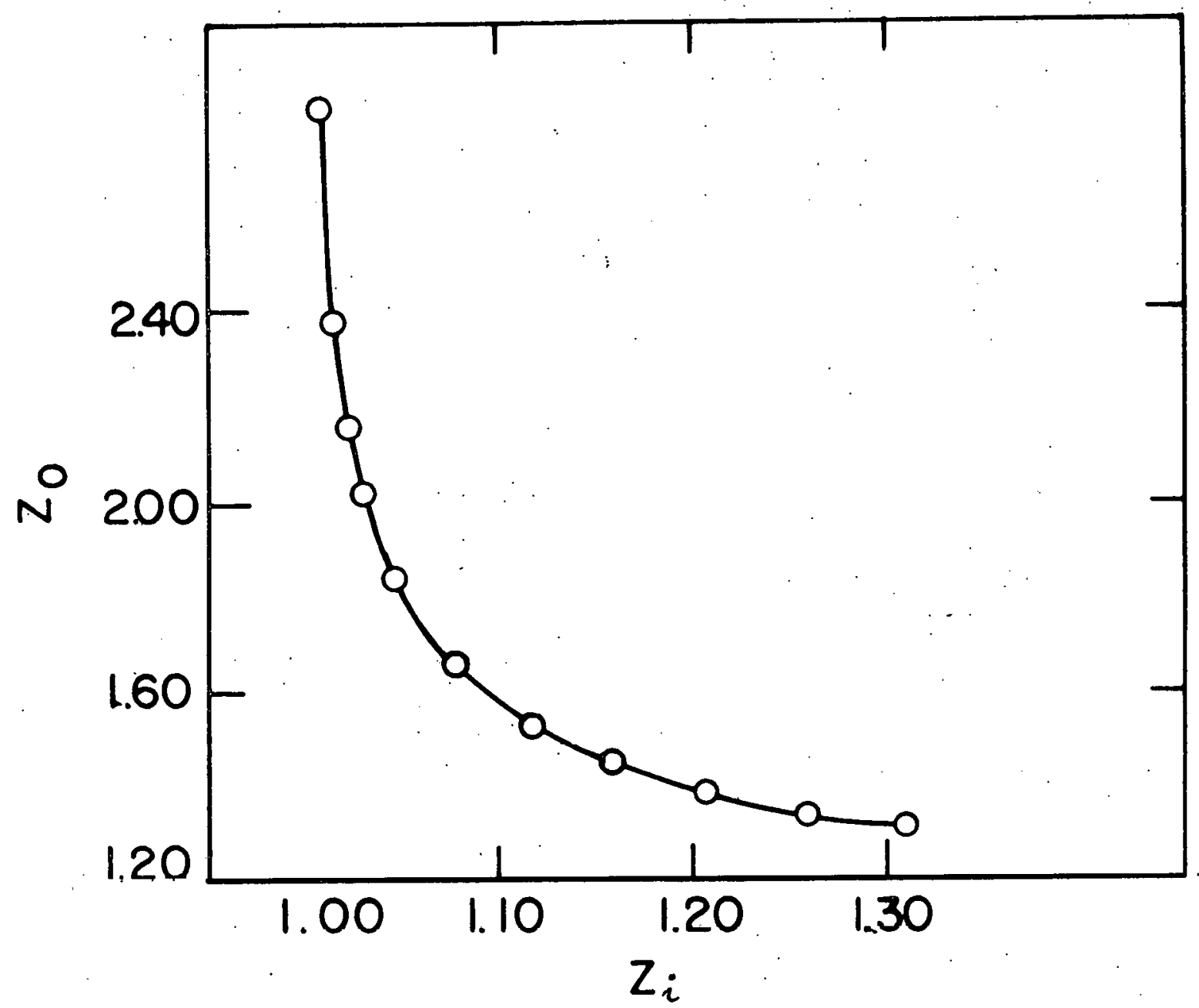

Figure 5.5 Representation of $z_{0}$ as a function of $z_{i}$ 


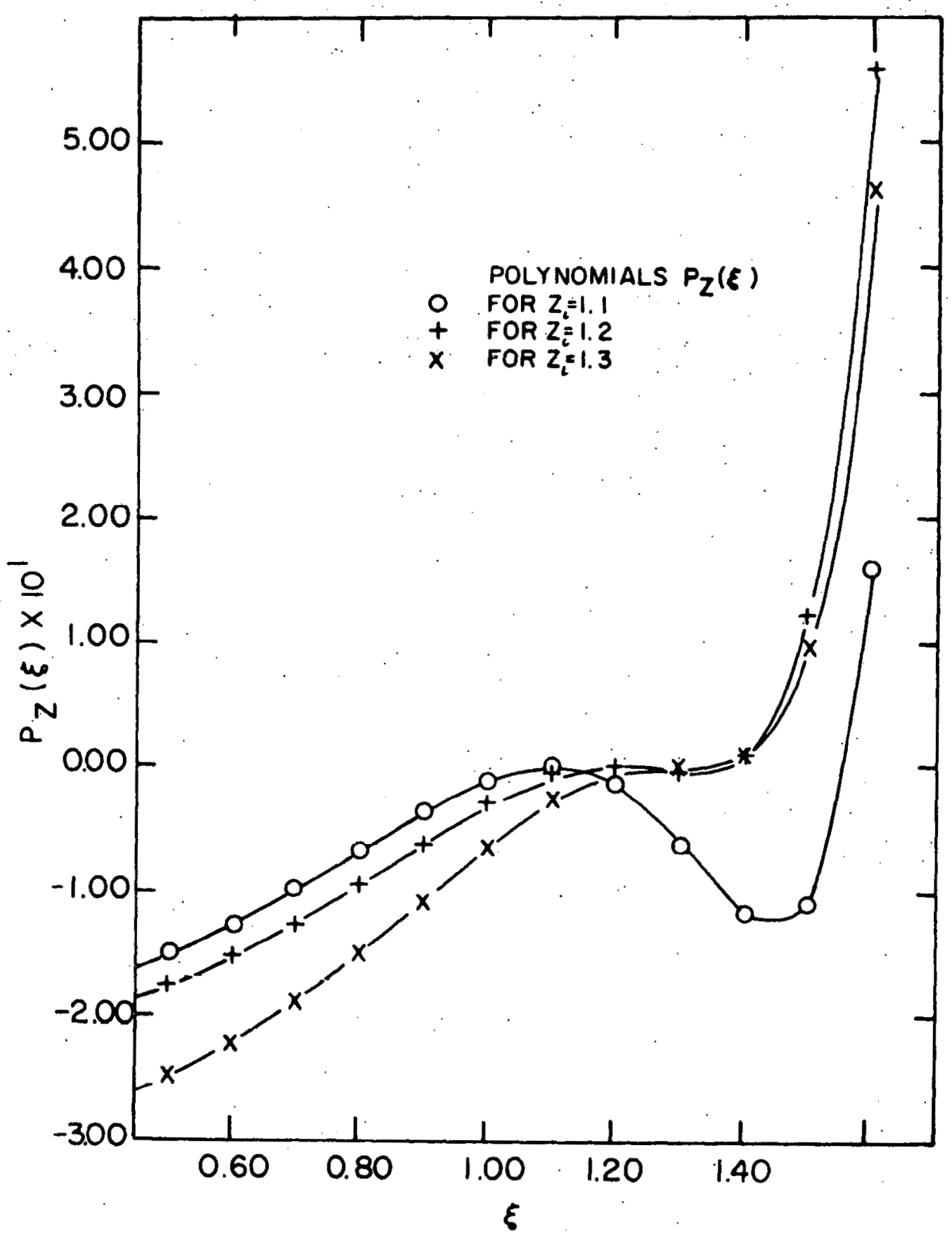

Figure 5.6 The twelfth degree polynomial $P_{z}$, for three different values of $z_{*}, 1<z_{c}<z^{*}$ 


\section{NUMERICAL RESULTS AND CONCLUSIONS}

\subsection{The Square Well Calculations}

For the square weil calculations, the integrations in Eq. (4.45) are limited to regions of the pre-collision relative velocity $\underline{\gamma}^{\prime}$ and of the impact parameter $b$ as represented in Fig. (6.1). Regions $I$ and II contain values of $b$ and $\gamma^{\prime}$ for collisions in which the two hard cores of the molecules touch. On the region III trajectories, only soft potential interactions occur. In Fig. (6.1) we note the existence of a critical value $\gamma_{c}^{\prime \prime}$ in the domain of $\gamma^{\prime \prime}$ such that for any $\gamma^{\prime \prime}<\gamma_{c}^{\prime}$ and $b<c$ (i.e., region $I)$ there is a hard interaction. This follows since, from the definition of $h\left(\gamma^{\prime}\right)$, we know that as $h \rightarrow c, \gamma^{\prime} \rightarrow \gamma_{c}^{\prime}$. On the other hand, as $h \rightarrow a, \gamma^{\prime} \rightarrow \infty$, the value of $\gamma_{c}$ is found from momentum conservation arguments to be

$$
\begin{array}{ll}
c \gamma^{\prime} & =\gamma a=a\left(\gamma_{c}^{\prime}{ }^{2}+\epsilon / k T\right)^{\frac{1}{2}}
\end{array}
$$

A transformation of variables is needed to rewrite Eq. (4.45) in a form more suitable for numerical solution.

If we let $t=\gamma^{2}$, then from Eq. (4.45) the five basic integrals that must be evaluated to find $I_{\eta}=\sum_{i=1}^{5} L_{\eta}^{(i)}$ transform to

$$
\begin{aligned}
& L_{\eta}^{(1)}=\int_{0}^{\infty} d t t \theta^{-t}\left(t+T^{*-1}\right)^{3 / 2} \\
& L_{\eta}^{(2)}=\int_{0}^{\infty} d t t^{\frac{3}{2}} \theta^{-t} \int_{0}^{h(t)} d b b c_{2}\left(\hat{\gamma}^{\prime} \cdot \hat{\underline{a}}\right) \\
& L_{\eta}^{(3)}=\int_{0}^{\infty} d t t^{\frac{3}{2}} \theta^{-t} \int_{0}^{h(t)} d b b c_{3}\left(\underline{\gamma}^{\prime} \cdot \underline{\hat{r}}_{0}\right)
\end{aligned}
$$




$$
\begin{aligned}
& I_{\eta}^{(4)}=\int_{0}^{\infty} d t t^{3 / 2} \theta^{-t} \int_{n}^{c} d b \cdot b a_{2}\left(\underline{\beta}^{\prime} \cdot \underline{n}\right) \\
& L_{\eta}^{(s)}=\int_{0}^{\infty} d t t^{1 / 2} \theta^{-t} \int_{0}^{h} d b b a_{1}\left(\underline{\beta_{1}} \cdot \underline{\hat{c}}\right)
\end{aligned}
$$

The vectors $\underline{a}, \underline{r}_{0}$ and $\underline{c}$ were introduced in chapter 4 (Figs. $(4.2,4.3))$. After some numerical experimentation, it was found that the Gauss and Gauss-Laguerre 32 points quadrature formulae give the best results in the ovaluation of both I.y and $I_{\boldsymbol{\lambda}}(40)$. Accuracy tests were performed for these calculations by alternatively changing the number of quadrature points of one integration while keeping the quadrature points of the other constant. The results of these tests for the evaluation of $I_{\lambda}$ for argon are given in the following table:

Table 6.1 Gauss-Laguerre and Gauss approximations of . $I_{\lambda}$ for Argon

\begin{tabular}{cccc}
\hline$I_{\lambda}$ & $\mathrm{DQGn}$ & $I_{\lambda}$ & $\mathrm{DQAn}$ \\
\hline 65.903006 & 8 & 66.195635 & 8 \\
65.904911 & 16 & 66.001000 & 16 \\
65.905185 & 32 & 65.938435 & 24 \\
65.905182 & 64 & 65.905185 & 32 \\
\hline
\end{tabular}

The symbols DQGn and DQAn are the abbreviations for the IBM Scientific Subroutine Package which executes the n-points Gauss and Gauss-Laguerre method of integration on the IBM model $360 / 65$ computer. The computation time for one value of $\mathrm{T}^{*}$ was $13.16 \mathrm{sec}$. for $I_{\lambda}$ and $13.39 \mathrm{sec}$. for $I_{\eta}(41)$. 
6.2 The Lennard-Jones Calculation (37)(38)

For the Lennard-Jones calculation, the integration over $v$ and $z$ in $I_{\eta}$ and $I_{\lambda}$, must be limited to the nonbound state region, which is the nonshaded area in Fig. (5.4). For computational convenience we decompose I into three parts corresponding to the three possible limits of integration for $\mathrm{z}$ and $\mathrm{v}$ as follows:

$$
\begin{aligned}
I_{\eta}=\sum_{i=1}^{3} I_{\eta}^{(i)}, I_{\eta}^{(i)}= & a\left(T^{*}\right) \int_{v_{(i)}}^{\infty} d v \int_{z \ell_{(i)}}^{2 u(i)} d z \int_{z}^{\infty} d x x^{3} v^{3} z F^{\prime}(x) e^{-v^{2} / T^{*}} \\
& (A(x, v, z) H(v, z) / G(x, v, z))
\end{aligned}
$$

where $\nabla_{(1)}=(F(z))^{1 / 2} \quad, z_{e_{1}}=0, z_{u_{1}}=1$

$$
\begin{aligned}
& v_{(2)}=\left(F(z)+\frac{z_{0}^{3}}{g z} F^{\prime}\left(z_{0}\right)\right)^{1 / 2}, z_{e_{2}}=1, z_{u_{2}}=z^{*} \\
& v(3)=\left(F(z)+\frac{z}{2} F^{\prime}(z)\right)^{1 / 2}, \quad z_{e_{3}}=z ; \quad z_{u_{3}}=\infty
\end{aligned}
$$

To find $A(x, v, z)$, we must know $\alpha$ which is defined by Eq. (5.18). In Eq. (5.18) we replace $y$ by the variable w which is defined by the transformation

$$
w=(y-3 x) /(y+x) \quad-1 \leq w \leq 1
$$

Since the integration limits on $w$ are fintte we can use Gauss's quadrature formula (eight points are required) to compute $\alpha$.

The integrations over $x$ are complicated by the existence of a pole at the lower limit of integration, since as $x \rightarrow z, G(x, z, v) \rightarrow 0$. However, of course, the integral exists. In fact, when $x=2$, the internuclear separation of the colliding molecules is equal to their distance of closest approach. 
At this point the radial component of the relative velocity vanishes and consequently the total energy $E$ is equal to the effective potential $E_{\phi}$. Therefore, as $x \rightarrow z, H(x, v) \rightarrow 0$ which assures the convergence of $I_{\eta}$.

In order to use a Gauss-Laguerre quadrature formula for the $x$ integration we introduce the change of variable

$$
t=(x-3 z) /(x+z) \quad-1 \leq t \leq 1
$$

I'o guarantee sufficient accuracy with a minimum of computation time it'is necessary to subdivide the range of $t$ into five intervals. Sixteen point formulae were used in four intervals and a 32 point formula was used in the fifth. These guaranteed an accuracy of four significant figures.

In $I_{\eta}$, the variable $v$ is replaced by $l$ with

$$
l=\left(v-3 v_{(i)}\right) /\left(v+v_{(i)}\right) \quad-1 \leq \ell \leq 1
$$

Note that as $e \rightarrow+1$, the integrand in $I_{\eta}$ has the indeterminant form 0/0. The exponential $e^{-v^{2} / 4 x}$ makes the numerator converge to zero faster than the demominator so that as $l \rightarrow+1, I_{\eta} \rightarrow 0$. However, in order to avoid computational difficulties, the upper limit of integration was replaced by $1-10^{-6}$. In $I_{\eta}^{(1)}$, if we make the substitution (37)

$$
s=\left(T(z) / T^{*}\right)((3 i e) /(1-e))^{2}, 0 \leqslant 3<\infty
$$

then the integrand $I_{\eta}^{(1)}$ transforms to $I_{\eta}^{\prime \prime \prime}=\ldots \int \mathrm{d} s \ldots e^{-s}$ 
and the s-integration can be performed with an eight point Gauss-Laguerre quadrature formula with great accuracy. In $I_{\eta}^{\prime \prime \prime}$ the integration over $\mathrm{z}$ can be executed immediately with a 32 point Gauss's formula; the integrations in $I_{\eta}^{(2)}$ are similar to the ones in $I_{\eta}^{(1)}$. In $I_{\eta}^{(3)}$ we perform first the change of variablo

$$
m=\left(z-3 z^{*}\right) /\left(z+z^{*}\right) \cdot-1 \leq m \leq 1
$$

and then use the same quadrature formulae. A summary of the integration methods used in the computation of $I_{\eta}$ is given in table 6.2.

Table 6.2 Quadrature formulae chosen in the computation of $I_{\eta}$

\begin{tabular}{cccc}
\hline & $I_{\eta}^{(1)}$ & $I_{\eta}^{(2)}$ & $I_{\eta}^{(3)}$ \\
\hline$l$ & DQG8 & DQG16 & DQG8 \\
$\mathbf{s}$ & DQL8 & - & - \\
$z$ & -- & DQG32 & -- \\
$\mathrm{m}$ & -- & -- & DQG32 \\
$\mathrm{t}$ & DQG96 & DQG96 & DQG96 \\
w & DQG8 & DQG8 & DQG8 \\
\hline
\end{tabular}

A more detailed discussion of the techniques used in the computation of $I_{\eta}$ and $I_{\lambda}$ as well as a listing of the explicit forms of $\left(I_{\eta}^{(i)}\right)_{i=1}^{3}$ used in the fortran program can be found in refer- 
ence (37). In this same reference, the accuracy of each quadrature used is given, and the results of the tests are presented in tabular form. A fortran double precision program for $I_{\lambda}$ can be obtained from the one for $I_{\eta}$ after some simple modifications. The computation time for one value of $T^{*}$ is of 339.81 secs. for $I_{y y}$ and 61.41 secs. for $I_{\lambda}$.

\subsection{Numerical Results}

We now compare transport coefficients calculated from our theory using the square well potential and the LennardJones potential with those obtained from experimental data. The Lennard-Jones potential is somewhat more realistic than the square well potential in that it is continuously varying; on the other hand, the square well potential is a three paramors potential and hence more flexible. Both potentials are in qualitative agreement with experimentally determined interaction potentials in that they are attractive at large intermolecular separations and repulsive at short separations. Comparison of calculated results using the two forms of the potential illustrates the sensitivity of the transport coefficients to the detailed form of the interactions. 'The calculated results using the two forms are not in quantitative agreement, but they do show the same qualitative temperature dependence. This qualitative behavior is in agreement with experiment; however, the experimental data is not sufficiently 
reliable to make quantitative comparisons meaningful.

The square well potential leads to transport coefficients which are universal functions of the reduced temperature $T$ and of the ratio $D=a / c$, therefore all gases with the same $D$ should obey a law of corresponding states for a plot of $B_{\eta}^{*}$ or $B_{\lambda}^{*}$ vs. $T^{*}$. In tables 6.4 and 6.5 and Figs. $(6.2,6.3,6.4,6.5)$ the results of the square well calculaion are given. Table 6.3 below, contains the parameters used in the calculations.

Table 6.3 Parameters of the square well model for various gases as found from viscosity (42)

\begin{tabular}{lcccc}
\hline & $c / K(K)$ & $a(\AA)$ & $c(\AA)$ & $D$ \\
\hline $\mathrm{N}$ & 80 & 3.36 & 7.00 & 0.480 \\
$\mathrm{CO}$ & 91 & 3.29 & 7.48 & 0.440 \\
$\mathrm{H}$ & 94 & 2.57 & 3.67 & 0.700 \\
$\mathrm{O}$ & 94 & 3.16 & 6.72 & 0.470 \\
$\mathrm{Ne}$ & 101 & 2.38 & 3.66 & 0.650 \\
$\mathrm{~A}$ & 167 & 2.98 & 5.84 & 0.510 \\
$\mathrm{CH}$ & 174 & 3.35 & 6.57 & 0.510 \\
$\mathrm{H} \theta$ & 232 & 1.90 & 2.64 & 0.720 \\
$\mathrm{Air}$ & 87 & 3.30 & 6.88 & 0.480 \\
\hline
\end{tabular}

The poor agreement between the experimental and calculated $\beta_{\lambda}^{*}$ is not surprising since we did not use thermal conductivity 
parameters, Fig. (6.4). Holleran and Hulburt (42) noted that different sets of parameters for the same gases are needed for each transport coefficient to reproduce experimental data. For example; for neon they reported that $D=0.65$ and $D=0.55$ give good results respectively in the case of the viscosity and themal diffusion coefficients. In Fig. (6.4) we can verify the findings of Holleran and Hulburt. If the viscosity parameter $D=0.51$ of argon is replaced by $D=0.72$, the parameter of helium, we obtain good agreement with experiment. An interesting feature of the $B_{i}^{*}$ data, seo table 6.5, is that all curves of $B_{\eta}^{*}$ vs. $T^{*}$ reach a minimum with $\beta_{j}^{*}\left\langle 0\right.$ and $T^{*}>10$ before approaching zero. A curve with this shape, was first obtained by Stogryn and Hirshfelder (36) and will be discussed later.

Density corrections to the transport coefficients of the Lennard-Jones gas are of particular interest in that they have been previously computed using different theories. Iney can be written in the form

$$
\begin{aligned}
& B_{\eta}^{*}=b_{\eta}\left(T^{*}\right)+I_{\eta}\left(T^{*}\right)=b_{\eta}\left(T^{*}\right)+\sum_{L=1}^{3} I_{\eta}^{(i)} \\
& B_{\lambda}^{*}=b_{\lambda}\left(T^{*}\right)+I_{\lambda}\left(T^{*}\right)=b_{\lambda}\left(T^{*}\right)-\frac{2}{3} I_{\eta}\left(T^{*}\right)+\sum_{L=1}^{3} I_{\lambda}^{(i)} \\
& b_{\eta}\left(T^{*}\right)=\frac{4 \pi}{15}\left(B^{*}+T^{* *}\right), B^{*}=3 B / 2 \pi \sigma^{3} \\
& b_{\lambda}\left(T^{*}\right)=\frac{2}{5} \pi\left(B^{*}-\frac{5}{9} T^{*^{2}} B^{*}-\frac{7}{9} T^{*^{*} * *} B^{*}\right)
\end{aligned}
$$

where

The values of these functions are given in tables 6.6 and 6.7 and are represented graphically in Figs. $(6.6,6.7) ; I_{\eta}$ and 
$I_{\lambda}$ have already been defined in chapter 5 , and $B, \dot{B}$, and $\ddot{B}$ in chapter 3. With Figs. $(6.6,6.7)$ we can compare the relative contribution of $b_{\eta}$ and $I_{\eta}, b_{\lambda}$ and $I_{\lambda}$, to the respective transport coefficients. The functions $b_{\eta}$ and $b_{\lambda}$ contain the contributions to $B_{\eta}^{*}$ and $B_{7}^{*}$ due to correlated velocities and temperatures.

In Figs. $(6.8,6.9)$ we compare our results with experiment and with the work of Stogryn and Hirshfelder (,$\left.H_{,}\right)$, Snider and Curtiss (S.C.), and Hoffman and Curtiss (H.C.). In Fig. (6.8) the experimental points in circles have been given by Stogryn and Hirshfelder, the points in squares by Flynn, Hanks, Lemaire and Ross (43). In Fig. (6.8) the two points in squares are values given by Sengers, Bolk and Stigter (44). In Fig. (6.8), the curve of Snider and Curtiss represents first density corrections due to collisional transfer contributions only. Hoffman and Curtiss improved the curves of Snider and Curtiss by adding a correction due to three body collision contributions. In both works, the theory fails completely in the low temperature region. Of particular interest is the resemblance of our curves with Stogryn and Hirshtelder's since the first density corrections of these authors include bound states (dimers) effects. Iheir work, like ours, ignored three body collisions.

Stogryn and Hirshfelder made a semi-theoretical calculation of the transport coefficients of a Lennard-Jones gas. 'They calculated collisional transfer contributions from 
Enskog's theory and dimers contributions by treating the gas as a mixture of monatomic and diatomic molecules. From their work, these authors concluded that: (1) the initial density dependence of the viscosity is due mainly to collisional transfer contribtions in both the low and high temperature regions, (2) the initial density dependence of the thermal conductivity is due mainly to collisional transfer contributions only in the high temperature regions, while in the low temperature regions it is the bound molecules (diamers) contributions which predominate.

The theoretical calculations of Snider and Curtiss, as well as our contradict the first of Stogryn and Hirshfelder conclusions, but are in complete agreement with the second. In Fig. (6.9), we note that the $B_{\lambda}^{*}$ curve, at high $T$, follows Snider and Curtiss's while in the low $T$ regions follows Stogryn and Hirshfelder!s.. Over the entire temperature range there is satisfactory agreement with experimental data implying that multibody collisions do not contribute appreciably to the first density correction of the coefficient of thermal conductivity.

Even if the considerable uncertainty. associated with measurements is taken into consideration $(18)$, our $\beta^{*}$ vs. $T^{*}$ curve in Fig. (6.8), is low with respect to experimental data. Futhermore, as $T^{*} \rightarrow \infty$, our curve approaches zero from above (positive values) in disagreement with Hoffman and Curtiss's 
results (and experiment). We conclude, therefore, that bound states and multibody collisions must contribute significantly to the viscosity virial cefficient. 


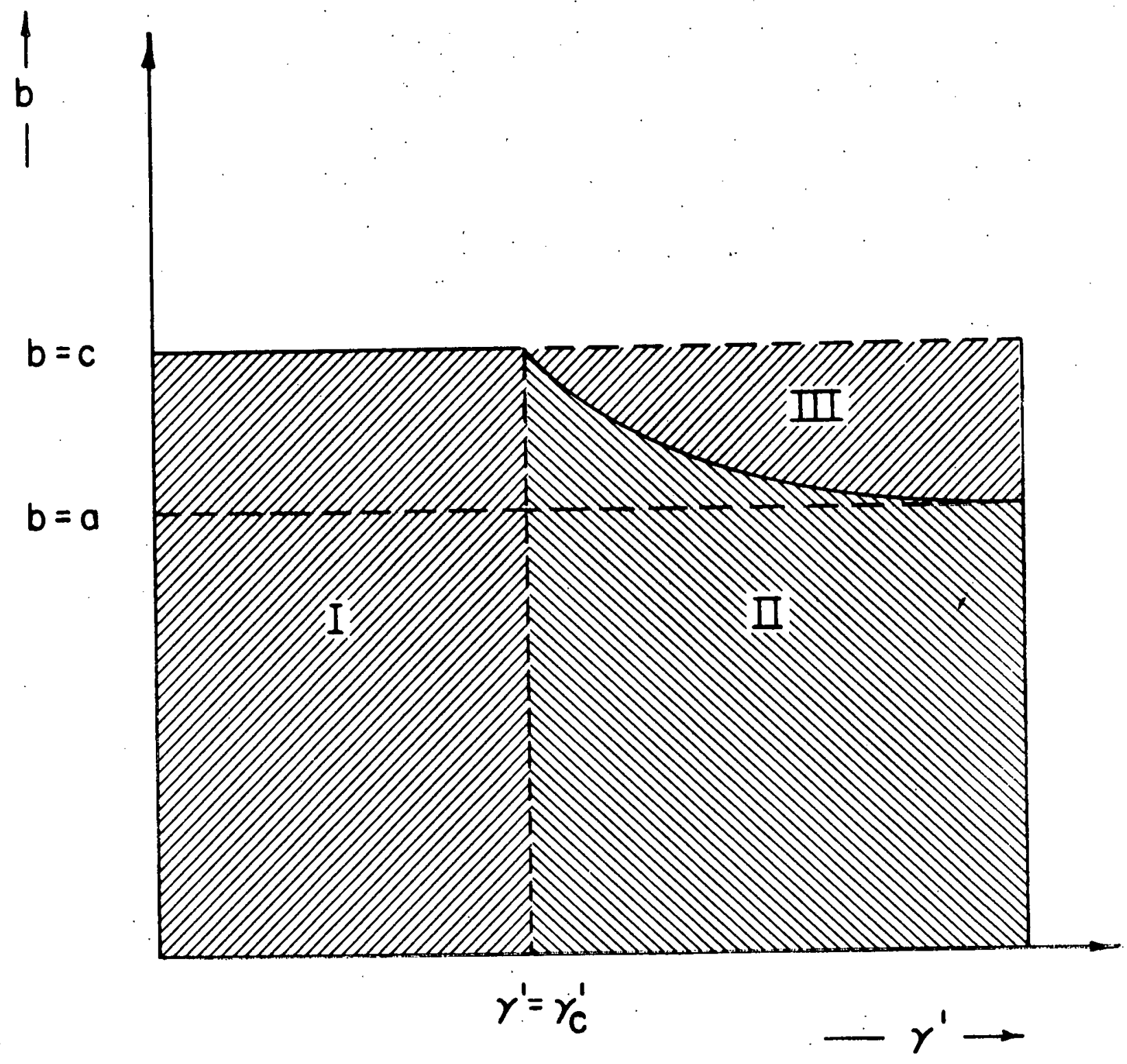

Figure 6.1 The region of integration $\left(b, \gamma^{\prime}\right)$ 


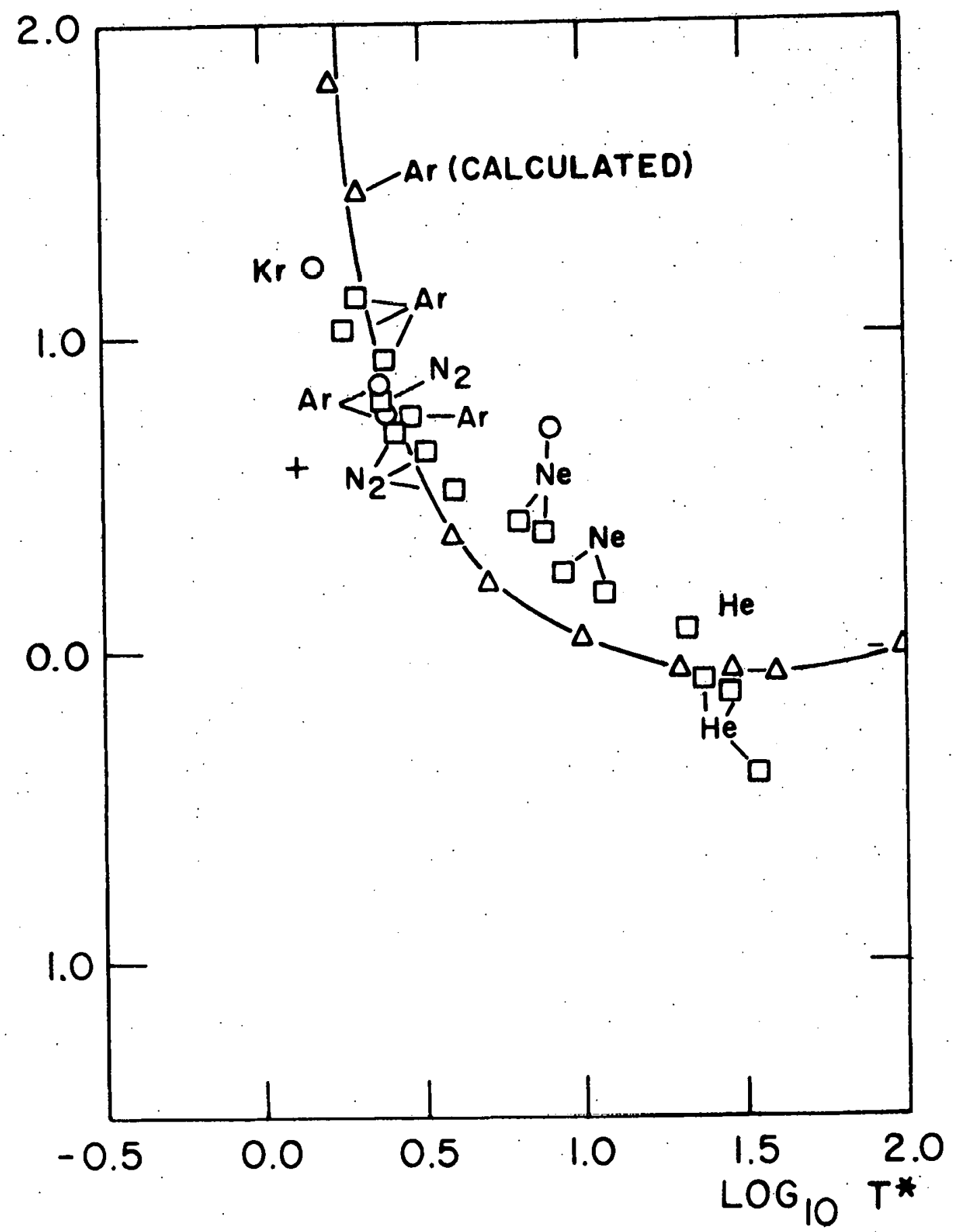

Figure 6.2 The square well, viscosity virial coefficient compared with experiment (36) 


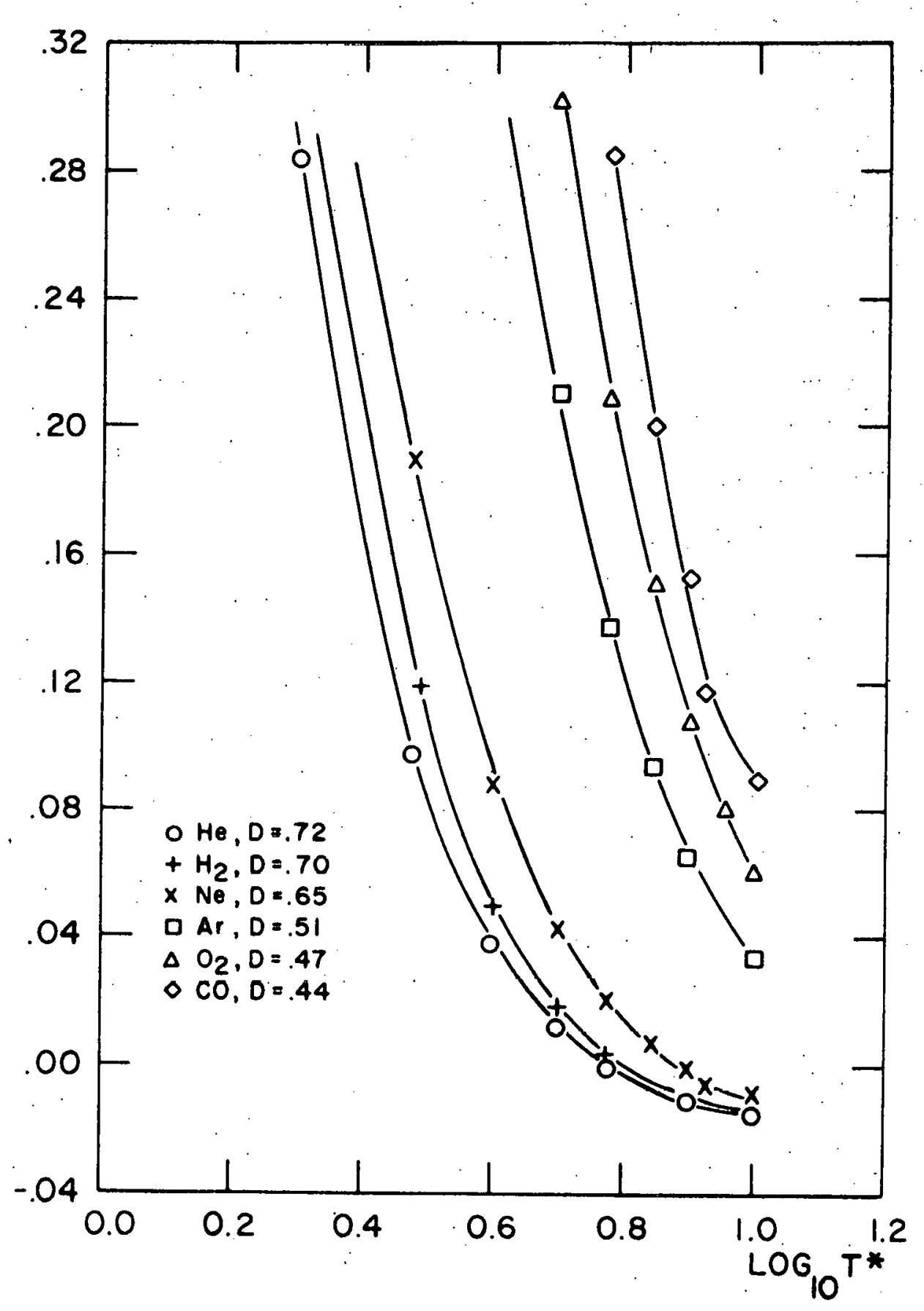

Figure 6.3. The square well, viscosity virial coefficient for different values of the parameter $D=a / c$ 


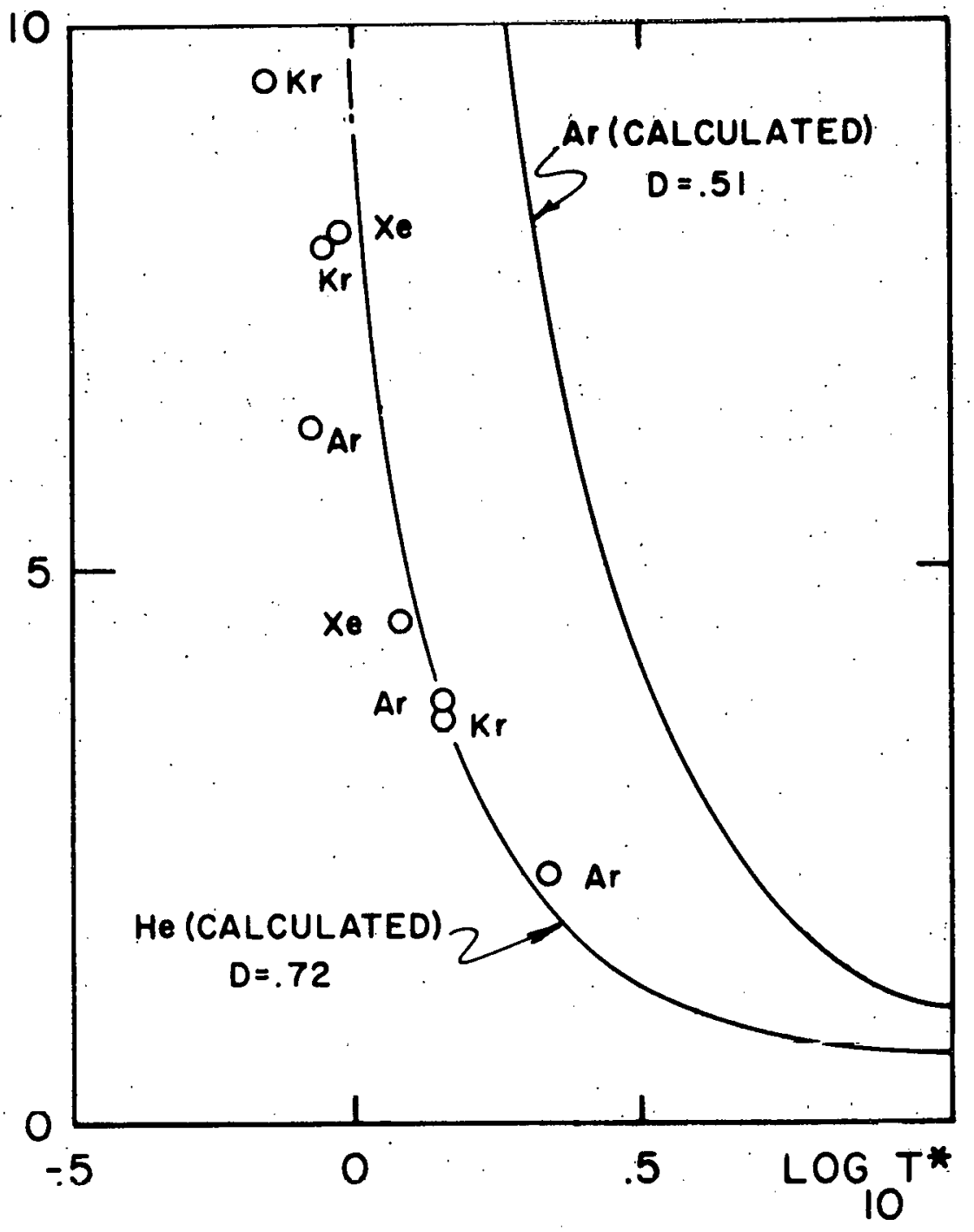

Figure 6.4 The square well, thermal conductivity virial coefficient, compared with experiment (44) 


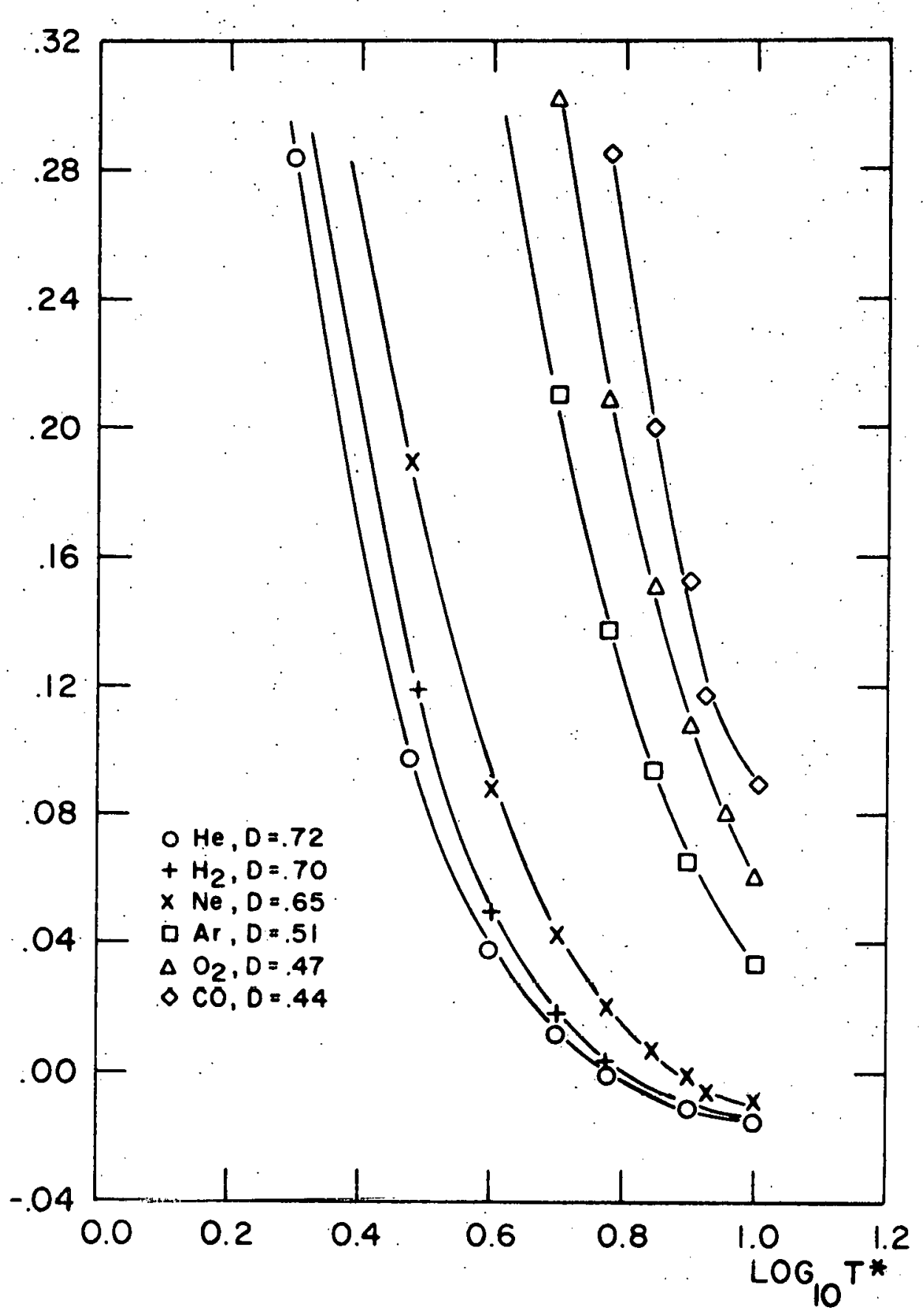

Figure 6.5 The square well, thermal conductivity virial coefficient for different values of $\mathrm{D}=\mathrm{a} / \mathrm{c}$ 


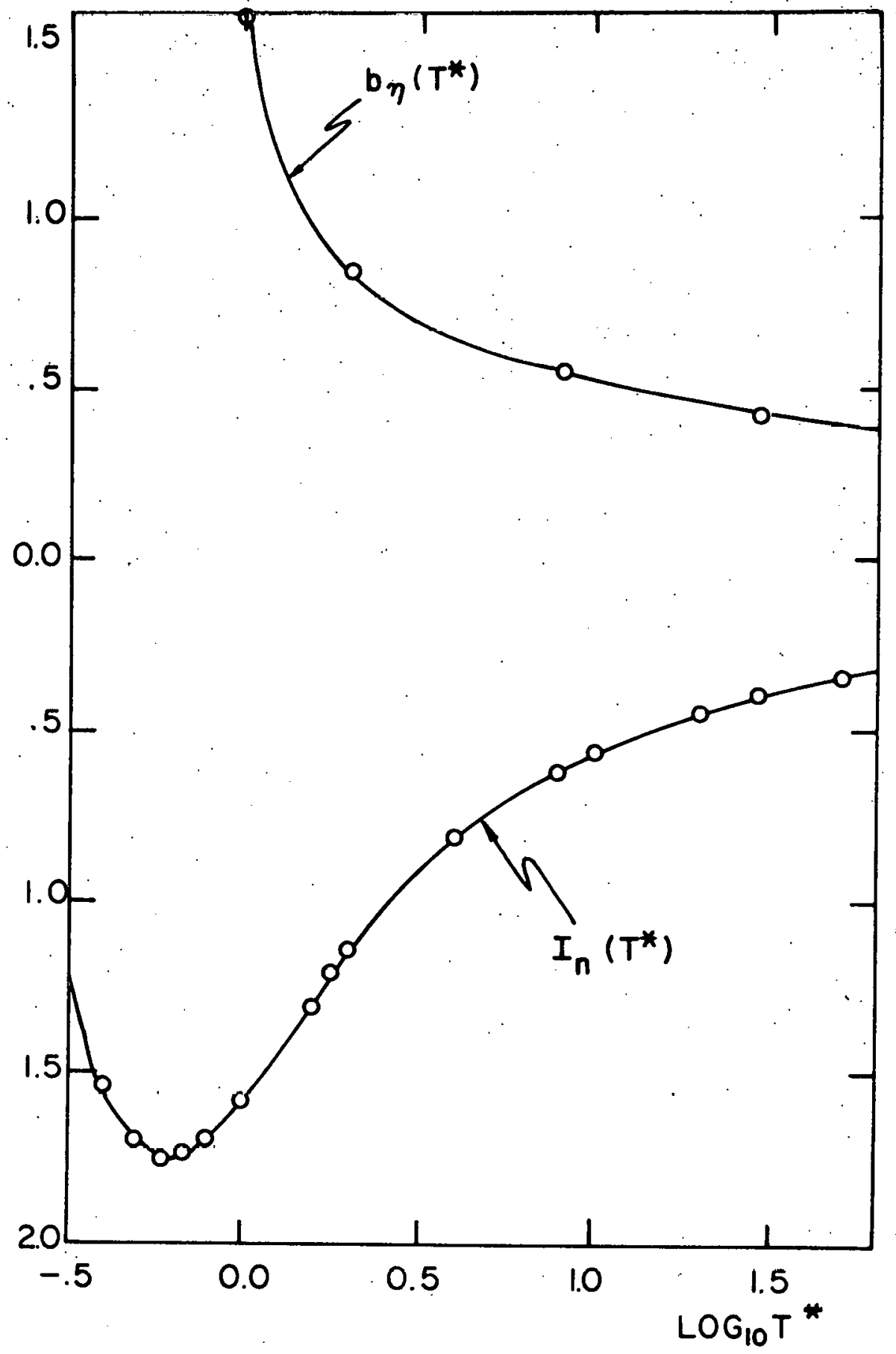

Figure 6.6 The functions $b_{\eta}\left(T^{*}\right)$ and $I_{\eta}\left(T^{*}\right)$ in $B_{\eta}\left(T^{*}\right)=b_{\eta}\left(T^{*}\right)$ $+I_{\eta}\left(T^{*}\right)$, the viscosity second virial coefficient 


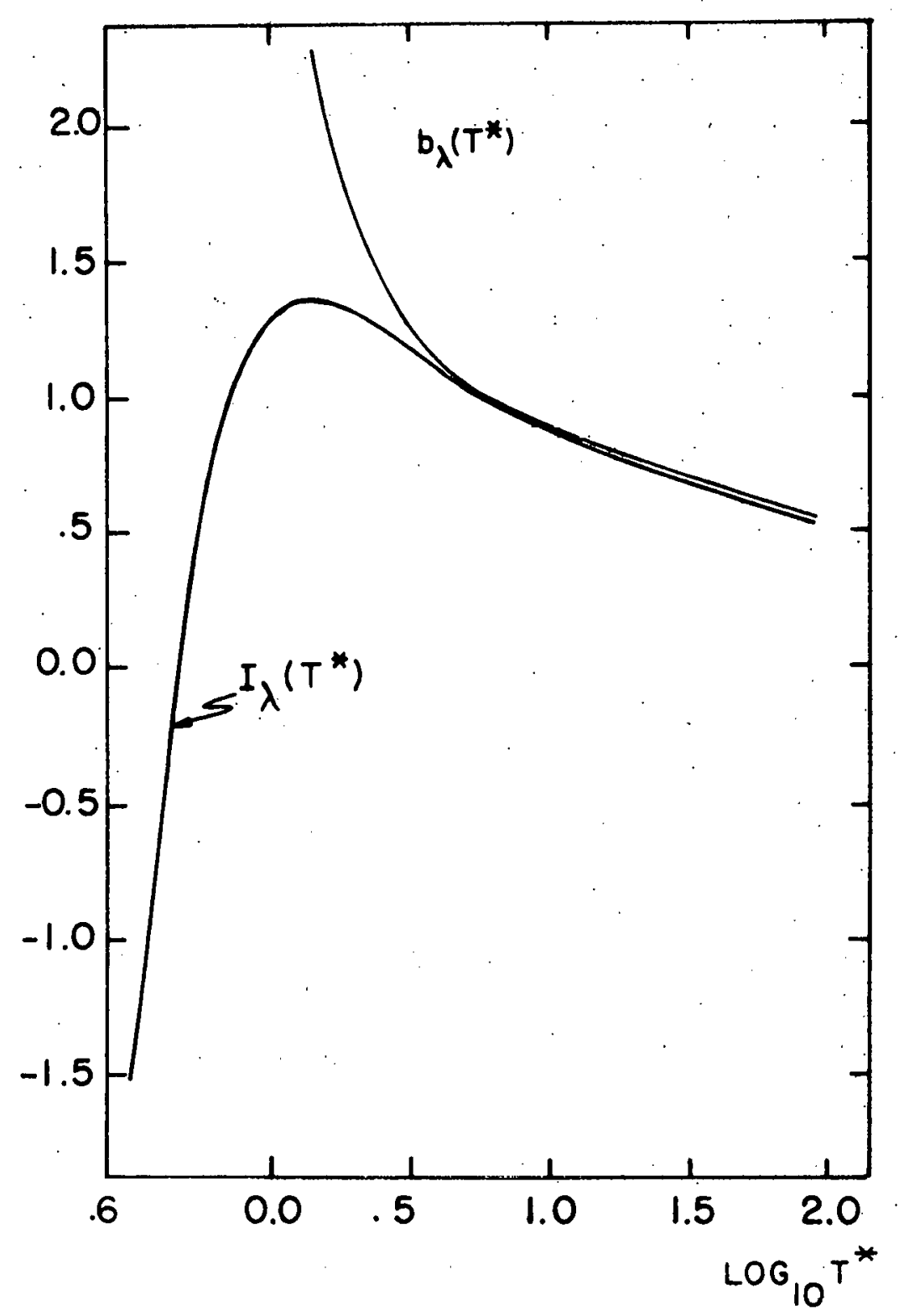

Figure 6.7 The functions $b_{\lambda}\left(T^{*}\right)$ and $I_{\lambda}\left(T^{*}\right)$ in $B_{\lambda}\left(T^{*}\right)=$ $b_{\lambda}\left(T^{*}\right)+I_{\lambda}\left(T^{*}\right)$, the thermal conductivity virial coofficient 


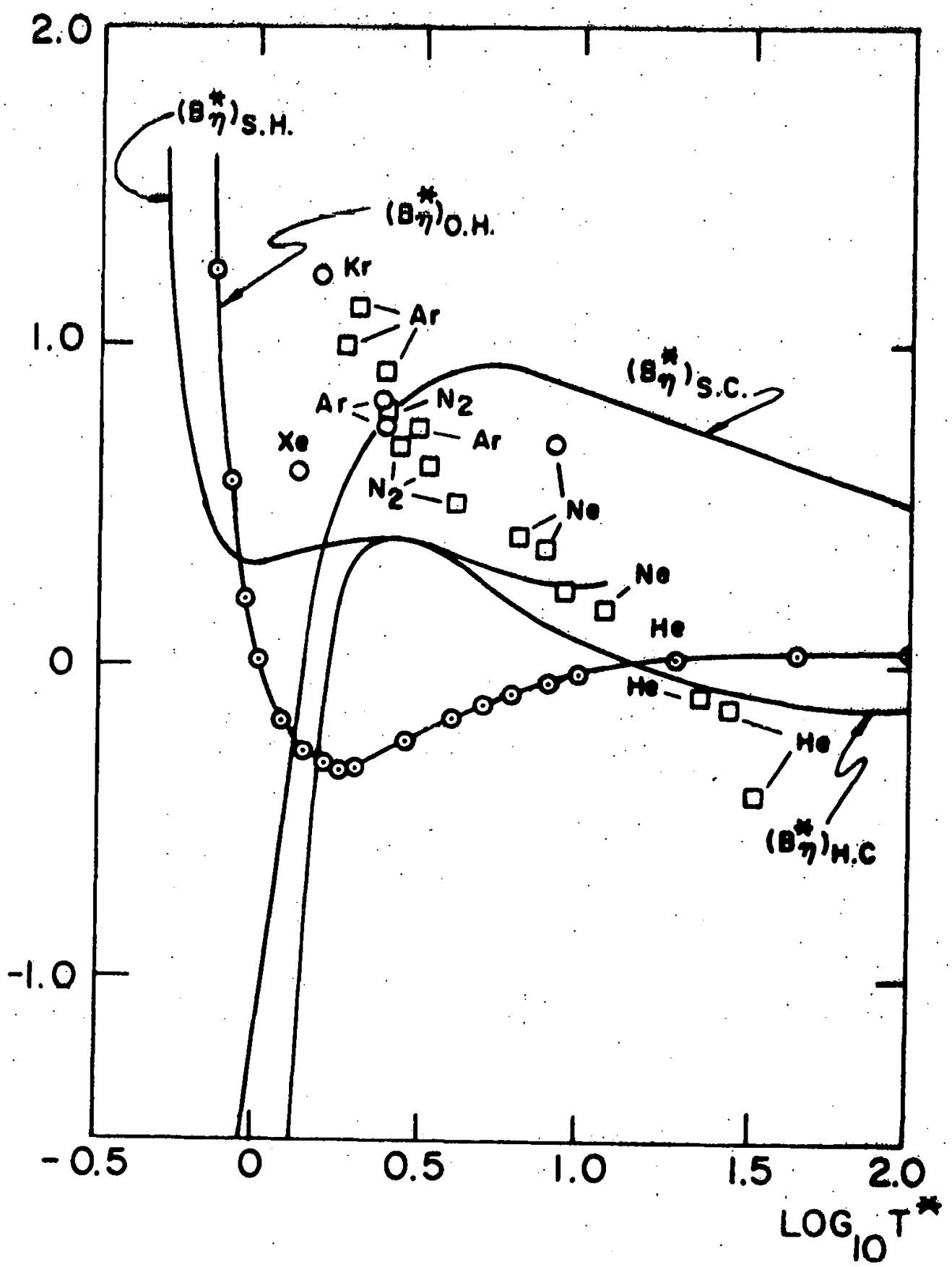

Figure 6.8 The viscosity second virial coefficient of Occelli and Hoffman (O.H.), compared with the results of Snider and Curtiss (S.C.), Hof fman and Curtiss (H.C.), Stogryn and Hirshfelder (S.H.), and experiment (36) (43) 


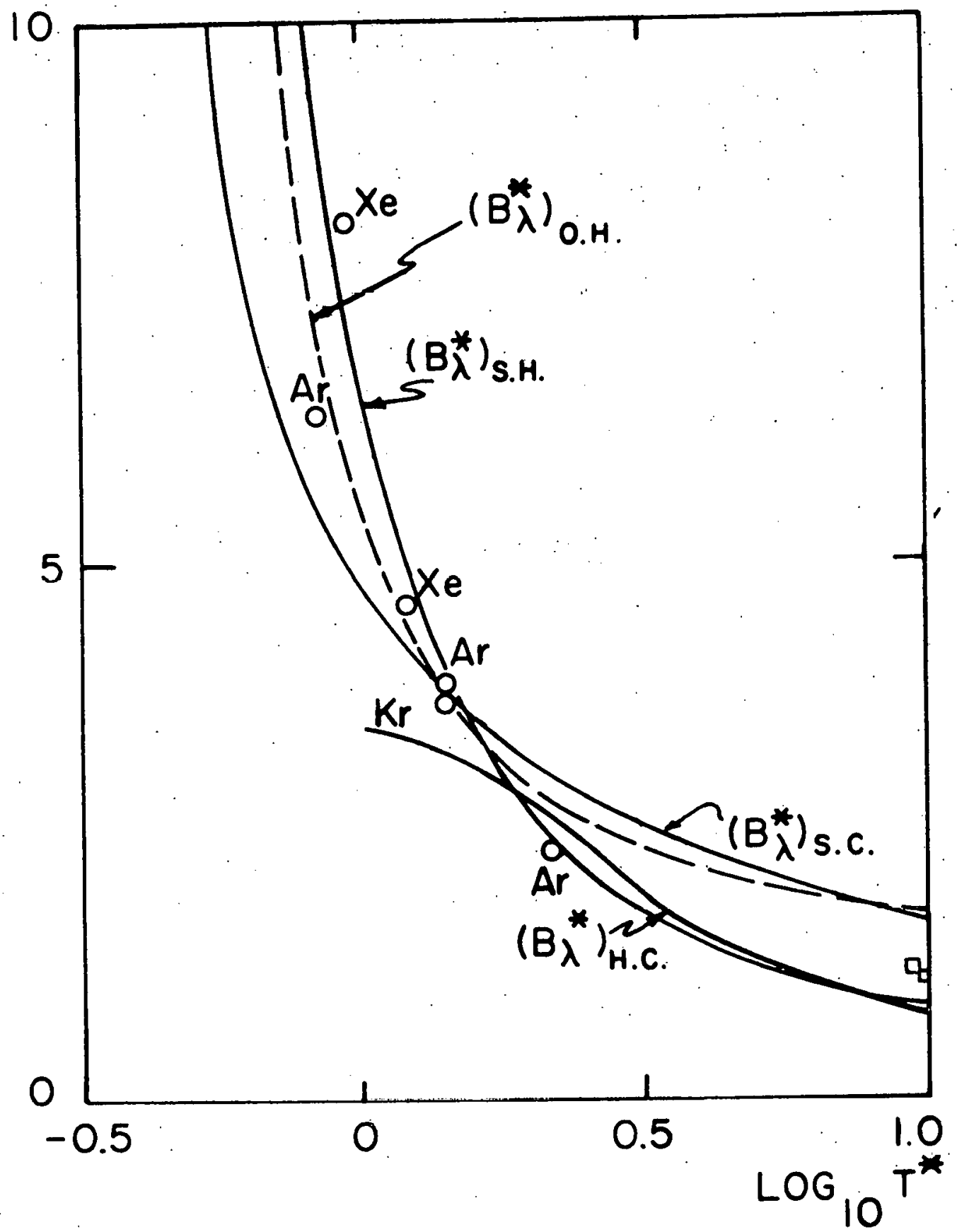

Figure 6.9 The thermal conductivity second virial coefficient of Occelli and Floffman (O.H.), compared with the results of Snider and Curtiss (S.C.), Hoffman and Curtiss (H.C.), Stogryn and Hirshfelder (S.H.) and experiment $(44)$ 
Table 6.4 The thermal conductivity virial coefficient $\beta_{\lambda}\left(\mathrm{T}^{*}\right)$ for the square well model

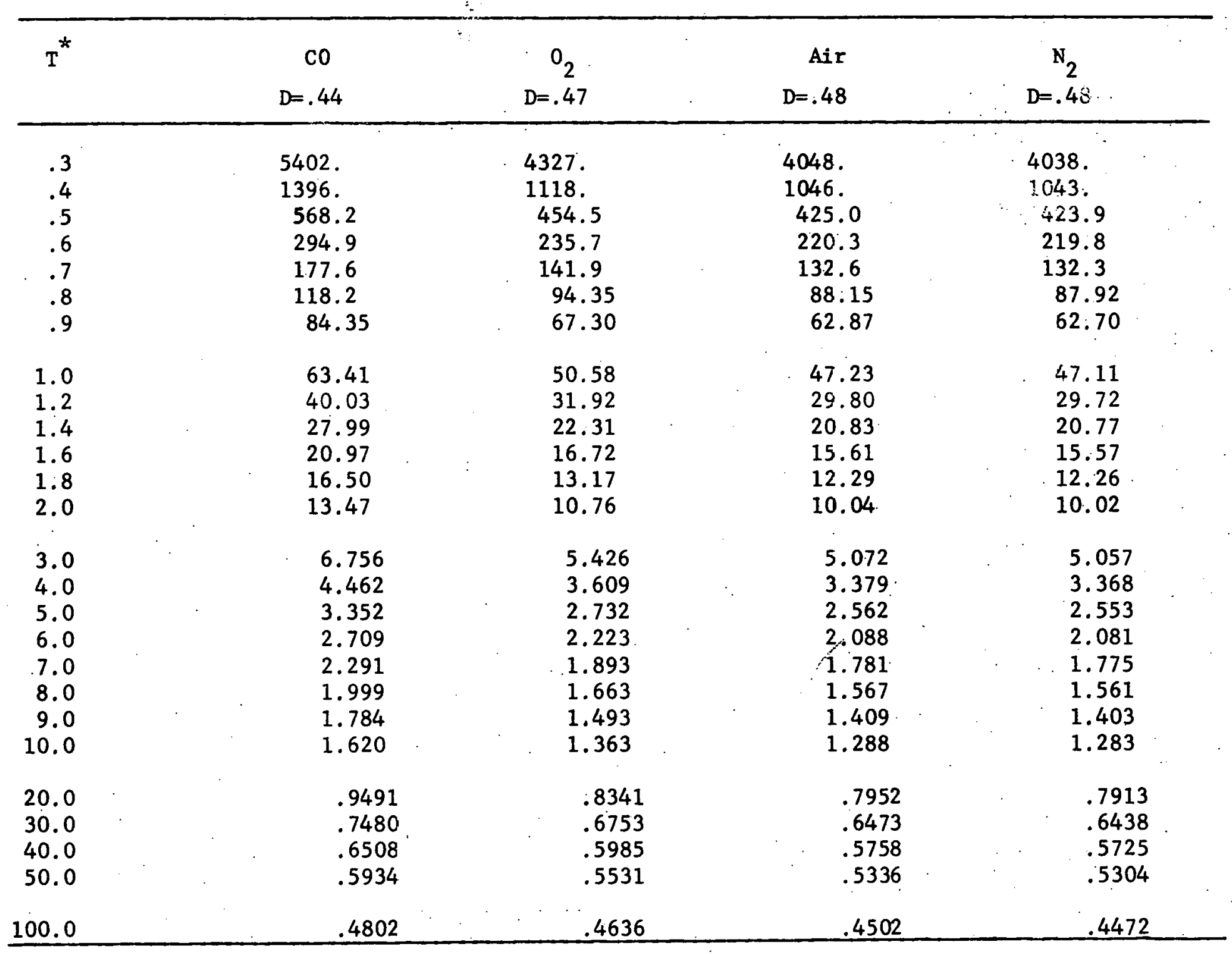


Table 6.4 (Continued)

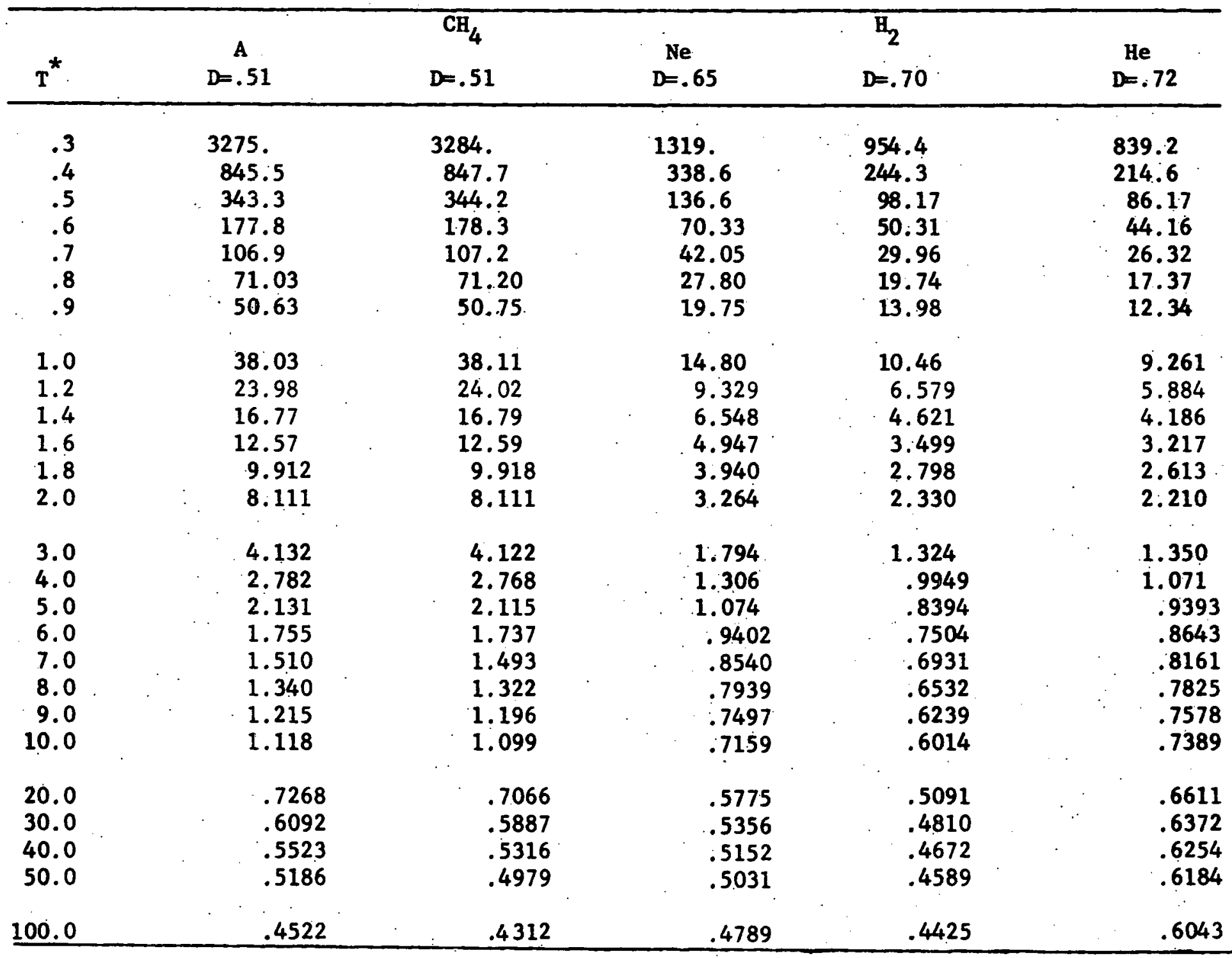

$\overrightarrow{0}$ 


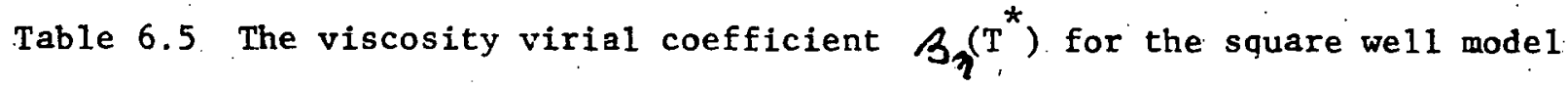

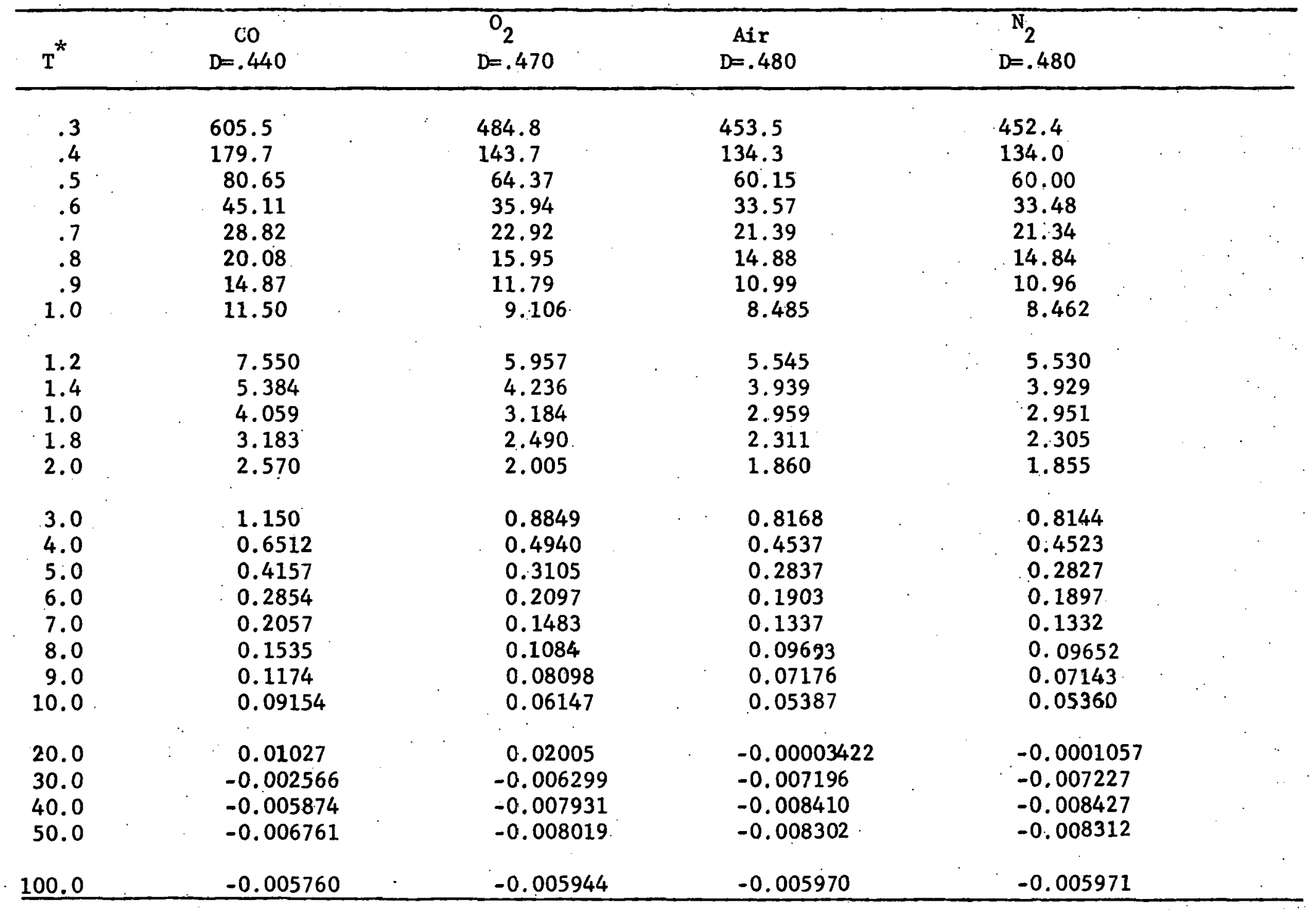


Table 6.5 (Continued)

\begin{tabular}{|c|c|c|c|c|c|c|}
\hline $\mathrm{T}^{*}$ & $\begin{array}{c}\mathrm{Ar} \\
\mathrm{D}=.510\end{array}$ & $\begin{array}{c}\mathrm{CH}_{4} \\
\mathrm{D}=.510\end{array}$ & $\begin{array}{c}\mathrm{Ne} \\
\mathrm{D}=.650\end{array}$ & $\begin{array}{c}\mathrm{H}_{2} \\
\mathrm{D}=.700\end{array}$ & $\begin{array}{l}\mathrm{He} \\
\mathrm{D}=.720\end{array}$ & \\
\hline $\begin{array}{l}.3 \\
.4 \\
.5 \\
.6 \\
.7 \\
.8 \\
.9 \\
1.0 \\
1.2 \\
1.4 \\
1.6 \\
1.8 \\
2.0\end{array}$ & $\begin{array}{c}366.8 \\
108.5 \\
48.49 \\
27.00 \\
17.18 \\
11.92 \\
8.790 \\
6.775 \\
4.413 \\
3.124 \\
2.339 \\
1.821 \\
1.461\end{array}$ & $\begin{array}{c}367.7 \\
108.7 \\
48.61 \\
27.08 \\
17.22 \\
11.95 \\
8.814 \\
6.793 \\
4.425 \\
3.133 \\
2.346 \\
1.827 \\
1.465\end{array}$ & $\begin{array}{c}147.8 \\
43.43 \\
19.23 \\
10.60 \\
6.667 \\
4.577 \\
3.341 \\
2.549 \\
1.626 \\
1.128 \\
0.8274 \\
0.6318 \\
0.4963\end{array}$ & $\begin{array}{c}107.3 \\
31.49 \\
13.91 \\
7.630 \\
4.783 \\
3.269 \\
2.372 \\
1.799 \\
1.140 \\
0.7837 \\
0.5688 \\
0.4290 \\
0.3330\end{array}$ & $\begin{array}{c}94.55 \\
27.55 \\
12.24 \\
6.710 \\
4.195 \\
2.864 \\
2.075 \\
1.571 \\
0.9895 \\
0.6787 \\
0.4909 \\
0.3686 \\
0.2844\end{array}$ & . \\
\hline $\begin{array}{r}3.0 \\
4.0 \\
5.0 \\
6.0 \\
7.0 \\
8.0 \\
9.0 \\
10.0\end{array}$ & $\begin{array}{l}0.6309 \\
0.3440 \\
0.2107 \\
0.1380 \\
0.09431 \\
0.06611 \\
0.04699 \\
0.03352\end{array}$ & $\begin{array}{l}0.6329 \\
0.3452 \\
0.2115 \\
0.1386 \\
0.09474 \\
0.06644 \\
0.04726 \\
0.03374\end{array}$ & $\begin{array}{l}0.1900 \\
0.08829 \\
0.04332 \\
0.02006 \\
0.006839 \\
-0.001143 \\
-0.006159 \\
-0.009391\end{array}$ & $\begin{array}{l}0.1193 \\
0.04923 \\
0.01879 \\
0.003573 \\
-0.004745 \\
-0.009550 \\
-0.01240 \\
-0.01409\end{array}$ & $\begin{array}{l}0.09854 \\
0.03818 \\
0.01213 \\
-0.0007835 \\
-0.007678 \\
-0.01157 \\
-0.01380 \\
-0.01506\end{array}$ & 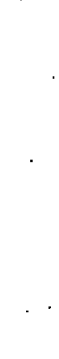 \\
\hline $\begin{array}{l}20.0 \\
30.0 \\
40.0 \\
50.0\end{array}$ & $\begin{array}{l}-0.005347 \\
-0.009460 \\
-0.009574 \\
-0.008955\end{array}$ & $\begin{array}{l}-0.005292 \\
-0.009437 \\
-0.009563 \\
-0.008949\end{array}$ & $\begin{array}{l}-0.01454 \\
-0.01236 \\
-0.01040 \\
-0.008915\end{array}$ & $\begin{array}{l}-0.01461 \\
-0.01180 \\
-0.009738 \\
-0.008254\end{array}$ & $\begin{array}{l}-0.01437 \\
-0.01146 \\
-0.009397 \\
-0.007941\end{array}$ & \\
\hline 100.0 & -0.005977 & -0.005978 & -0.005151 & -0.004670 & -0.004466 & \\
\hline
\end{tabular}


Table 6.6 The viscosity virial coefficient $\beta_{q}\left(T^{*}\right)$ for the Lennard-Jones model

\begin{tabular}{|c|c|c|c|c|c|c|c|}
\hline$T^{*}$ & $I_{\eta}^{(1)}$ & $I_{\eta}^{(2)}$ & $\mathrm{I}\left(\begin{array}{c}(3) \\
\eta\end{array}\right.$ & $\sum_{i=1}^{3} I_{\eta}^{(i)}=I_{\eta}$ & 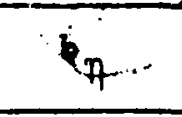 & ${ }^{B} \eta$ & \\
\hline $\begin{array}{l}0.3 \\
0.4 \\
0.5 \\
0.6 \\
0.7 \\
0.8 \\
0.9 \\
1.0\end{array}$ & $\begin{array}{l}-0.8824 \\
-0.8403 \\
-0.8083 \\
-0.7829 \\
-0.7619 \\
-0.7440 \\
-0.7286 \\
-0.7151\end{array}$ & $\begin{array}{l}1.434 \\
0.7277 \\
0.3531 \\
0.1465 \\
0.02867 \\
0.03981 \\
-0.07977 \\
-0.1027\end{array}$ & $\begin{array}{l}-1.652 \\
-1.425 \\
-1.253 \\
-1.116 \\
-1.005 \\
-0.9116 \\
-0.8328 \\
-0.7653\end{array}$ & $\begin{array}{l}-1.100 \\
-1.538 \\
-1.708 \\
-1.752 \\
-1.738 \\
-1.616 \\
-1.641 \\
-1.583\end{array}$ & $\begin{array}{c}40.82 \\
13.80 \\
6.872 \\
4.231 \\
2.972 \\
2.279 \\
1.858 \\
1.583\end{array}$ & $\begin{array}{l}39.72 \\
12.26 \\
6.701 \\
2.479 \\
1.234 \\
0.5840 \\
0.2170 \\
0.0000\end{array}$ & \\
\hline $\begin{array}{l}1.2 \\
1.4 \\
1.6 \\
1.8 \\
2.0\end{array}$ & $\begin{array}{l}-0.6921 \\
-0.6731 \\
-0.6570 \\
-0.6430 \\
-0.6306\end{array}$ & $\begin{array}{l}-0.1213 \\
-0.1228 \\
-0.1176 \\
-0.1102 \\
-0.1023\end{array}$ & $\begin{array}{l}-0.6563 \\
-0.5731 \\
-0.5082 \\
-0.4565 \\
-0.4144\end{array}$ & $\begin{array}{l}-1.470 \\
-1.369 \\
-1.283 \\
-1.210 \\
-1.147\end{array}$ & $\begin{array}{l}1.258 \\
1.078 \\
0.9669 \\
0.8924 \\
0.8395\end{array}$ & $\begin{array}{l}-0.2120 \\
-0.2910 \\
-0.3161 \\
-0.3176 \\
-0.3075\end{array}$ & \\
\hline $\begin{array}{r}3.0 \\
4.0 \\
5.0 \\
6.0 \\
7.0 \\
8.0 \\
9.0 \\
10.0\end{array}$ & $\begin{array}{l}-0.5842 \\
-0.5524 \\
-0.5283 \\
-0.5090 \\
-0.4930 \\
-0.4793 \\
-0.4674 \\
-0.4569\end{array}$ & $\begin{array}{l}-0.07037 \\
-0.05169 \\
-0.04037 \\
-0.03292 \\
-0.02764 \\
-0.02373 \\
-0.02077 \\
-0.01849\end{array}$ & $\begin{array}{l}-0.2819 \\
-0.2108 \\
-0.1678 \\
-0.1400 \\
-0.1209 \\
-0.1067 \\
-0.09551 \\
-0.08635\end{array}$ & $\begin{array}{l}-0.9365 \\
-0.8149 \\
-0.7365 \\
-0.6819 \\
-0.6415 \\
-0.6097 \\
-0.5837 \\
-0.5617\end{array}$ & $\begin{array}{l}0.7077 \\
0.6508 \\
0.6165 \\
0.5922 \\
0.5733 \\
0.5579 \\
0.5448 \\
0.5334\end{array}$ & $\begin{array}{l}-0.2595 \\
-0.1641 \\
-0.1200 \\
-0.08970 \\
-0.06820 \\
-0.05180 \\
-0.03890 \\
-0.02830\end{array}$ & \\
\hline $\begin{array}{l}20.0 \\
30.0 \\
40.0 \\
50.0\end{array}$ & $\begin{array}{l}-0.3913 \\
-0.3562 \\
-0.3327 \\
-0.3154\end{array}$ & $\begin{array}{l}-0.009079 \\
-0.006126 \\
-0.004763 \\
-0.003805\end{array}$ & $\begin{array}{l}-0.04104 \\
-0.02678 \\
-0.02094 \\
-0.01766\end{array}$ & $\begin{array}{l}-0.4414 \\
-0.3891 \\
-0.3584 \\
-0.3369\end{array}$ & $\begin{array}{l}0.4641 \\
0.4268 \\
0.4015 \\
0.3826\end{array}$ & $\begin{array}{l}0.02270 \\
0.03770 \\
0.04310 \\
0.04570\end{array}$ & \\
\hline 100.0 & -0.2664 & -0.001633 & -0.009006 & -0.2770 & 0.3280 & 0.05100 & \\
\hline
\end{tabular}


Table 6.7 The thermal conductivity virial coefficient $\beta_{\lambda}\left(T^{*}\right)$ for the Lennard-Jones model

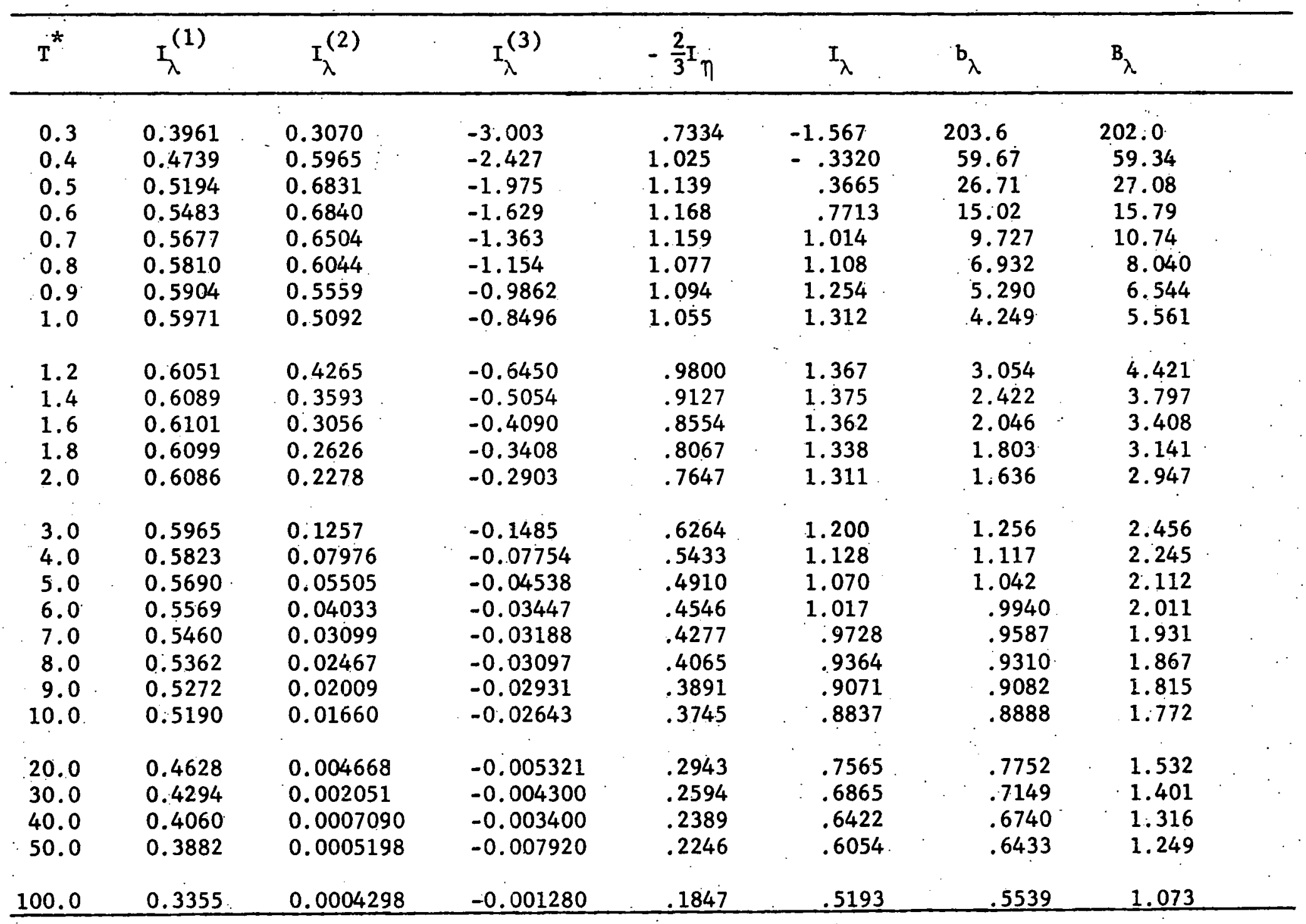




\section{LITERATURE CITED}

1. J. G. Kirkwood, J. Chem. Phys. 14, 180 (1946).

2. L. Bo'ltzmann, Lectures on Gas Theory (University of Caj.ifornia Press, Berkeley and Los Angeles, 1964).

3. S. Chapman and T. G. Cowling, The Mathematical Theory of Non-Uniform Gases (Cambridge University Press, Naw York, 1953).

4. J. DeBoer, Rept. Progr. Phys. 12, 305 (1949).

5. J. W. Gibbs, Elementary Principles in Statistical Mechanics (Dover Publications, Inc., New York, 1960).

6. J. G. Kirkwood, J. Chem. Phys. 15, 72 (1947).

7. M. Born and H. S. Green, A General Kinetic Theory of

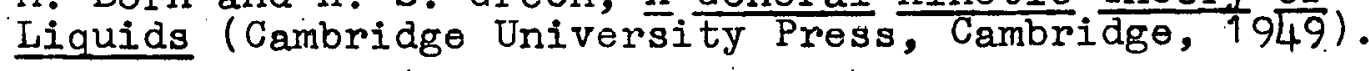

8. J. H. Jeans, Kinetic Theory of Gases (Cambridge University Press, Cambridge, 1925$)$.

9. N. N. Bogoliubov, Studies in Statistical Mechanics (North-Holland Publishing Co., Amsterdam, 1961), Vol. I.

10. G. E. Uhlenbeck and G. W. Ford, Fundamental Problems in Statistical Mechanics (American Mathematical Society, Providence, Rhode Island, 1963).

11. M. H. Ernst, L. K. Haines and J. R. Dorfman, Rev. Mod. Phys. 41, 296 (1969).

12. E. G. D. Cohen, in Statistical Mechanics at the Turn of the Dooade, E. C. D. Oohen, Ed., (Marcel Dekker, Inc., New York, 1971).

13. J. Yvon, La Theorie Statistiques des Fluids (Actualites Scientifiques et Industrielles, Hermann, Paris, 1935).

14. S. T. Choh and G. E. Uhlenbeck, The Kinetic Theory of Dense Gases (Univ. of Michigan Report, 1958).

15. J. R. Dorfman and E. G. D. Cohen, J. Math. Phys. 1,282 (1966).

16. E'. A. Frieman and R. Goldman, Bull. Am. Phys. Soc. 10, 531 (1965). 
17. K. Kawasaki and I. Oppenheim, Phys. Rev. 139, A 1763 $(1965)$.

18. H. J. M. Hanley, R. D. McCarty and J. V. Sengers, J. Chem. Phys. 50, 857 (1969).

19. R. F. Snider and C. F. Curtiss, Phys. Fluids 1, 122 (1958).

20. R. F. Snider and C. F, Curtiss, Phys, Fluids 3, 903 $(1960)$.

21. D. K. Hoffman and C. F. Curtiss, Phys. Fluids I, 1887 (1964).

22. D. K. Hoffman and C. F. Curtiss, Phys. Fluids $\underline{8}, 667$ (1965).

23. D. K. Hoffman and C. F. Curtiss, Phys. Fiuids 8,890 (1965).

24. R. Courant and D. Hilbert, Methods of Mathematical Physics (Interscience Pubiishers, Inc., New York, 1953).

25. H. S. Green and D. K. Hoffman; J. Chem. Phys. 49, 2600 (1968).

26. H. S. Green, in Kinetic Equations, R. L. Liboff and N. Rostoker, Eds., Gordon and Breach, Now York, 1971).

27. D. Burmett, Proc. Lond. Math. Soc. 39, 385 (1935).

28. R. B. Bird, W. E. Steward and E. N. Lightfoot, Transport Phenomena (J. Wiley and Son, New York, 1965).

29. J. W. Gibbs, Vector Analysis (Dover Publications, Inc., New York, 1960 ).

30. 3, Chapter 16

31. K. F. Snider and F. R. PicCourt, Phys. Fluids 6, 1020 (1963).

32. 3, Chapter 7

33. J. O. Hirshfelder, C. F. Curtiss and R. B. Bird, Molecular Theory of Gases and Liquids ( $\mathrm{J}$. Wiley and Sons, Inc., New York, 1954).

34. J. H. Irving and J. G. Kirkwood, J. Chem. Phys. 18, 817 (1950). 
35. H. Goldstein, Classical Mechanics (Addison-Wesley Publishing Company, Inc., Reading, Mass., 1950).

36. D. E. Stogryn and.J. O. Hirshfelder, J. Chem. Phys. 31 , 1531 (1959).

37. M. Strasburger and M. L. Occell1, Computational Aspects of Multiple Integral Evaluation in the Kinetic Theory of a Moderately Dense Lennard-Jones Gas (In Preparation).

38. C. F. Curtiss, M. B. Mciliroy and D. K. Hoffman, Int. J. Engng. Sc1. 3, 269 (1965).

39. D. K. Hoffman, Ph. D. Dissertation, University of Wisconsin, Madison, 1964.

40. C. E. Froberg, Introduction to Numerical Analysis (Addison-Wesley Publishing Company, Inc., Reading, Mass. 1965):

41. IBM Corp., System/360 Scientific Subroutine Package, 360A-CM-03X, Version III Programer's Manual (IBM Corp., White Plains, New York, 1968).

42. E. M. Holleran and H. M. Hulburt, J. Chem. Phys. 19, 233 (1951).

43. G. P. Flynn, R. V. Hanks, N. A. Lemaire and J. Ross, J. Chem. Phys. 38, 154.(1963).

44. J. V. Sengers, W. T. Bolk and C. J. Stigter, Physica 30, $1018(1964)$. 


\section{ACKNOWLEDGMEN'S}

The author wishes to thank the "American taxpayer" and in particular the people of Iowa for the following reasons. He came to Ames in 1963 on a Fulbright travel grant and lived during the four years of his undergraduate education in the Delta Tau Delta fraternity whose members provided him with constant friendship and encouragement. Without the help he received from the fraternity, it would have been impossible for him to continue his studies at Iowa State University.

The author also wishes to acknowledge the financial assistance of three Iowa State University scholarships and the teaching and research assistantships provided by the chemistry department.

In addition the author wishes to thank the following persons: Dr. D. K. Hoffman, his major professor, for his teachings and guidance; M. Strasburger for coding the computer program for the calculation of the viscosity virial coefficient of a Lennard-Jones gas, and Prof. R. Lampert for supervising the calculation; his wife Susan for her unlimited. patience and understanding (and most of all, for her typing). 


\section{APPENDIX A}

To prove the result in Eq. (4.2) we use an identity derived by Hoffman and Curtiss (21) to operate on $\underline{v}_{12} \cdot \underline{r}$.

$$
\begin{aligned}
& \underline{v}_{12}^{\prime} \cdot \frac{\partial}{\partial \underline{r}^{\prime}}\left(\underline{v}_{12} \cdot \underline{r}\right)=\underline{v}_{12} \cdot \frac{\partial}{\partial \underline{r}}\left(\underline{v}_{12} \cdot \underline{r}\right)-\frac{2}{m} \frac{\partial}{\partial \underline{r}} \mathscr{R}_{2} \cdot \frac{\partial}{\partial \underline{v}_{12}}\left(\underline{v}_{12} \cdot \underline{r}\right) \\
& \text { - } \underline{v}_{12} \cdot\left(\underline{v}_{12} \cdot \underline{U}\right)-\frac{2}{m} \frac{\partial}{f_{\underline{r}}} q_{2} \cdot \underline{\underline{U}} \cdot \underline{\underline{r}}
\end{aligned}
$$

$$
\begin{aligned}
& \frac{\partial \phi_{12}}{\partial r}=\frac{m}{2 r}\left(v_{12}-\underline{v}_{12}^{\prime} \cdot \frac{\partial}{\partial \underline{r}^{\prime}}\left(\underline{v}_{12} \cdot \underline{r}\right)\right) \\
& \underline{\gamma}=(m / 4 k T)^{\frac{1}{2}} \underline{v}_{12} \\
& \gamma^{2}=\gamma^{\prime 2}-\phi_{12} / k T \\
& \gamma^{\prime 2}=\underline{\gamma}^{\prime} \cdot f_{\underline{r}^{\prime}}\left(\underline{\gamma}^{\prime} \cdot \underline{r}^{\prime}\right)
\end{aligned}
$$

We obtain, upon substitution, the desired expression for $\int_{r} \phi_{12}$ given in $\mathrm{Eq} .(4.2)$. 
10. APPENDIX B

We want to calculate the coefficients $a_{1}, a_{2}, c_{2}, c_{1}$ and $c_{3}$ in Eq. (4.39). From the properties of Dirac's delta f'unction we can se $\theta$ that $w_{c} \neq 0$ only at $z=0, z_{0} / 2, z_{0}$. Let us specifically calculate wo at $z=\epsilon, z_{0} / 2+\epsilon, z_{0}+\epsilon$ and then take the limit as $\epsilon \rightarrow 0$. We find

$$
\text { a. } \begin{aligned}
\int d z \delta(z) & =\int d z\left(\left(\gamma^{\prime} \frac{\gamma}{\partial z}\left(\underline{\gamma}^{\prime} \cdot \underline{r}^{\prime}-\underline{\gamma} \cdot \underline{r}\right)\right)-\phi_{2} / k T\right) \\
& =\left.\gamma^{\prime}\left(\underline{\gamma}^{\prime} \cdot \underline{\underline{r}}^{\prime}-\underline{\gamma} \cdot \underline{\underline{r}}\right)\right|^{\epsilon}+\delta \phi_{12} / \mathrm{k} T
\end{aligned}
$$

After taking the limit as $\epsilon \rightarrow 0$ and using conservation arguments we obtain

$$
\left.a_{1}=\gamma^{\prime}\left(\underline{\gamma}^{\prime} \cdot \underline{c}-\underline{\gamma} \cdot \underline{c}\right)=\gamma^{\prime \prime}\left(c^{2}\left(\gamma^{\prime 2}+\epsilon / k T\right)-\gamma^{\prime 2} b^{2}\right)^{1 / 2}-\gamma^{\prime}\left(c^{2}-b^{2}\right)^{1 / 2}\right)
$$

Using the same arguments employed to derive this expression we find that $a_{1}=a_{2}=c_{1}=c_{3}$. For $c_{2}$ we obtain instead

$$
c_{2} d z \delta\left(z-z_{0} / 2\right)=\left.\gamma^{\prime}\left(\underline{\gamma}^{\prime} \cdot \underline{\underline{r}}^{\prime}-\underline{y} \cdot \underline{r}\right)\right|_{z=\frac{z_{0}}{z}-\epsilon} ^{z=z_{0}+\epsilon}
$$

From which with Eq. $(4.30)$ we find :

$$
c_{2}=-2 \gamma^{\prime}(\underline{\gamma} \cdot \underline{a})=-2 \gamma^{\prime}\left(a^{2}\left(\gamma r^{2}+\epsilon / k T\right)-\gamma^{\prime 2} b^{2}\right)^{\frac{3}{2}}
$$

